\title{
Support for total hip replacement surgery: Structures modeling, Gait Data Analysis and Report system
}

\author{
Gianluca Mario Izzo
}

University of Naples Federico II, Dept. of Biomedical, electronic and telecommunications engineering, Italy; in collaboration with: Dept. of Science, Education and Innovation, Landspitali University Hospital, Iceland and Dept. of Biomedical Engineering, University of Reykjavik, Iceland

\begin{abstract}
For the treatment of advanced damages of hip joints, Total Hip Arthroplasty is well proven. Due to the different mechanical properties of the prosthesis material and the bone tissue, a partial unloading of the periprosthetic bone occurs. The bone cement causes reduction in bone density as a result of removal of normal stress from the bone, leading to weakening of the bone in that area and the fracture risk increases. Bone loss is identified as one of the main reasons for loosening of the stem. Otherwise, thanks to the press-fit of the non-cemented stem achieved by surgery, the bone layers immediately adjacent to the stem are preloaded, thus encouraged growing, and the bone getting stronger. The non-cemented stem would be the better choice for every patient, but the question remains if the femur can handle the pressfitting surgery. This studies aim to develop a monitoring techniques based on Gait analysis and bone density changes to assess patient recovery after Total Hip Arthroplasty. Furthermore, to validate computational processes based on 3D modeling and Finite Element Methods for optimizing decision making in the operation process and selecting the suited surgical procedure. A vision could be minimizing risk of periprosthetic fracture during and after surgery. Patients: The sample presents 11 patients receiving cemented implant and 13 for the uncemented. Patients are grouped by type of implant. Three checkpoints were considered: before, after operation and one year later. CT scans, gaitrite and kinepro measurements have been realized. Main outcome measures: Fracture risk probability is higher in bone with low bone mineral density; therefore bones are more fragile in elderly people. BMD is indeed one parameter considered among all the observations. Periprosthetic fracture of the femur is a rare but complex complication of THA, and requires demanding surgery. As such, they result in considerable morbidity and dysfunction. Thus, tests of force reaction have been accomplished to support surgeons during the prosthesis fitting. Identification of risk factors for fracture will improve preoperative counseling and aid primary prevention. To assess eventual improvements or find out trends respecting the implant used, gait data have been collected and compared with muscles mass modeling.
\end{abstract}

Key Words: Total Hip Replacement, Bone Mineral Density, Gait Analysis, 3D Modeling, Surface electromyography

European Journal Translational Myology - Basic Applied Myology 2012; 22(1\&2): 69-121

1 Introduction

1.1 Context

1.2 Workflow

1.3 Analysis Platforms

1.4 Patients: the sample

2 Background: Bones and Hip Joint

2.1 Bones
2.1.1 Anatomy of a bone

2.1.2 Histology of bone tissue

2.1.3 Formation, growth and replacement

2.2 Aging and bone tissue

2.3 Hip (Coxal) joint

2.3.1 Anatomy of the joint

2.3.2 Movements 
Total hip replacement: structures modeling, gait analysis and report

European Journal Translational Myology - Basic Applied Myology 2012; 22 (1\&2): 69-121

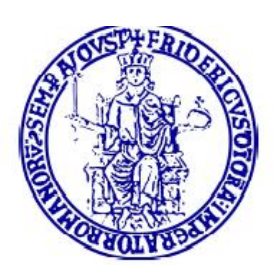

SUPPORT FOR TOTAL HIP REPLACEMENT

SURGERY: STRUCTURES MODELING, GAIT DATA ANALYSIS AND REPORTING SYSTEM

Gianluca Mario lzzo

num. 080000344

Master of Science in Biomedical Engineering

July 2012

School of Engineering Federico II of Naples

in collaboration with

Reykjavik University, Landspitali - University Hospital of Iceland

M.Sc. RESEARCH THESIS

2.3.3 Mechanics in hip joints

3 TUR and implants

3.1 Operation intro

3.1.1 Diseases

3.1.2 Surgical techniques

3.2 Hip joint prosthesis

3.2.1 Intro

3.2.2 Cemented Total Hip Replacement

3.2.3 Cementless Total Hip Replacement

3.2.4 Bone-Implant interface: unsolved problem

3.2.5 The choice

4 Models

4.1 Introduction

4.2 Modeling

4.2.1 Image segmentation

4.2.2 Meshing

4.2.3 Assigning materials properties

4.3 Analyzing

4.3.1 Theory of FEA process

4.3.2 Strain test

4.4 Bone mineral density measurement

4.4.1 Theory of the BMD

4.4.2 Method

4.4.3 Results

5 Gait analyses

5.1 The gait
5.1.1 Phases

5.1.2 Walk parameters

5.1.3 Muscles involved

5.2 Gait rite

5.2.1 Introduction

5.2.2 Method

5.2.3 Results

5.3 sEMG and modeling correlation

5.3.1 sEMG briefing

5.3.2 Setup on muscles

5.3.3 Method

5.3.4 Results

6 Store and report

6.1 Introduction

6.2 DBMS lexis

6.3 Structure and screen shots

7 Conclusions and future perspectives

8 Appendix

\section{INTRODUCTION}

\subsection{Context}

Total hip replacement, i.e. surgical replacement of the hip joint with an artificial prosthesis has been one of the most effective and successful orthopedic interventions for many decades as it reproducibly restores function and reduces pain in formerly pathologic hip joints. THR has been regularly proven to have significant positive impact while being cost effective compared to other procedures. It is applied for several pathologies, mainly in arthrosis, but also as a very beneficial treatment in osteonecrosis of the femoral head and femoral neck fractures. Currently there are two methodological options for THR, cement and cementless implant. Controversy exists regarding the optimal method.

There are no guidelines or clear clinical recommendations currently in practice on the choice of a cemented or an uncemented THR for a specific patient. Many different designs are used both for cemented and uncemented THR where large studies have shown different outcomes differentiating between cemented and uncemented methods. Cemented THR has a higher 10-year survival rate than uncemented THR, i.e. a lower risk of revision (including aseptic loosening. Therefore in summary, the operation to which the patients are subjected compensates functional deficiencies afferent bones (femur and acetabulum), related to genetic or trauma consequences, using a wide variety of prosthesis. Briefly two main options are available: the bone cement used to fix the stem of the new femur causes reduction in bone density as a result of removal of normal stress from the bone. Hypothesized by Wolff's law [48] the bone adapts to the load decrease in 
consequence of stress shielding by resorption. Thus, an aseptic loosening of the implant arises [42]. Bone in a healthy person remodels in response to the load it is placed under, if this load decreases, the bone becomes less dense and weaker for the absence of enough stimuli for continued remodeling required to maintain bone mass. This leads to weakening of the bone in that area and the fracture risk increases, as one of the main reasons for loosening of the stem of our prosthesis. On the other hand, thanks to the press-fit of the noncemented stem achieved by surgery, the bone layers immediately adjacent to the stem are preloaded, thus encouraged to grow, and the bone getting stronger. The uncemented stem would be an adapted choice for each patient, but the question remains if the femur can handle the press-fitting surgery. Moreover, uncemented cup components have a higher risk of cup revision due to aseptic loosening, whereas uncemented stem components have a lower risk of stem revision due to aseptic loosening. Uncemented stems have to be more often revised due to periprosthetic fracture during the first two postoperative years than cemented stems. There is no noticeable difference in risk of infection between the outcomes of cemented compared to uncemented THR. It is apparent that there is no golden standard in THR but the intervention including the various designs and methods has been highly successful and beneficial for many patients [25].

\section{Resources and Targets}

Mainly the efforts of available resources rice toward a support to the work of surgeons, and in case assess subsequent improvements of the patients. Thankfully, this work can be considered as a platform of analysis that beneath the decision making of surgeons. Thus, the "engine" of the work is actually the possibility to take advantage from different experiences in order to reach an adequate level of knowledge useful during all the project phases and to interpret results day by day. The project indeed involves several kinds of knowledge background as the participations of biomedical engineers, surgeons and physiotherapists have been provided. Students from Reykjavik University of Iceland and Federico II University of Naples got an important rule as well. Available facilities are hospital structures, the University of Reykjavik and the Grensas rehabilitation center, a branch of Landspitali specialized in functional recovery of patients. The main project objectives are: Analysis of gait modification before and after the operation, developing a monitoring technique based on Gait analysis in order to get helpful for people involved in rehabilitation assessing any patient recovery after THR. In any clinical analysis, there is an inherent problem of relating the desired outcome to the prior, current, and future states. With muscular-skeletal analysis, this is complicated due to the need for invasive testing methods that may affect the existing condition, fail to give a complete view, or not provide the desired level of detail. For THR, researchers and physicians would prefer to predict the level of compatibility between a given implant geometry and existing bone and muscle structures. The standard tools to perform such structural analysis are Finite Element Analysis (FEA) and 3D modeling. Validate computational processes based on 3D modeling and Finite Element Methods (FEM) for optimizing decision making in THR and selecting the optimal surgical procedure. The use of FEA can minimize risk of periprosthetic fracture during and after surgery by providing evaluation of bone fracture risk based on spiral CT and simulation. Analyze and monitor muscle and bone conditions pre and post THR surgery from medical image, Gait Analysis and sEMG. Evaluation of the synchronization of the activation of the quadriceps muscles in relation to patient's gait phases. Thus, it is persecuted a comparison among pre-and post- operative EMG data, healthy leg and operated leg (also considering the volumes and the shape of the muscles). Evaluation of bone remodeling after THR implant by CT data (possible correlation with gait analysis, foot pressure data, muscle shape, EMG activity). Correlation between micro-CT and CT data for the cancellous and cortical bone, correlating changes in bone turnover markers with changes in BMD after THR with or without cement (Osteoprotegrin, RANKL, Osteocalcin, b-ALP, PINP, CTX-1, NTX-1, DPD).

The project originality can be summarized with following outcomes: Upgraded knowledge concerning bone and muscle conditions pre and post THR observing results provided by different devices for measurements.

Novel methodologies to monitor and correlate different measurements such as: Gait Analysis, EMG, CT scan, micro CT Bone and Muscle density, Bone metabolic activities and Finite element Analysis. Nevertheless each one of them has always been used, together the literature shows a deficiency in studies, thus, the project has been released. As said previously, the project aims to make up an useful tool to estimate fracture risk of the proximal femur bone avoiding problems correlated with fractures during the replacement and in particular the fitting of the implants in femurs. Afterward it will be possible to select more accurately the correct type of hip prosthesis from the beginning. Evaluate whether analysis of bone turnover markers after can be used to predict success of THR.

\subsection{Workflow}

Step by step the going-through-path of the patients enrolled in the program is reported in the following charts. Before the operation patients undergo the CT scan and are subject of gait measurements. After the surgery, roughly six weeks later the operation, new gait measurements are drawn and furthermore one year later as well, with an ulterior CT acquisition. All those actions concerned the relation with patients are 
Total hip replacement: structures modeling, gait analysis and report

European Journal Translational Myology - Basic Applied Myology 2012; 22 (1\&2): 69-121

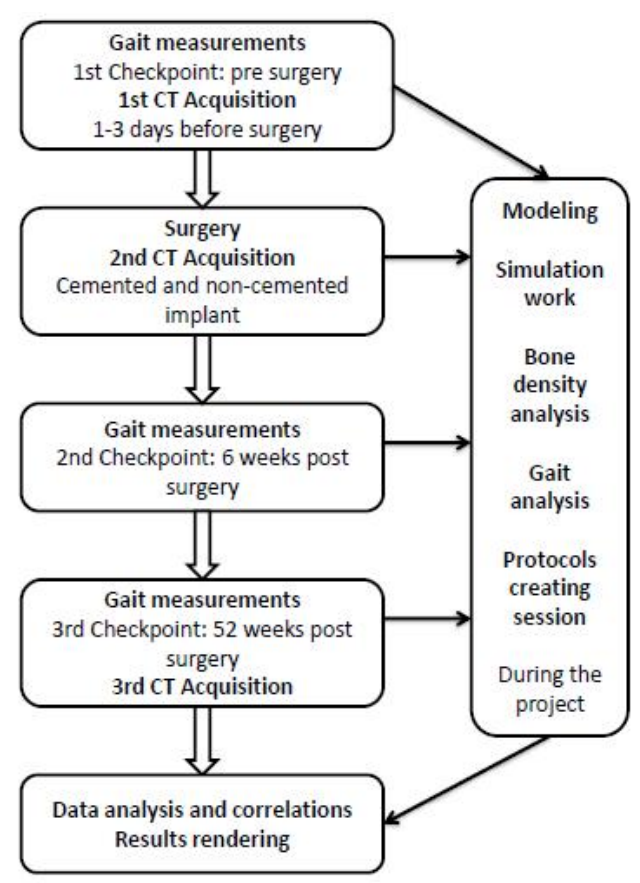

Fig 1 Workflow

continuously surrounded by storing, elaboration, analysis and reporting, as well as shown in the flow chart. The project schedule presents an increasing number of patients, enrolled into a clinical trial.

This group size will provide satisfactory indications to evaluate project feasibility and benefits. To optimize the selection of THR surgical techniques, a standard protocol has been established and improved day by day according to staff observations and needs.

The protocols concern acquisition, elaboration and analysis, creating a real helpful scientific instrument in order to surround surgeon's decisions and foresee possible breaking events on bones structures, furthermore the perspective to assist rehabilitation staff in the post operative phase assessing any improvements by objective evaluations. The work methodology (as the workflow described in Figure 1) applied during the project can be explained in the following way:

- Clinical work: a. Patients will undergo CT scan before and post surgery. The CT protocol starts from the crista and ends at the middle of the femur, slices thickness is $1 \mathrm{~mm}$, slice increment is $0.5 \mathrm{~mm}$ and tube intensity is set to $120 \mathrm{KV}$. This data allow a precise $3 \mathrm{D}$ reconstruction of the regions of interest. A third scan will be taken after 52 weeks from the operation. b. Patients will be measured with Gait analysis technologies at Grensas rehabilitation (Reykjavik) clinic before surgery and again 6 and 52 weeks after surgery. The equipments used in this project are Kine view and GaitRite in order to get the measurements and elaborate them. KineView uses advanced video

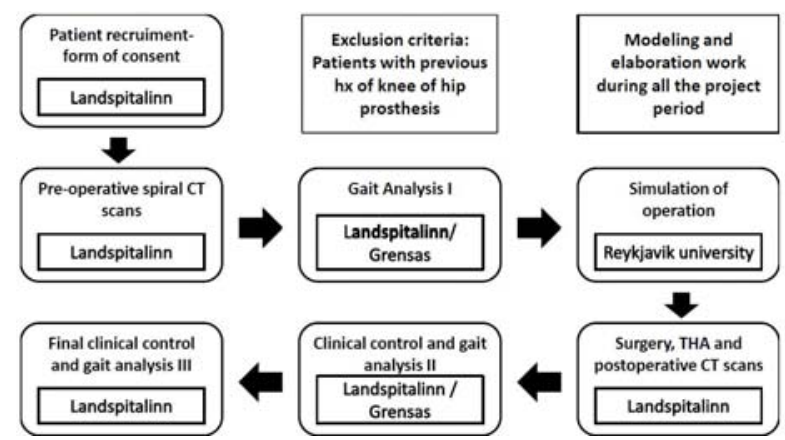

Fig 2 Path

capturing and data processing of patient walk. It is employed in analyzing patient's movement for Parkinson's, Multiple Sclerosis, Stroke, joint replacement, orthopedics. This data will be used to monitor the recovery of each patient, by comparing pre and post surgical acquisitions, and possibly assess present improvements.

- Modeling and simulation: c. The CT images of the femur are imported into a software platform called MIMICS to process and edit 2D image data and perform virtual surgery for both THA techniques. Here 3D models of cement and non-cemented prosthesis are created for each patient. The next step is to divide the $3 \mathrm{D}$ objects into tetrahedral elements, called Meshing. Material properties are then associated to each element of the mesh model (bone, implant stem, metal cup, bone cement and so on) using equations which relate Hounsfield values (from the CT images) to material density. d. The software for the FEM (Ansys Workbench) uses the model developed in c and associates it to materials, applied forces and contact properties. Simulation tools are used in this phase: the strain test evaluates the

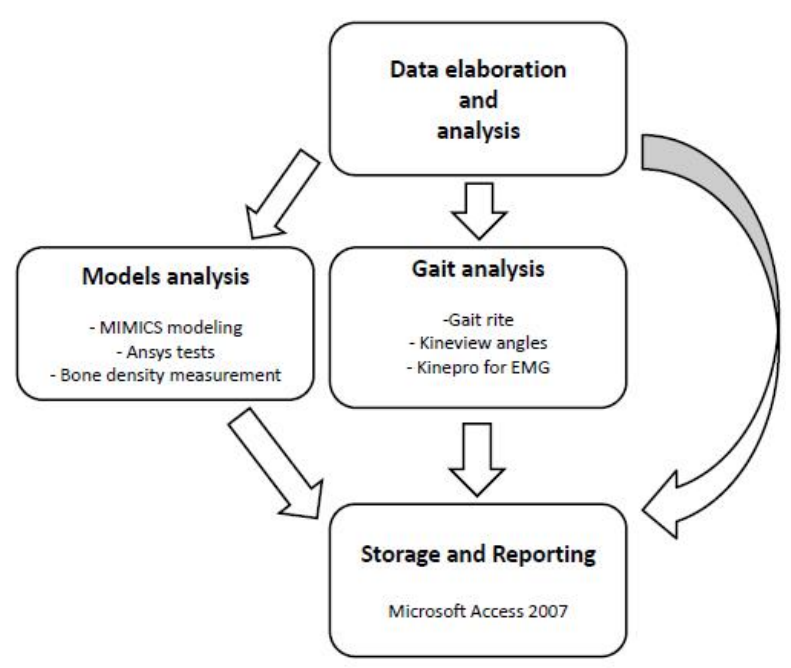

Fig 3 Analysis 
Total hip replacement: structures modeling, gait analysis and report

European Journal Translational Myology - Basic Applied Myology 2012; 22 (1\&2): 69-121

feasibility of the non-cemented surgery. A certain amount of force is applied on the neck of the stem inside the femur, to mimic the press-fit technique used to fix the stem into the femur bone. From equivalent strain the fraction risk is then calculated. This simulation compares the strain distribution in the femur, and a fraction risk is calculated for each type of implant. e. The CT data pre and post surgery will be processed in MIMICS and bone density changes evaluated. f. Evaluation of relationship between features of muscular tissues implicated in leg movement and THA (see Electromyography).

- Data analysis: Fundamentals for the project are check points established in "preoperation", "6 weeks later" and "1 year later" in which all the data are collected, hence these are characterized by the acquisition date and compared each other. The results from all the elaborations will be correlated to the chosen implant type, to patient's anamnesis and subsequent improvements connected with the process of rehabilitation.

\subsection{Analysis Platform}

The phase of data elaboration and assessment surrounds the entire path that the patient follows from the first CT scan acquisition, through measurements, ending with final data analysis and correlations (shown in Figure 2 and 3). The main part of the project regarding acquisition and elaboration is distinguished in two study platforms in which we can theoretically group instruments, data and elaborations: "Model
Table 1 Patients Sample

\begin{tabular}{cccc} 
CEMENTED & & & \\
\hline Age & Gender & Weight & Operated side \\
\hline 71 & F & 95 & Left \\
\hline 76 & M & 86 & Right \\
\hline 73 & F & 80 & Right \\
\hline 66 & F & 66 & Right \\
\hline 71 & F & 91 & Right \\
\hline 58 & M & 74 & Left \\
\hline 68 & F & 81 & Right \\
\hline 66 & F & 75 & Left \\
\hline 77 & F & 80 & Right \\
\hline 67 & F & 80 & Right \\
\hline 75 & F & 63 & Right \\
\hline
\end{tabular}

\begin{tabular}{cccc} 
UNCEMENTED & & & \\
\hline 63 & F & 71 & Right \\
\hline 21 & M & 66 & Right \\
\hline 52 & M & 95 & Left \\
\hline 68 & F & 80 & Right \\
\hline 50 & M & 89 & Right \\
\hline 56 & F & 94 & Left \\
\hline 63 & F & 96 & Left \\
\hline 53 & M & 60 & Left \\
\hline 42 & F & 90 & Right \\
\hline 63 & M & 85 & Left \\
\hline 63 & F & 80 & Right \\
\hline 56 & F & 83 & Right \\
\hline 44 & F & 94 & Left \\
\hline
\end{tabular}
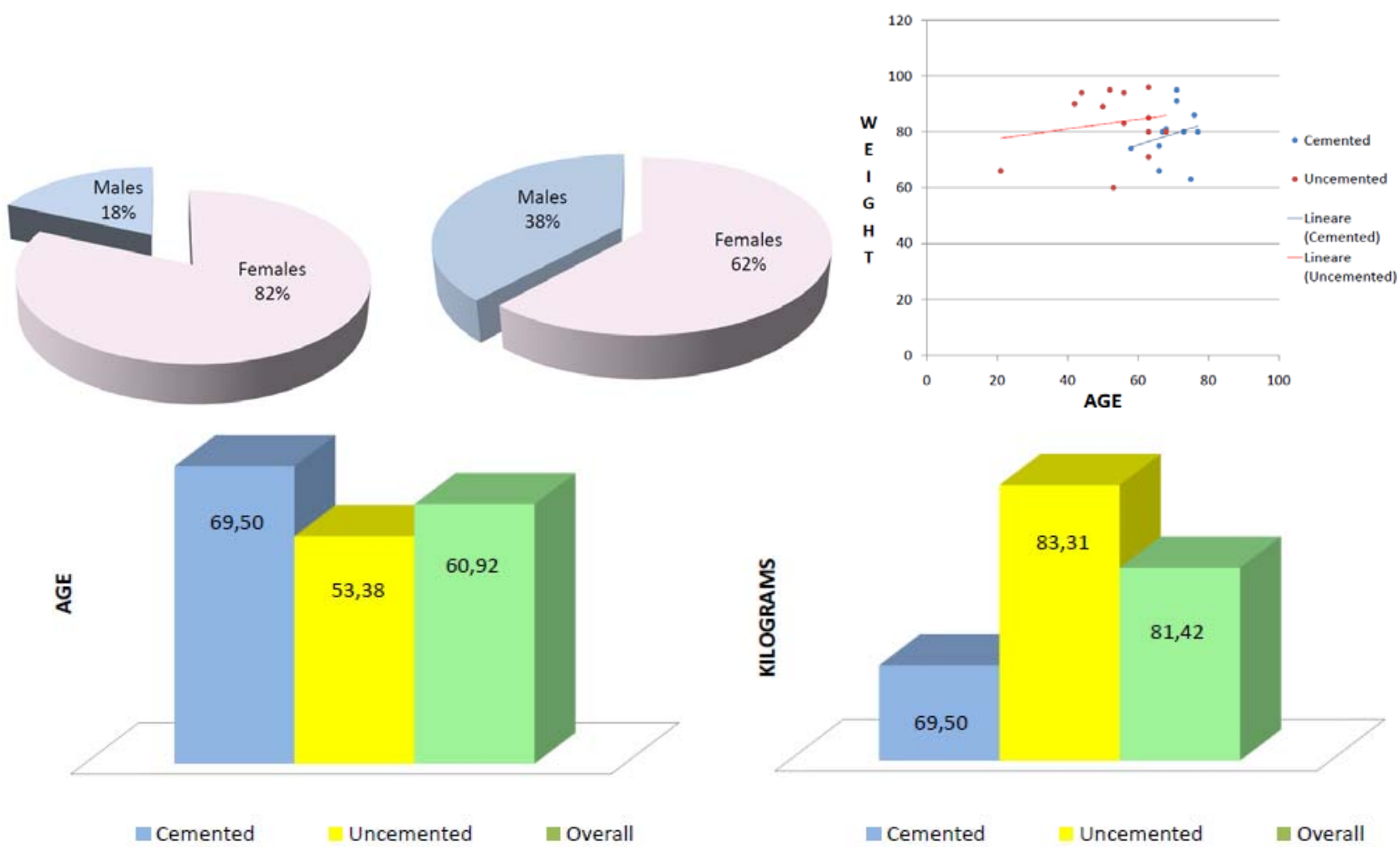

Fig 4 Sample resume 
analysis" and "Gait analysis". The first includes Mimics and Ansys softwares used in order to evaluate mechanical properties of the bones once the implant is fitted under applied forces; 3d models are moreover used to estimate the bone density regarding settled regions of interest on healthy and operated femur. The second part refers to measurements and data reporting in the context of gait analysis, for example angles in the stride or parameters associated to the stride. Meanwhile the results are obtained, it is fundamental for the staff to get an effectively and efficiently information storage aimed to preserve data integrity, to show clear overview understandable by stakeholders. Hence the means is represented by an Access database with which reports are provided. Notice in the figure two different streams already described in which techniques used are classified, observe moreover that not all obtained information need elaboration or anyway are images and signals simply memorized in the storage system. Further details explaining created and complied protocols will be reported in next chapters following the same layout previously given; there will be also argued results, charts and images obtained by the above path and kind of correlations among patients, patients conditions and implant features.

\subsection{Patients: The Sample}

Patients involved have been selected in order to satisfy needs of statistic significant sample: implant type, gender and operation body side are mainly considered; furthermore we obligatorily enrolled patients receiving THR for the first time because of tougher study for particular cases of relapse depending on a wide range of factors. Next tables and charts summarize features of the sample upgraded until the date of writing of this thesis since the number of patients is in continuous increase to achieve reasonable statistic significance and given that there is a major focusing from hospital managers. As well as reported in Table 1, the sample presents 11 patients receiving cemented implant and 13 for the uncemented: besides gender and implant considerations, the interesting aspect is the evident minor age average for CEM patients and the major regarding the weight (Figure 4). Even though will be deepened later, the prospect reveals that it is supposed to use implants with no cement in cases of mild bone degeneration or traumas not in advanced age, instead surgeons would rather apply a cemented implant, still taking into account any peculiarities of each case.

\section{BACKGROUND: BONES AND HIP JOINT}

\subsection{Bones}

Bone tissue is a complex and dynamic living tissue, engages in a continuous process called remodelingbuilding new bone tissue and breaking down old bone tissue. A bone is made up of several different components working together: osseous tissue, cartilage, dense connective tissue, epithelium, various

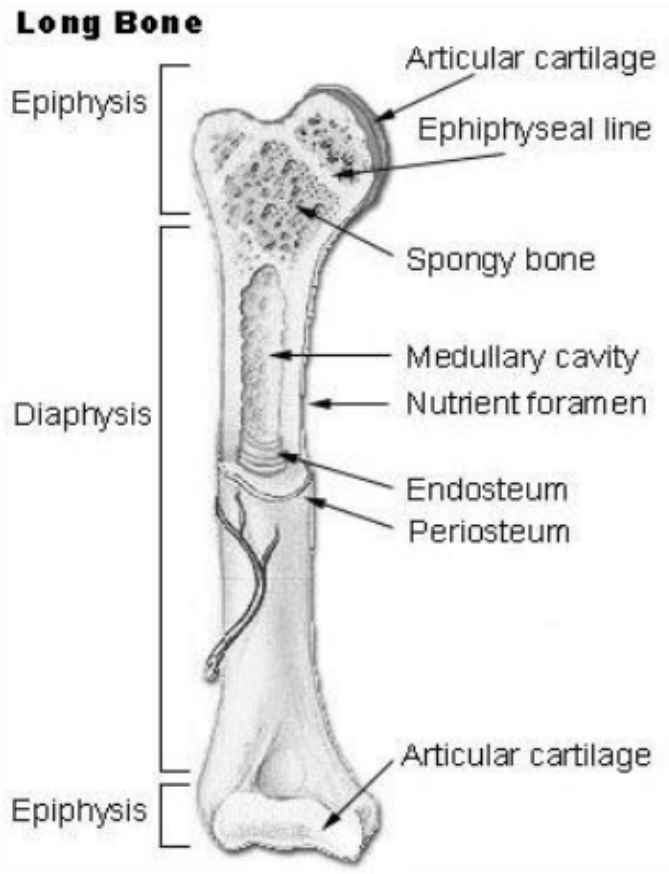

Fig 5 Anatomy of Bones

blood-forming tissues, adipose tissue and nervous tissue. Thus is possible to consider each individual bone as an organ. The entire framework of bones and their cartilage constitute the skeletal system. For the purpose of the thesis this chapter will be focused mainly on hip joint bones reporting briefly bone histology and tissues features.

\subsubsection{Anatomy of bones}

The structure of a bone may be analyzed by considering the parts of a long bone such as the

Humerus (the arm bone) or the femur (the thigh bone). In the human body there are different kinds of bones as long, short, flat or irregulars but because of the thesis purpose we will focus on long bone that have greater length than width and typically consists of the following parts described and as depicted in Figure 5:

- Diaphysis is the bone's shaft, or body, the long cylindrical main portion of the bone.

- Epiphyses are the distal and proximal ends of the bone.

- Metaphyses are the regions in a mature bone where the diaphysis joins the epiphyses. In a growing bone, the metaphyses are regions that include the epiphyseal plate, the point at which cartilage is replaced by bone. The epiphyseal plate is a layer of hyaline cartilage that allows the diaphysis of the bone to grow in length, but not in width. When bone growth in length stops, the cartilage in the epiphyseal plate is replaced by bone and the resulting bony structure is known as the epiphyseal line. Articular cartilage is a thin layer of hyaline cartilage that covers each epiphysis where the bone forms an 
articulation with another bone. Articular cartilage reduces friction and absorbs shock at freely movable joints. Periosteum is a though sheath of dense surface wherever it is not covered by articular cartilage. The periosteum contains bone-forming cells that enable bone to grow in width, but not in length. It also protects the bone, assists in fracture repair, helps nourish bone tissue, and serves as an attachment point for ligaments and tendons. Medullary cavity is the space within the diaphysis that contains fatty yellow bone marrow in adults. Endosteum is a thin membrane that contains bone-forming cells and lines the medullary cavity.

\subsubsection{Histology of Bone Tissues}

Bone consists of $65 \%$ mineral, $35 \%$ organic matrix, cells and water. The bone mineral, in form of small crystals, is placed between collagen fibres. The mineral is largely impure hydroxyapatite, $\mathrm{Ca}_{6}\left(\mathrm{PO}_{4}\right)_{6}(\mathrm{OH})_{2}$, containing carbonate, citrate, fluoride and strontium. The organic matrix consists of $90 \%$ collagen and about $10 \%$ non collagenous proteins. From a mechanical point of view, the bone matrix is comparable to a composite material: the organic matrix is responsible to give toughness to the bone, while the inorganic matrix has the function to stiffen and strengthen the bone [16]. Cells of the tissue bone is permeated by and Support for total hip replacement surgery lined by various kinds of specialized cells, which here are listed and briefly described in their properties. Osteoprogenic cells have the capacity of mitosis and further differentiation and specialization into mature bone cell. They could be divided in two sub-groups and are the only bone cells to undergo cell division: on type gives rise to bone forming osteoblasts, the other type gives rise to bone resorbing osteoclasts. Both types are commonly found near bone surface as periosteum, endosteum and in canals containing blood vessels [47]. Bone-lining cells cover all surfaces of bones, including the blood channels, forming a thin continuous sheet that controls the movement of ions between the body and the bone. The layer of cells on the outside of the bone is called periosteum while the layer inside is endosteum. These cells usually are considered quiescent osteoblasts deriving via complex series of changes from osteoprogenitor cells. Osteoblasts are bone-forming cells that synthesize and secrete unmineralized bone matrix (the osteoid, Figure 6). They seem to participate in the calcification and resorption of bone and to regulate the flux of calcium and phosphate in and out of bone. Osteoblasts occur as a layer of contiguous cells which in their active state are cuboidal (15 to 30 micron thick). Bone formation occurs in two stages: matrix formation followed by mineralization, denoted by deposition of crystals of hydroxyapatite. Their life cycle can be summarized as follows: birth from a progenitor cell, differentiation from stem cells to osteoblasts and participation in elaborating matrix calcifying units and return to the pre-osteoblast pool, transform into bone-lining cell and burial as osteocytes, or death. The development of osteoblasts and osteoclasts are linked on a molecular basis. Both are derived from precursor cells originating in bone marrow, and osteoblast Differentiation is a prerequisite for osteoclast development. Osteoclasts are bone-resorbing cells, which contain one to more than 50 nuclei and range in diameter from 20 to over 100 Îijm. Their role is to resorb bone, by solubilising both the mineral and the organic component of the matrix. The signals for the selection of sites to be reabsorbed are unknown. Biphoshponates, calcitonin and estrogen are commonly used to inhibit resorption. These are believed to act by
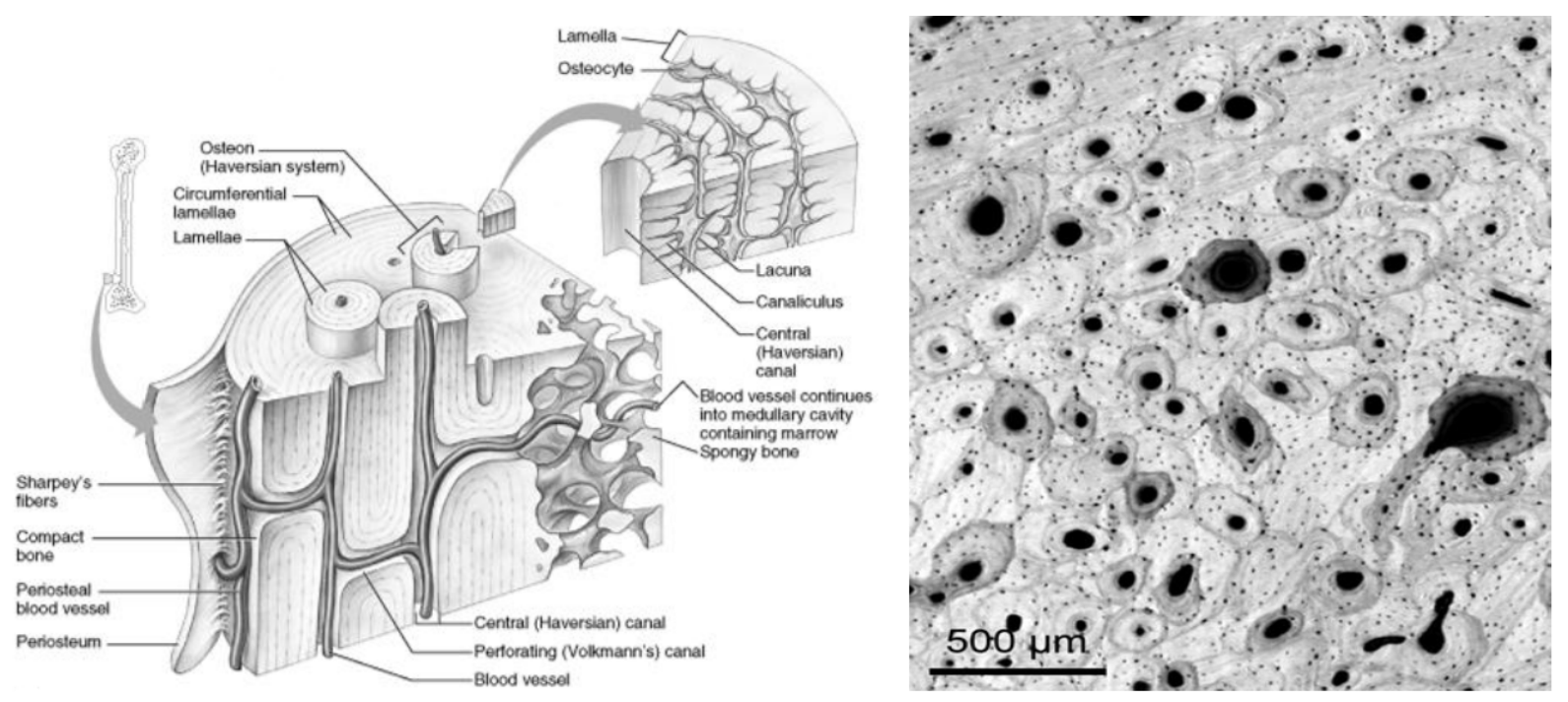

Fig 6 Harvesian System 
inhibiting the formation and activity of osteoclats and promoting osteoclasts apoptosis. Osteocytes are cells in the body of the bone deriving from osteoblasts, imprisoned in the hard bone tissue and connect with neighboring osteocytes and with bone lining cells by means of processes housed in little channels (canaliculi).The osteocytes are thought to be the cells best placed to sense the magnitude and distribution of strains. They are thought both to respond to changes in mechanical strain and to disseminate fluid flow to transduce information to surface cells, via the canalicular processes and the communicating gap junctions. Osteocytes play a key role in homeostatic, morphogenetic and restructuring process of bone mass that constitute the regulation of mineral and architecture. Tissue type: Compact and spongy structures Bone in human and other mammal bodies is generally classified into two types cortical bone, also known as compact bone and trabecular bone, also known as cancellous or spongy bone. These two types are classified as on the basis of porosity and the unit microstructure. Cortical bone is much denser with a porosity ranging between $5 \%$ and $10 \%$, is found primary in the shaft of long bones and forms the outer shell around cancellous bone at the end of joints and the vertebrae. A microscope example has been given in Figure 7 where graphically it is evidenced the porosity difference which mirrors in mechanical different properties. Compact bone tissue is arranged in units called Haversian systems or osteons. A typical osteon is a cylinder about 200 micron in diameter, consisting of a central canal (Haversian canal) surrounded by about 20-30 concentric lamellae, the corresponding circumference of the shaft of long bones is surrounded by several layers of lamellae, immediately underneath the periosteum and on the internal surface adjacent to the endosteum. These lamellae are called circumferential lamellae. In the gaps between Haversian systems can be found interstitial lamellae, as angular fragments of previous concentric and circumferential lamellae. Within the Haversian canals run blood vessels, lymphatics, nerves. The Haversian canals are interconnected by transverse canals, also called the Volkmann canals, which also allow the communication with the periosteum and bone marrow. The outer border of each osteon is surrounded by a cement line, which is a 1 - to 2 micron-thick layer of mineralized matrix, deficient in collagen fibres. Throughout the bone, small cavities (lacunae) containing entrapped bone cells (osteocytes) are found. Microscopic tubular canals (canaliculi) connect the lacunae to each other and to the Haversian canal. The structural unit of trabecular bone is the trabecular packet, a hemiosteon. Ideally, it is shaped like a shallow crescent with a radius of 600 micron, 50 micron thick and $1 \mathrm{~mm}$ long. As with cortical bone, cement lines hold the trabecular packets together. Spongy bone tissue is in contrast with the compact one, does not contain osteons and consists of lamellae that are arranged in an irregular lattice of thin columns of bone called trabeculae. The macroscopic spaces between these are filled with red bone marrow in some bones, which produces blood cells. Within each trabecula are osteocytes that lie in lacunae. Radiating from the lacunae are canaliculi; osteocytes in the trabeculae receive nourishment directly from the blood circulating through the medullary cavities. This tissue tends to be located where bones are not heavily stressed or where stresses are applied from many directions. Spongy bone tissue is lighter, which reduces the overall weight of a bone so that it moves more ready when pulled by a skeletal muscle; furthermore, the trabeculae of spongy bone tissue support and protect the red bone marrow.

\subsubsection{Formation, growth and replacement}

The bone metabolism is one of the processes, which the body uses in order to regulate the homeostasis, thus the bone conformation has to be continuously adapted to morphological and functional needs.

\section{Bone formation}

The process by which bone forms is called ossification or osteogenesis. The skeleton of a human embryo is composed of fibrous connective tissue membranes formed by condensed embryonic connective tissues (mesenchyme) or pieces of hyaline cartilage that resemble the shape of bones. These embryonic tissues provide the template for subsequent ossification, which begins during the sixth or seventh week of the development and follows one of two patterns. This two kinds of ossifications lead to differences in the structure of mature bones; they are simply different methods for bones formation. Intramembranous ossification is the formation of bone directly on or within fibrous connective tissue membranes formed by condensed mesenchyme without first going throughout a cartilage stage. Endochondral ossification is the formation of bone within hyaline cartilage, is a process in which mesenchymal cells are transformed into chondroblasts. These initially produce a hyaline

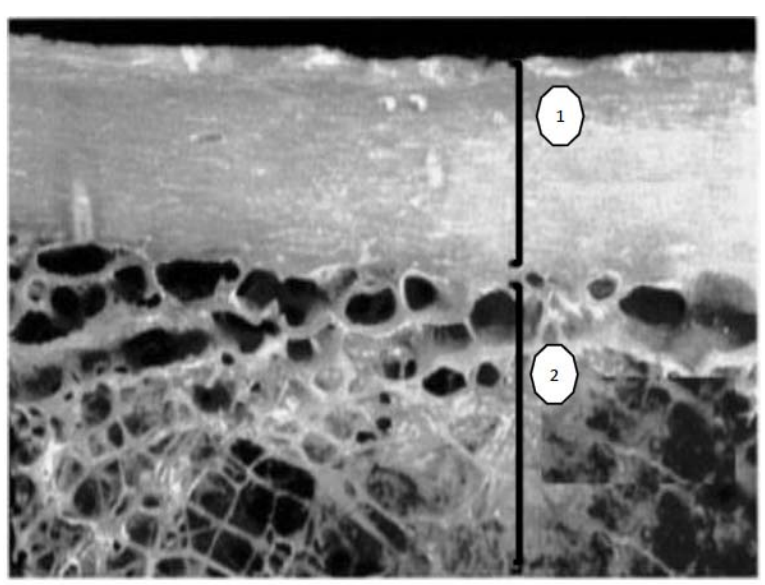

Fig 7 Trabecular and Sponge Bone 
cartilage "model" of the bone. Subsequently, osteoblasts gradually replace the cartilage with bone. Bone growth: By the activity of the epiphyseal plate [43] the diaphysis increases the length. As a bone grows, chondrocytes proliferate on the epiphyseal side of the plate and news of these cover older ones, which are then destroyed by calcification. Thus, the cartilage is replaced by bone on the diaphyseal side of the plate. Therefore the thickness of the epiphyseal plate remains relatively constant, but the bone on the diaphyseal side increases in length. Between 18 and 25 years the plate closes and the process does not go ahead in length growing. The growth in width of the bones is quite different and omitted in this part.

\section{Bone replacement}

Bone, like the skin, forms before birth but continually renews itself thereafter. Remodeling is the ongoing replacement of old bone tissue by new one. Bone constantly remodels and redistributes its matrix along lines of mechanical stress. Compact bone is formed from spongy bone. However, even after bones have reached their adult shapes and sizes, old bone is continually destroyed and new bone tissue is formed in its place. Remodeling also removes worn and injured bone to serve as the body's reservoir for calcium. Several hormones continually regulate exchanges of calcium between blood and bones. The modeling is in general linked with growth, allows the development of normal architecture during growth, controlling the shape, size, strength and anatomy of bones and joints. It increases the outside cortex and marrow cavity diameters, gives shape to the ends of long bones, drifts trabeculae and cortices, enlarges the cranial vault and changes the cranial curvature. During normal growth, periostal bone is added faster by formation drifts than endosteal bone is removed by resorption drifts. This process is regulated so that the cylindrical shaft markedly expands in diameter, whereas the thickness of the wall and the marrow cavity slowly increase. Modeling controls also the modulation of the bone architecture and mass when the mechanical condition changes [18]. For example, bone surfaces can be moved to respond to mechanical requirements. A coordinate action of bone resorption and formation of one side of the periosteal and endosteal surfaces can move the entire shaft to the right or left, allowing some bones to grow eccentrically. Bone resorption is a process involving the breakdown of bone by specialized cells known as osteoclasts. It occurs on a continual level inside the body, with the broken down bone being replaced by new bone growth. As people age, the rate of resorption tends to exceed the rate of replacement, leading to conditions like osteoporosis. In addition, certain medical conditions such as hormone imbalances can cause bone resorption to increase, leading to increased susceptibility to fractures. Despite the actual mechanism for the activation of osteoclasts bone resorption is still unclear, it is known that the process takes place where osteoclasts come in contact with the surface of bone, these begin to erode the bone forming cavities (lacunae of Howship) in cancellous bone, and forming cutting cones or resorption cavities in cortical bone. The resorption process occurs in two simultaneous steps which are dissolution of mineral and enzymatic digestion of organic macromolecules (Figure 8).

\subsubsection{Aging and bone tissue}

In young adults the rates of bone deposition and resorption are about the same; as the level of sex steroids diminishes during middle age, a decrease in bone occurs because bone resorption occurs more rapidly than bone gain. Because women's bones generally are smaller and less massive than men's bones to begin with, loss of bone mass in old age typically has a greater adverse effect in women. There are two different principal effects of aging: loss of bone mass and brittleness. The first effect results from the loss of calcium and other minerals from bone matrix (demineralization). This loss usually begins after age 30 in females, accelerates greatly around 45

\section{Bone Remodeling}
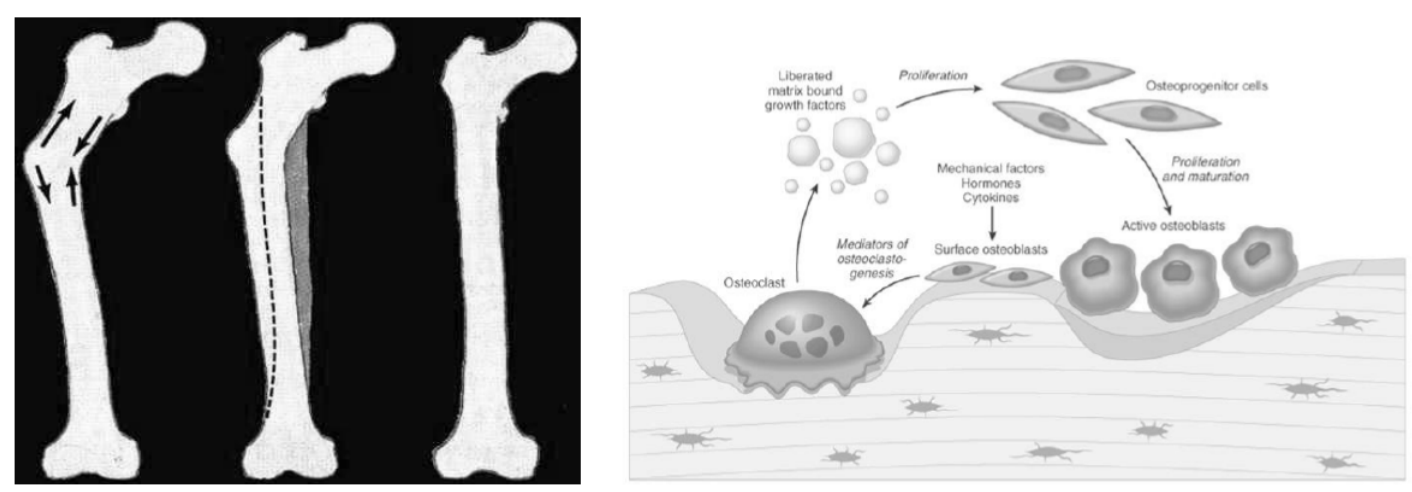

Fig 8 Trauma Deformation and Cycle process 


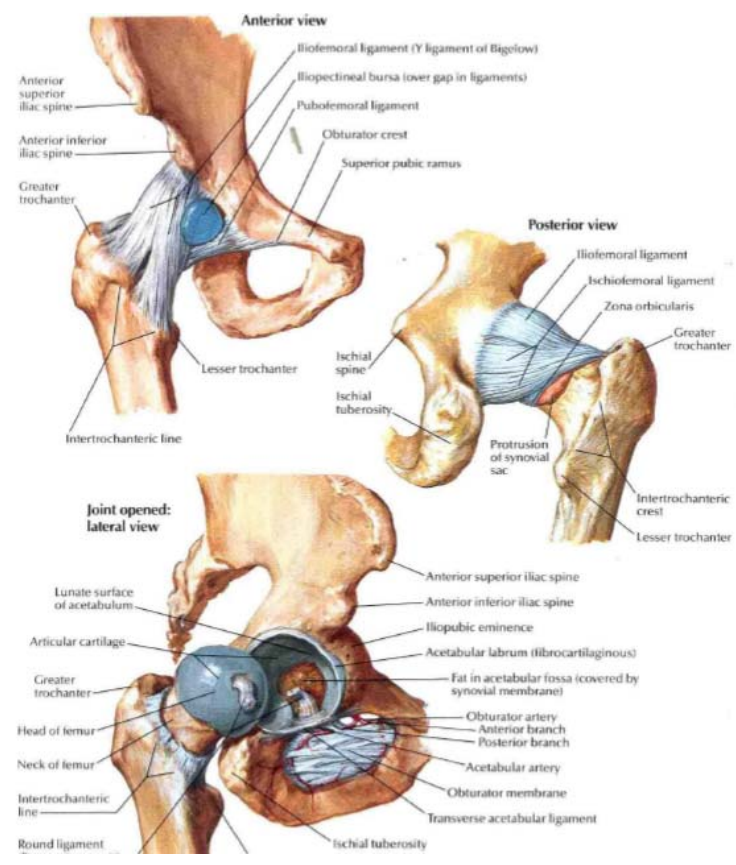

Fig 9 Hip Joint

as level of estrogens decrease, and continues until as much as $30 \%$ of the calcium in bone is lost by age 70 . Once bone loss begins in females, about $8 \%$ of bone mass is lost every 10 years. In males, calcium loss does not begin until after age 60 , and about $3 \%$ of bone mass is lost instead of $8 \%$. The loss of calcium in bones is one of the problems in osteoporosis. The second principal effect of aging on the skeletal system, brittleness, results from a lowered rate of protein synthesis, which diminishes the organic portion of bone matrix, mainly collagen fibers, that gives bone its tensile strength. Inorganic minerals gradually constitute a greater proportion of the bone matrix. The loss of tensile strength causes the bones to become very brittle and susceptible to fractures, as currently many studies proposed about. In some elderly people, collagen fiber synthesis slows, in part due to diminished production of human growth hormone. In addition to increasing the susceptibility to fractures, loss of bone mass also leads to deformity, pain, stiffness, likely loss of height in the human body, and loss of teeth.

\subsection{Hip (Coxal) Joint}

The hip is one of the human joints that allow the greatest mobility. It is characterized by intrinsic stability too, thanks thank to the concave shape of the acetabulum and to the presence of a bony rim in the superior, posterior and anterior aspects [17]. The human hip joint is well constructed for its intended use: standing and walking. The hip joint is an outstanding example of a congruous joint.

\subsubsection{Anatomy of the joint}

A complete overall image of the joint is well depicted in Figure 9 where anterior, posterior and lateral view of
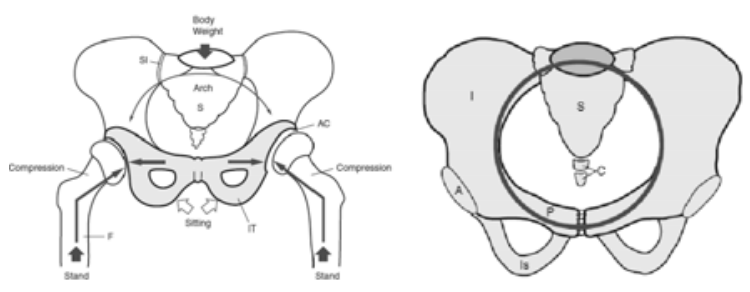

Fig 10 Mechanical view
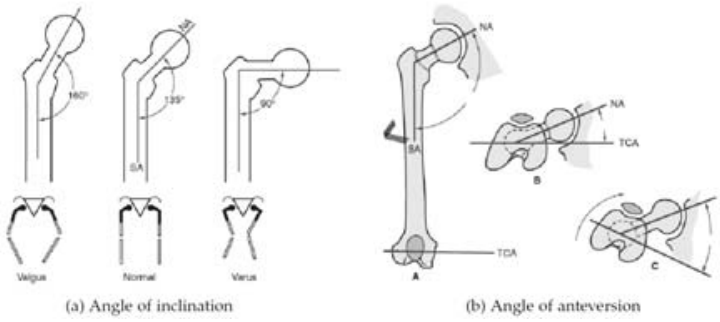

Fig 11 Rotations in hip joint

the articulation have been described in structures components. Both the concave (acetabulum) and the convex (femoral head) are symmetrical, and the joint space is equal at all points with slight deviation to permit adequate lubrication. This symmetry allows for rotation about a fixed axis and simplifies the muscle action on that joint. The weight of the body is superimposed on the fifth lumbar vertebra and then transferred to the base of the sacrum and across the sacroiliac joints to the ilia. When a person is standing, the weight of the body is transferred to the acetabula and finally to the femora. When a person is sitting, the weight is borne on both ischial tuberosities. The femoral head articulates within the acetabulum, which is horseshoe shaped and coated with cartilage around most of its periphery, the center is free of cartilage, the bottom of the "ring" of the peripheral acetabulum is not complete. It is completed as a ring by the transverse acetabular ligament. It is also deepened by a cartilagecovered ring of fibrocartilage termed the labrum. The head of the femur fits into the acetabulum, where it is
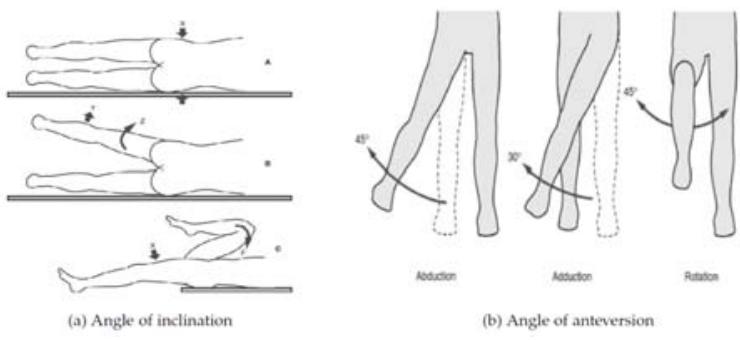

Fig 12 Movements in hip joint 
held firmly by a thick capsule, which is divided into thickened layers forming the iliofemoral, pubofemoral, and ischiofemoral ligaments. In the standing position, the center of gravity passes behind the center of rotation of the hip joint. The pelvis is angled so that the femoral head is seated directly into the acetabulum. The anterior portion of the capsule is thickened to form the iliofemoral ligament. This permits static stance to exist on ligamentous support without supporting muscular contraction. In a toe-out stance, the head of the femur is directed in a forward outward direction. This direction could be one of subluxation except for the support of the iliopsoas muscle tendon rather than from the iliofemoral ligament, which is placed too far laterally for that function. The head of the femur is coated by a cartilage that acts to cushion compressive forces and lubricates the joint during compression. When not bearing weight, the cartilage imbibes nutritional fluid stage.

\subsubsection{Movements}

In the following representation depictions have been reported in order to schematize parts of the joint whose each one has been presented in a meaningful vision of mechanical properties (please refer to Figures 10, 11 and 12). Body weight is borne on sacrum (S) and then transmitted through sacroiliac joints (SI), which form an arch. Weight is then taken to acetabular joints (AC). Ilia form pubic struts, which neutralize force on femora (F). Standing causes compression forces at acetabula, and sitting causes compression forces at ischial tuberosities (IT). Bony structures of pelvis form ring that contains viscera of pelvis. Components of ring include sacrum (S), ilia (I), pubic bones (P), ischia (Is), and acetabula (A). There are several angles of the head and neck of the femur that merit review. The head and neck of the femur, when viewed from the front, are at an angle of inclination. Viewed from above, the femoral head and neck form an angle of anteversion. The extreme stability of the hip joint is related to the very strong articular capsule and its accessory ligaments, the manner in which the femur fits into the acetbulum, and the muscles surrounding the joint. Although the shoulder and hip joint are both ball-andsocket joins, the movement at the hip joint does not have as wide a range of motion. Indeed tithe ranges of motion of the hip joint include flexion, extension, abduction, adduction, and rotation, with specific physiological limitation by the soft tissues of the joint: flexion is limited by the hamstring muscle group extension is limited by the ligamentous thickening of the capsule, abduction by the adductor group of muscles, adduction by the tensor muscle and fascia of the abductor muscles and rotation by the fibrous capsular fibers. Lastly medial rotation is limited by tension in the ischiofemoral ligament, and lateral rotation is limited by the tension in the iliofemoral and pubofemoral ligaments [3].

\subsubsection{Mechanics in hip joints}

Loads acting on the hip vary depending on the variation of body weight, body position and forces externally applied. When the individual is in an upright position body weight is transmitted by L5 lumbar vertebra to the sacral base, sacroiliac joints, and ileum ischium, femurs, tibias until feet. We can start our analysis assuming that: the weight of the upper body acts through the centre of the pelvis, all the body segments are rigid bodies, only the frontal plane is considered (2D) and only the abductor muscles are considered to act. Hip joint biomechanics are quite complex due to pelvic motion associated with it and range of movements it produces. During normal gait, on heel-strike, the hip moves into 3 degrees of flexion and at toe-off (when the foot is finally off the ground) about 10 degrees of extension. The range of abduction to adduction is about 11degrees, and for internalexternal rotation, the range is about 8 . During different phases of gait cycle, different forces act on femoral head. Approximately two thirds of the hip force is produced by the abductors. The directions of the resultant force on the joint are important to the
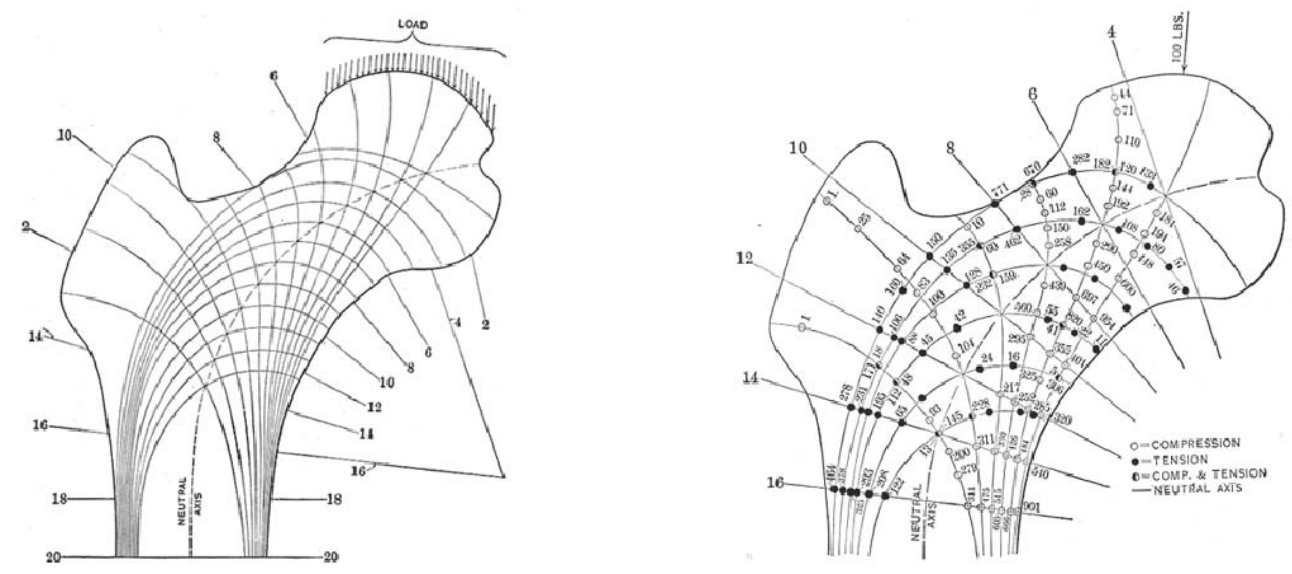

Fig 13 Femur biomechanics 


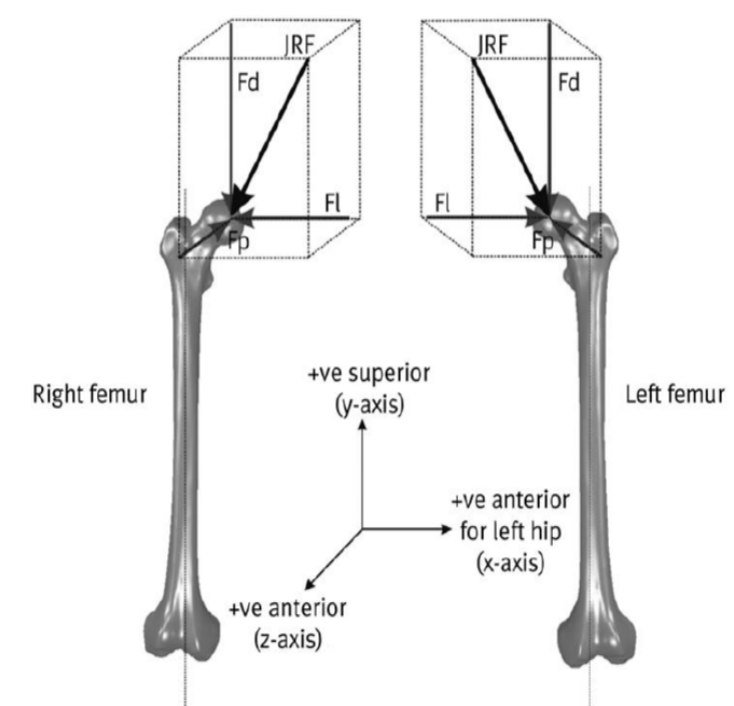

Fig 14 Resultant of forces

function of total hips. It is useful to consider the forces relative to axes based on the long axis of the femur. In the coronal plane the forces acting make an angle of 15 to 27 degrees to the long axis of the femur during stance phase of gait which results in axial compression, varus and mediolateral forces. In the saggital plane, anteroposterior forces on the femoral head, result in torsion. The latter has significant role in the compressive failure of trabecular bone in uncemented stems and resulting in stem fractures. Femoral offset often influences the mechanics of the hip. Femoral offset id often reduced in normal total hip replacement. This results in an increase in the required abductor force leading to a higher resultant joint force and sometimes a gait abnormality. An increase in offset would reduce the force but causes an increase in the bending moment on the stem. Consider a person of mass $\mathrm{m}$. The weight of the person is $\mathrm{mg}$ (mass* acceleration due to gravity). The weight of each leg is $15 \%$ of body weight, the next figure shows a person standing on two legs and bearing equal weight on each leg. The joint reaction force, JRF acts at the hip joint centre; the femur applies a force to the pelvis and the pelvis applies an equal and opposite force on the femur (Newton's third law). The abductor muscle can only act in tension and applies a force A at its attachments to the pelvis and the femur (again equal and opposite due to Newton's third law). There will be two reactions from the ground that act at each of the feet, equal and opposite to the weight of the upper body. Whilst traditional teaching focuses on two-dimensional analyses, as was shown in the example of a person using a walking stick, in reality the musculoskeletal system is three dimensional. An illustration of this is given in Figure 13, which shows the JRF acting on the femur in three-dimensions. There are three mutually perpendicular forces acting on the femur [6]: Fd acts distally, Fp posteriorly and Fl laterally. The total JRF is the resultant of the three forces acting at the hip joint. The direction and magnitude of the JRF varies with activity [6]. The magnitude of the JRF at the hip has been measured to be $300 \%$ of body weight during normal walking, and up to $500 \%$ of body weight whilst jogging. When describing the action of a force on the limb in three-dimensions, it is important to establish a coordinate system and the anatomical directions that relate to it. If a coordinate system is established with the positive $\mathrm{z}$-axis direction anterior, the positive $\mathrm{x}$ axis direction medial and the positive $\mathrm{z}$-axis direction superior, then Fd and Fp will be negative for the left and right femurs. For the right femur, a positive force along the $\mathrm{x}$-axis will act medially whereas for the left femur a positive force along the $\mathrm{x}$-axis will act laterally. Alternatively Fl (defined as acting laterally) will be positive for a left femur and negative for a right femur. In Figure 14 is depicted a diagram of the lines of stress in the upper femur, based upon the mathematical analysis of the right femur. These mechanical behaviors result from the combination of the different kinds of stresses at each point in the bone shape. Subsequently, in 14b it has been shown the intensity of the maximum tensile and compressive stresses in the upper femur. Computed for the load of 100 pounds on the right femur from the study [45].

\section{THR AND IMPLANTS}

Hip replacement surgery is the second most common joint replacement procedure, closely following knee replacements, and year by year in continuous increasing all over Europe countries, as shown in Figure 15. The surgery is performed when the hip joint has reached a point when, due to different diseases and injuries, painful symptoms can no longer be controlled with non-operative treatments. In a hip replacement procedure, surgeons remove the damaged joint surface and replace it with an artificial implant.

An important analysis instrument has been constituted in UK: the National Joint Registry (NJR) was set up by the Department of Health and Welsh Assembly Government to collect information on all hip, knee and ankle replacement operations and to monitor the performance of replacement hip, knee and ankle joints (implants). Beside British data, NJR provides us statistics from the European community in collaboration with Eurostat [35]. Osteoarthritis in advanced stadium of gravity leads mainly to the THR, this is the most prevalent chronic condition and is a leading cause of disability. However, the impact of arthritis is not restricted by national boundaries, affecting an estimated 3 million Australians, 6 million Canadians, 8 million British, almost 43 million Americans and 103 millions in Europe. Nevertheless there are too few comparable studies on the prevalence of osteoarthritis in Europe to draw any conclusions on cross-country variations (Figure 15). Nor is there any evidence as to whether the age- and sex specific 

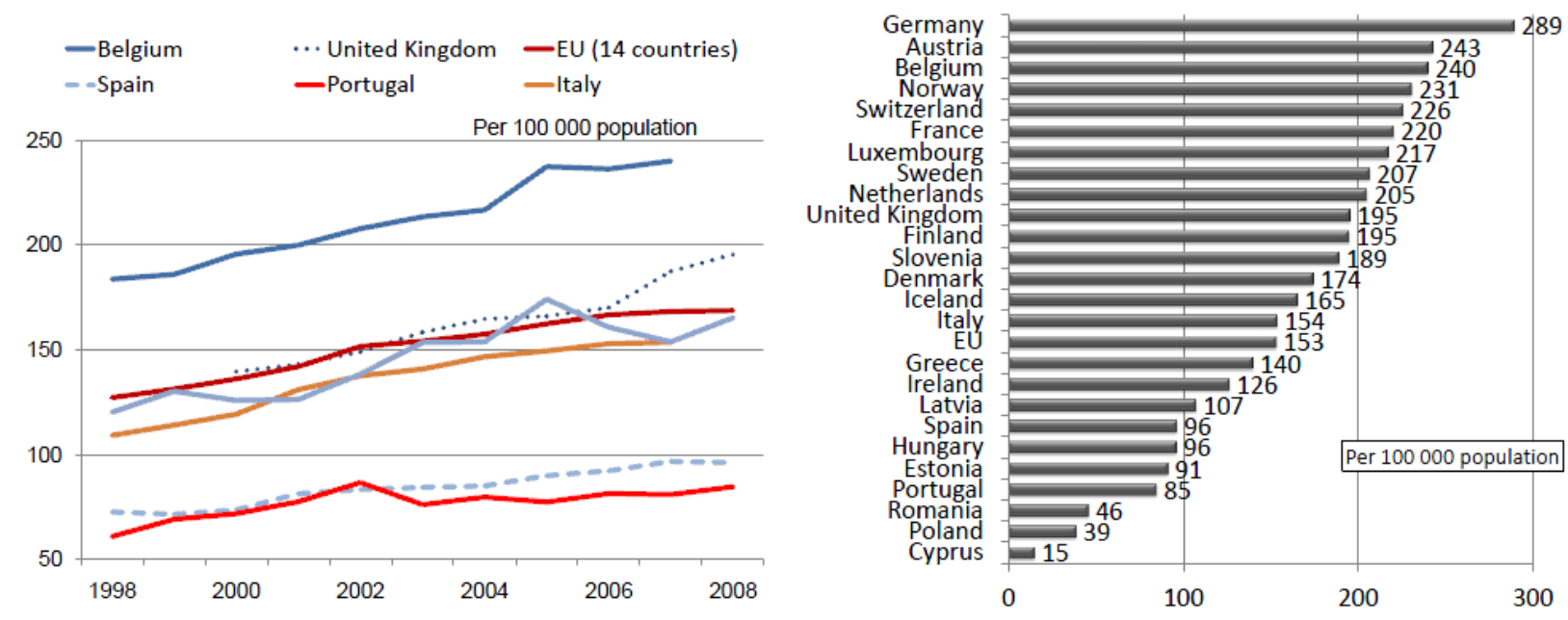

Fig 15 Trends of hip replacement surgery

incidence of osteoarthritis has changed in recent decades, indeed the number of people suffering from osteoarthritis has increased, and is expected to continue to increase in the coming years, for two reasons: population aging, which is resulting in a growing number of people over 60 and 65 years with a greater risk of suffering from osteoarthritis (even if the age and sex specific rate does not increase); and the growing prevalence of obesity, which is the main risk factor for osteoarthritis beyond age and sex (European Commission). Definitely these numbers presented in Table 2 due to a broad range of disabilities are intended to increase. The THR procedure is often executed in hospitals of all Europe and represents an important economic effort for European health systems. Because of the population aging and improvements of the surgery technique, work volumes are increasing year by year. Regarding the Italian context, the analysis of SDO database (Scheda di dimissione ospedaliera) shows an increment of $32.4 \%$ in THR treatments (COD: ICD-9-81.51), as well as depicted in Figure 16, performed under any diagnosis in 1999-2005.

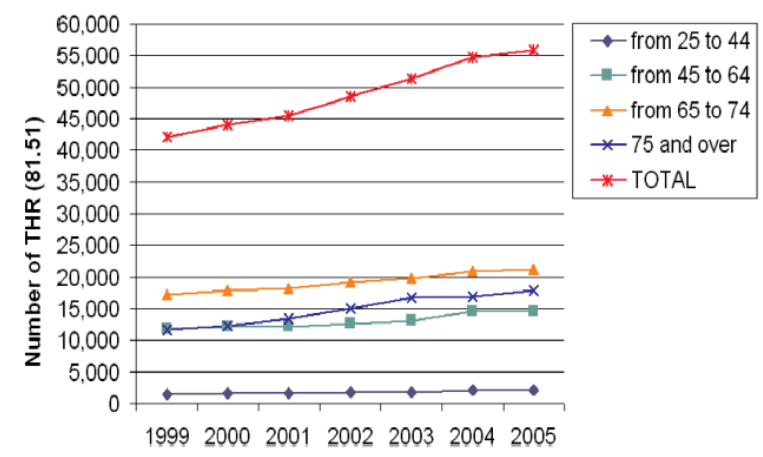

Fig 16 Data from SDO databases
The class of people much involved are not "75 and over" as expected from many studies. The analysis indeed identifies 65 to 74 years individuals as main interested in THR, in prevalence females rather than males [14]. However, the accumulative amount of treatments is in growth as factors like aging impend.

\subsection{Operation intro}

Over the last decades, total hip arthroplasty (THA) has become one of the most successful surgical operations. Symptoms such as pain and loss of function indicate THA as the solution. These symptoms can appear in different forms, as rigidity, deformity, limb shortening or movement reduction. THA is the solution preferred also in case of osteoarthritis, rheumatoid arthritis, psoriac arthritis, avascular necrosis, trauma and tumors. According to MedlinePlus, a service of the U.S. National Library of Medicine and the National Institutes of Health, although the hips are stable, there are numerous diseases or conditions that affect the hip's ball-and-socket joint, including Legg-CalvePerthes disease, congenital hip dislocation and osteoarthritis [1]. Hip joint diseases can cause pain, decrease range of motion and reduce a person's quality of life. Briefly are listed the main diseases according with subsequently reported statistics. Legg Calve Perthes Disease: The Mayo Clinic states that Legg Calve Perthes disease occurs during childhood, is a degenerative condition and is associated with insufficient blood supply to part of the hip joint. LCP disease is characterized by avascular osteonecrosis (death of bone tissue due to an interruption in normal blood supply) of the capital femoral epiphysis of the femoral head. the bones become unstable, causing them to break easily and heal slowly. The Mayo Clinic states that LCP disease often affects just one hip, although it occasionally manifests in both hips, and that it's most common among those between the ages of 2 and 12. Children who develop the disease at a 
Total hip replacement: structures modeling, gait analysis and report

European Journal Translational Myology - Basic Applied Myology 2012; 22 (1\&2): 69-121

\begin{tabular}{ccccccccccc} 
& 1999 & 2000 & 2001 & 2002 & 2003 & 2004 & 2005 & 2006 & 2007 & 2008 \\
\hline Austria & & & & & & & & 234,4 & 236 & 242,8 \\
$\begin{array}{c}\text { Belgium } \\
\text { Cyprus }\end{array}$ & 185,9 & 195,6 & 199,9 & 207,7 & 213,4 & 216,6 & 237,3 & 236,2 & 240 & \\
Denmark & 149,4 & 159,2 & 163,4 & 188,1 & 182 & 191,6 & 194 & 200,5 & 199,6 & 174,3 \\
Estonia & & & & & & & & & & 91,2 \\
Finland & 143,7 & 149,3 & 152,8 & 165,4 & 171,5 & 161,8 & 184,5 & 191,2 & 181,2 & 194,9 \\
France & 205,3 & 207,3 & 212,2 & 215,5 & 214,9 & 212,2 & 217,1 & 215,6 & 217,7 & 220,4 \\
Germany & & & & & & & 263 & 270,3 & 280,2 & 289,3 \\
Greece & 61,2 & 73,2 & 88,5 & 101,4 & 119 & 126,4 & 119,5 & 139,8 & & \\
Hungary & & & & & & 99,2 & 102,8 & 100,5 & 91,3 & 96 \\
Iceland & 130,2 & 125,9 & 126,3 & 138,4 & 153,5 & 153,8 & 174,1 & 160,7 & 153,8 & 165,3 \\
Ireland & 123,1 & 133,6 & 127 & 133,4 & 133,7 & 134,8 & 140,5 & 138,7 & 130,7 & 126,4 \\
Italy & 114,1 & 119,3 & 131 & 137,6 & 140,8 & 146,7 & 149,4 & 152,9 & 153,6 & \\
Latvia & 67,0 & 63,6 & 68,0 & 68,7 & 71,2 & 75,2 & 83,2 & 104,9 & 98,7 & 107,2 \\
Luxembourg & 190,7 & 185,7 & 192,7 & 212,3 & 210,3 & 215,5 & 208,3 & 200,6 & 217,3 & \\
Netherlands & 165,9 & 167,1 & 173,7 & 183,4 & 189,5 & 192,9 & 198,3 & 201,4 & 205,1 & \\
Norway & & 171,4 & 186 & 187,1 & 211,5 & 192,1 & 202,8 & 197,1 & 230,7 & \\
Poland & & & & & 27,1 & 24,5 & 26,2 & 32,5 & 33 & 38,6 \\
Portugal & 69,2 & 71,9 & 77,6 & 86,7 & 76,2 & 79,8 & 77,4 & 81,5 & 81 & 84,7 \\
Romania & & & & & & & & & & 45,7 \\
Slovenia & & & & & & 147,2 & 160,5 & 178,6 & 168,6 & 189,1 \\
Spain & 71,6 & 73,7 & 81,4 & 83,4 & 84,4 & 85,1 & 90 & 92,3 & 96,8 & 96,2 \\
Sweden & 151,8 & 166,3 & 176,4 & 189,9 & 191,7 & 200,9 & 208 & 207,4 & 206,7 & \\
Switzerland & & & & 192,7 & 200,8 & 210 & 218,4 & 226 & 232 & 225,6 \\
United Kingdom & & 139,6 & 143,1 & 149 & 158,3 & 164,7 & 166 & 170,1 & 187,4 & 195,4 \\
EU (14 countries) & 131,3 & 136,1 & 142,0 & 151,6 & 154,1 & 157,4 & 162,4 & 166,6 & 168,3 & 168,7 \\
\hline \hline
\end{tabular}

Table 2 Trend in hip replacement surgery, 1998 to 2008 (or nearest year available), selected countries

young age have the best prognosis and the more time there is to re-form the involved hip bone. Anyway the majority of children with LCP disease go on to lead normal lives. Congenital Hip Dislocation: CHD, has an incidence of 1.5 per 1,000 births, and is eight times more common in girls than boys.

The left hip is affected twice as often as the right, and that bilateral dislocation occurs in greater than $25 \%$ of cases. To rule in a diagnosis of CHD, certain physical exam and radiographic findings must be present. Clinical indicators that suggest CHD in newborns or infants include a limited ability to move a flexed hip away from the body, one leg shorter than the other and several positive orthopaedic tests. Radiographic measurements to screen for CHD include the following: Hilgenreiner line, acetabular index, PerkinOmbredanne line, Shenton- Menard line and Andrenvon Rosen line.

CHD often can be treated using conservative care, especially if it's caught early. Osteoarthritis: The American Academy of Orthopaedic Surgeons, or AAOS, states that, like the other weight-bearing joints in the body, the hips may be vulnerable to osteoarthritis, which is the most common form of arthritis. Osteoarthritis, also known as degenerative joint disease or DJD, involves the degradation of joints, including the excessive wearing of the smooth articular cartilage on the end of bones [34]. Some of the first symptoms associated with hip joint osteoarthritis are discomfort and stiffness in the groin, buttocks or thighs upon waking in the morning. Osteoarthritis is characterized by acute flare-ups during bouts of activity that are relieved with rest and without appropriate intervention progresses to the point that rest is no longer effective in reducing osteoarthritisrelated pain. The hip joint then becomes stiff and inflamed, and bone spurs, also known as osteophytes, may accumulate along the joint's edges. In advanced cases, there is bone-on-bone contact, which can produce severe pain and discomfort and limit activity. Decreased activity leads to weak muscles and an overall reduced quality of life [19]. Fractures of the acetabulum occur primarily in young adults as a result of high velocity trauma. These fractures are often associated with other life-threatening injuries. Displacement of the fracture fragments leads to articular incongruity of the hip joint that results in abnormal pressure distribution on the articular cartilage surface. This can lead to rapid breakdown of the cartilage surface, resulting in disabling arthritis of the 


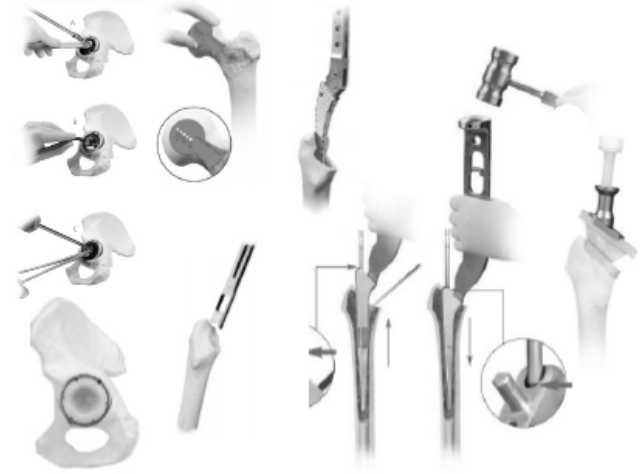

(a) From the acetabular insertion to prosthesis fitting
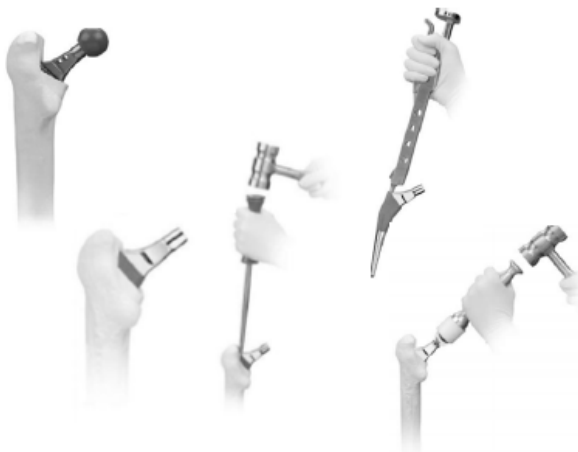

(b) Rasping, fizing and acetabular connection

Fig 17 Surgeons workflow

hip joint. Anatomic reduction and stable fixation of the fracture, such that the femoral head is concentrically reduced under an adequate portion of the weight bearing dome of the acetabulum, is the treatment goal in these difficult fractures and studies authors concluded that in appropriately selected patients THA can be an acceptable treatment approach [10]. The first step of a hip replacement surgery is to remove the injured cartilage and bone. When the hip joint becomes arthritic, the normally smooth cartilage surface is worn away. To re move the worn out ball of the ball-andsocket hip joint, the bone is cut to remove the femoral head and in order to insert a new joint, the damaged bone and cartilage must first be removed. Once the arthritic ball is removed, the worn out socket can be addressed. Unlike the ball, this bone cannot be cut off because the socket of the hip joint is part of the pelvis bone. Thus a special tool called a reamer, aiming the discharge of the hip socket arthritis, is used to scrape away the cartilage and bone [15]. This leaves a smooth, perfectly rounded surface to accept the new hip replacement implant. Once the damaged bone has been removed from the acetabulum, the new socket of the hip replacement can be inserted. The acetabular component is held tightly in the pelvis by making the socket slightly smaller than the acetabular component, and wedging the implant into the bone. The implant
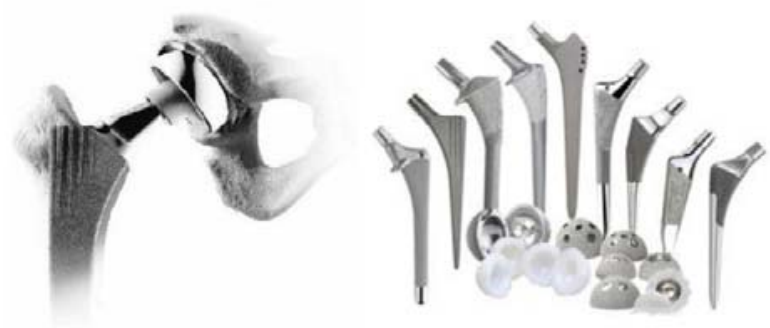

Fig 18 Implants has a rough surface to allow bone to grow into the surface of the implant over time. The ball is supported with an implant inserted down the hollow center of the thigh bone (femural stem); like the acetabular socket, the femoral stem must be held tightly in the bone and gain special tools are used to shape the center of the thigh bone to accommodate the femoral stem. With the bone prepared to accept the stem of the hip replacement implant, the femoral stem is inserted. The stem can be held in the bone with or without cement. When the bone is held with cement, the cement is inserted in a liquid form, and the stem is then placed. Within a few minutes, the cement permanently hardens to hold the implant fixed within the bone. When no cement is used, the implant is called "press-fit", meaning that the implant is wedged tightly into the bone and a rough surface covering the implant allows bone to grow into the implant over time. With the stem inserted down the center of the thigh bone, the ball of the ball-and-socket hip joint can be inserted on top of the stem, the metal ball is tightly fitted onto the top of the stem. Hence the hip replacement can be placed in final position with the parts in place including the ball into the socket.

\subsection{Hip joint prosthesis}

The aim of THA is to reproduce in the hip and in the femur the same load transfer and the same stress
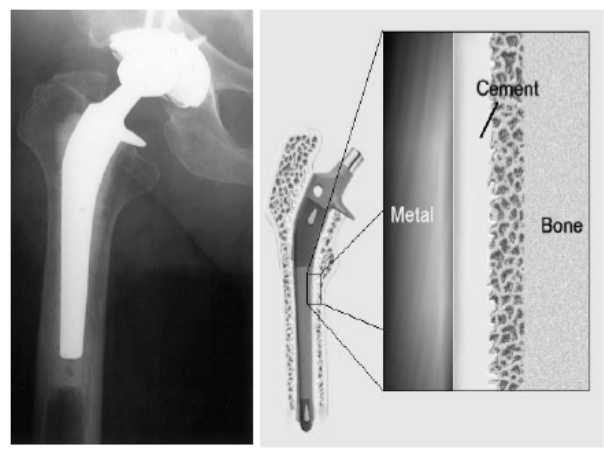

Fig 19 Cemented implant 
distributions of the healthy femur. The forces normally applied to the femur across the hip joint are transmitted via the cancellous bone of the pelvis to the acetabular subchondral bone. These forces can have resultants being in magnitude several times the body weight. The forces are shared by the complex network of cancellous bone of the femoral head and of the femoral neck, and are then conveyed to the femoral shaft. We have already highlighted how the wall head and shaft architecture is functional to the load that hip joint and the femur are designed to bear. Results obvious that the drastic modification in shape, mass and mechanical characteristics of the structures after total hip arthroplasty deeply alter the equilibrium between form and functions of the hip [9]. After uncemented total hip arthroplasty, a material having uniform stiffness and strength replaces the complex subchondral plate of the acetabulum [18]. In the femur, the proximal shaft is filled by a large, stiff piece of metal, having dimensions and mechanical characteristics that respond to the needs of having a highly strong filling (to prevent femur fracture) and a strong bone implant interface. The presence of a stiff, heavy filling results in a drastic increase of the overall stiffness of the proximal femur. If the material is too stiff and the proximal fit produced during surgery by pressfit is not quasi-perfect, then the risk of stress shielding increases. Stress shielding is due to the fact that the higher stiffness of the metal leads the stem to uptake the most of the load transferred to the femur thought the hip joint [29]. Because the proximal femur is no more loaded as before, it remodels, loosing a significant mass of cortical bone. At the same time, the stem transfers the load uptake proximally to the bone of the femoral shaft, in correspondence of its distal tip (Figure 17). The consequence is an evident highpertrophy of the femoral shaft, at the level just distal to the tip of the stem. If the proximal resorption is massive, the process yields mobilization, strength reduction and on the long term gross loosening. Many people credit Sir John Charnley, a British orthopaedist, with performing the first modern total hip replacement. His innovations included combining a metal stem and ball with a plastic shell and using a methacrylate cement to hold the devices in place. Today, the stem portions of most hip implants are made of titanium or cobalt/chromium-based alloys (Figure 18). They come in different shapes and some have porous surfaces to allow for bone ingrowths. Cobalt/chromium-based alloys or ceramic materials (aluminum oxide or zirconium oxide) are used in making the ball portions, which are polished smooth to allow easy rotation within the prosthetic socket. The acetabular socket is made of metal, ultra-high molecular-weight polyethylene, or a combination of polyethylene backed by metal. All together, these components weigh between 14 and 18 ounces, depending on the size needed. All the materials used in a total hip replacement have four characteristics in common. Biocompatibility: They can function in the body without creating either a local or a systemic rejection response. Resistance to corrosion, degradation, and wear: Therefore, they will retain their strength and shape for a long time. Resistance to wear is particularly significant in maintaining proper joint function and preventing the further destruction of bone caused by particulate debris generated as the implant parts move against each other. Mechanical properties: In order to duplicate structures it is supposed they have to replace, they are strong enough to withstand weight-bearing loads, flexible enough to bear stress without breaking, and able to move smoothly against each other as required. Highest standards: Implants standards established by healthy community extend to fabrication and quality control at a reasonable cost. The project THR aims to get an instrument of support in order to adapt the choice about the type of implant to different patients. Besides prototypes in development which are focused on hybrid systems, the available options are mainly cemented and uncemented implants, thus this kinds of prosthesys will be detailed in the next lines.

Cemented total hip replacement: Over the past 40 years, there have been many improvements in both the materials and the methods used to hold the femoral and acetabular components in place. Today, the most commonly used bone cement is an acrylic polymer called polymethylmethacrylate (PMMA). A patient with a cemented total hip replacement can put full weight on the limb and walk without support almost immediately after surgery, resulting in a faster rehabilitation.

Briefly in the cemented approach to hip joint replacement, bone cement is used within the femoral canal to help secure the implant in position (see Figure 19, 20). The PMMA or others are types of grouting material between the bone and implant. Centering the implant within the femoral canal and the cement mantle is very important for stability because it helps

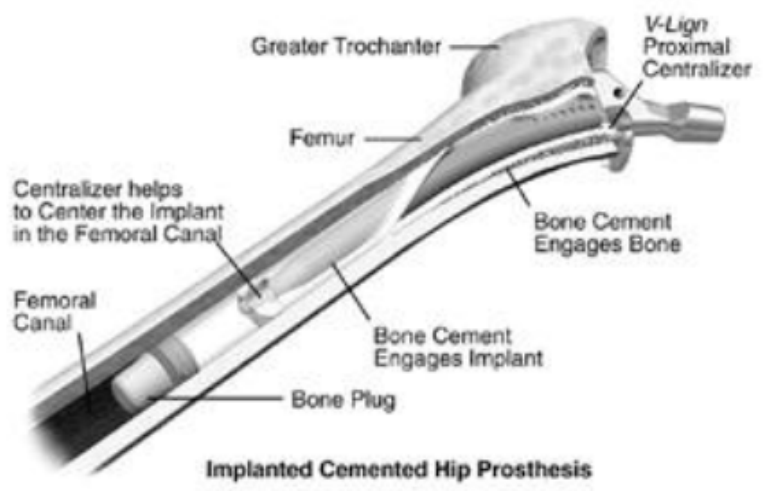

Fig 20 Inner structure 
distribute stresses evenly. For example Zimmer offers centralization devices to help the physician achieve centralization at the top and bottom of the replacement hip joint. The VerSys System's cemented hip option provides the physician the opportunity to create patient specific solutions. Although cemented implants have a long and distinguished track record of success, they are not ideal for everyone.

Cemented fixation relies on a stable interface between the prosthesis and the cement and a solid mechanical bond between the cement and the bone. Today's metal alloy stems rarely break, but they can occasionally loosen. Two processes, one mechanical and one biological, can contribute to loosening. In the femoral component, cracks (fatigue fractures) in the cement that occur over time can cause the prosthetic stem to loosen and become unstable. This occurs more often with patients who are very active or very heavy. The action of the metal ball against the polyethylene cup of the acetabular component creates polyethylene wear debris. The cement or polyethylene debris particles generated can then trigger a biologic response that further contributes to loosening of the implant and sometime to loss of bone around the implant. The microscopic debris particles are absorbed by cells around the joint and initiate an inflammatory response from the body, which tries to remove them. This inflammatory response can also cause cells to remove bits of bone around the implant, a condition called osteolysis. As the bone weakens, the instability increases. Bone loss can occur around both the acetabulum and the femur, progressing from the edges of the implant. Despite these recognized failure mechanisms, the bond between cement and bone is generally very durable and reliable. Cemented total hip replacement is more commonly recommended for older patients, for patients with conditions such as rheumatoid arthritis, and for younger patients with compromised health or poor bone quality and density. These patients are less likely to put stresses on the cement that could lead to fatigue fractures.
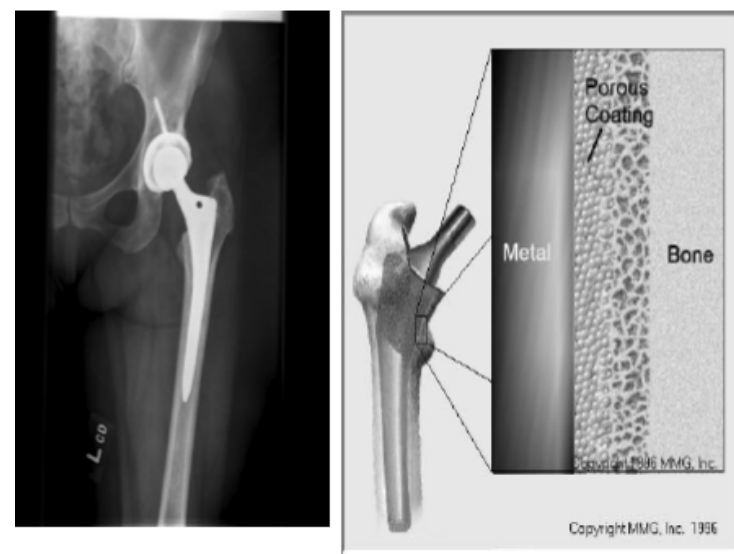

Fig 21 Uncemented implant
Cementless Total Hip Replacement: In the 1980s, new implant designs were introduced to attach directly to bone without the use of cement. In general, these designs are larger and longer than those used with cement. They also have a surface topography that is conducive to attracting new bone growth. Most are textured or have a surface coating around much of the implant so that the new bone actually grows into the surface of the implant. Because they depend on new bone growth for stability, cementless implants require a longer healing time than cemented replacements. The orthopaedic surgeon must be very precise in preparing the femur for a cementless impact. The implant channel must match the shape of the implant itself very closely. New bone growth cannot bridge gaps larger than $1 \mathrm{~mm}$ to $2 \mathrm{~mm}$.

Cementless femoral components tend to be much larger at the top, with more of a wedge shape. This design enables the strong surface (cortex) of the bone and the dense, hard spongy (cancellous) bone just below it to provide support (refer to Figure 21). The acetabular component of a cementless total hip replacement also has a coated or textured surface to encourage bone growth into the surface. Depending on the design, these components may also use screws through the cup or spikes, pegs, or fins around the rim to help hold the implant in place until the new bone forms. The pelvis is prepared for a cementless acetabular component using a process similar to that used in a cemented total hip replacement procedure. The intimate contact between the component and bone is crucial to permit bone ingrowth. Although some orthopedic surgeons are now using cementless devices for all patients, cementless total hip replacement is most often recommended for younger, more active patients and patients with good bone quality where bone ingrowth into the components can be predictably achieved. Individuals with juvenile inflammatory arthritis may also be candidates, even though the disease may restrict their activities. The bone-metal interface is the most delicate site of an uncemented implant, where the living tissue and the non living material meet each other. Even if the bone is a living material, while the prosthesis is a non living one, these two systems exchange chemical and mechanical signals across the interface. Such signals generate then reactions on both the bone and the implant. After stem implantation, the wounded bone comes in contact with the implant. If the surgery was well performed, the stem is pressed towards the cortical bone on a uniform way along its length and around its sections. The direct contact between the stem and the cortical bone is guaranteed during surgery, when spongy bone is rasped away. Marrow is a soft tissue that cannot support load; neither transmits it to the stem. Moreover it prevents spongy bone to directly growth into the stem. Thanks to this press-fit achieved during surgery, the stem stability is guarantee in the immediate post- 


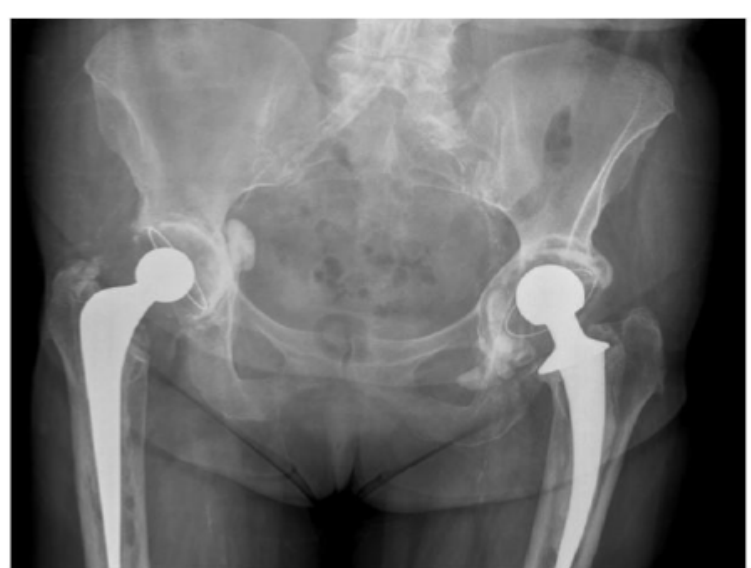

Fig 22 Bilateral arthroplasty

operative period (primary stability), while the bone layers immediately adjacent to the stem are preloaded, thus encouraged to grow. During the first postoperative days the patient is prevented from moving, then gradually higher loads are applied to the operated limb. Time has actually to be given to the wounded bone to overcome the traumatic surgical event: the damaged trabeculae and Haversian systems are removed by the osteoclasts and macrophages and new woven bone is formed by the osteoblasts. The woven bone gradually replaces the bone clot formed around the stem, growing on it and tightly ensuring it in its position (secondary stability). Progressive loading is a key-factor for secondary healing. If not loaded the bone is not stimulated to grow and resorbs and reduces its mineral content. Load allows bone to model and remodel around the stem in a way that best accommodates the new conditions, which were generated after THA. After the operation proportions between the filling material and the outside wall one are inverted with respect to the pre-operative situation (Figure 22).

Bone-Implant interface, unsolved problem: Aseptic loosening and periprosthetic osteolysis are major problems for which a solution has not been found yet. Any type indeed of joint gliding surface continuously produces wear debris, which are derived from implant materials, fragmented bone cement, ceramics and metals. Currently generation of debris is still inevitable, although modern technology provides better biocompatible implant to lessen the debris. Initially, it was hoped that cementless total hip replacement would eliminate the problem of bone resorption or stem loosening caused by cement failure. Even if certain cementless stem designs have excellent long-term outcomes, cementless stems can loosen if a strong bond between bone and stem is not achieved. Theoretically, if the mechanical stress is properly transferred to the bone present around the implants, they can support the joint function without loosening

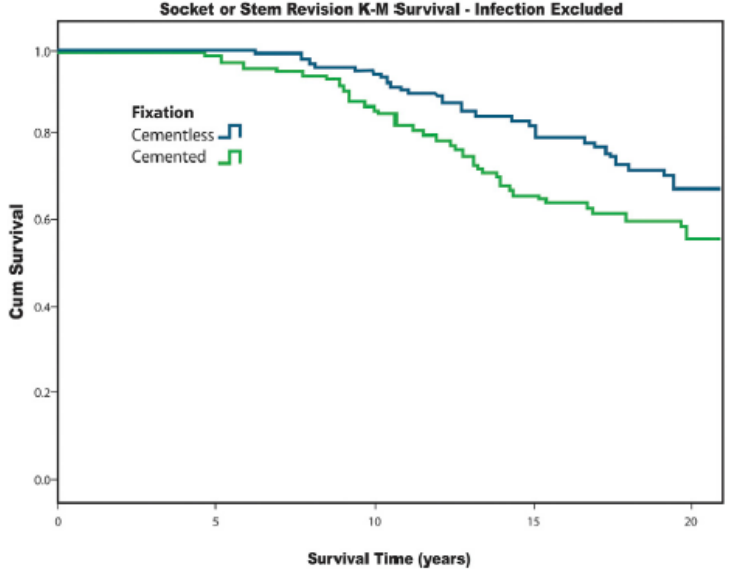

Fig 23 Results of the trial

and causing periprosthetic osteolysis, which guarantees a longer and better survivorship but unfortunately the degradation occurs even if the insertion of the prosthesis is well performed. A ten-year survival rate of approximately $90 \%$ (Figure 23) has been demonstrated for total hip joint surgery and some of them have to be revised due to the problems treated, otherwise a foreign body host response, always including adaptive and reactive processes are inducted [30].

For the future? There is an increasing concern on wearresistant materials, implants to obtain proper osseointegration, and to maintain adequate joint function. Although etiology of periprosthetic osteolysis is still complicated, intensive study on bone-implant interface biology provides various information on periprosthetic bone remodeling to improve bone integration of implants as well as a better understanding of osteolysis in failed artificial total hip joints [44]. Improvements in the wear characteristics of newer polyethylene, and research into newer bearing surfaces as well may help resolve some of these problems in the future in both cemented and uncemented designs. The choice The main debate remains concerning the best fixation method for the components of the implant and it is also the target of the project. The purpose in most of the studies in the literature concerning this matter is to compare the long-term outcomes following total hip arthroplasty with and without cement, considering patients treated with THR after certain years of following up.

It is likely due to difficulties in reaching significant samples or keeping same conditions of measurement long time far from each other or other reasons, but it is anyway evident that the answer to this question is not achieved yet. Concerning the bone-implant interface we already saw that both of the options lead irremediably to biological problems. Because each person's condition is unique, the doctor and patient must weigh the advantages and disadvantages to 
decide which type of prosthesis is better: for some people indeed an uncemented prosthesis may last longer than cemented replacements because there is no cement that can break away. And, if the patient needs an additional hip Replacement (which is likely in younger people), also known as a Revision, the surgery sometimes is easier if the person has an uncemented prosthesis. The primary disadvantage of an uncemented prosthesis is the extended recovery period. Because it takes a long time for the natural bone to grow and attach to the prosthesis, people with uncemented replacements must limit activities for up to 3 months to protect the hip joint. The process of natural bone growth also can cause thigh pain for several months after the surgery. Research has proven the effectiveness of cemented prostheses to reduce pain and increase joint mobility as results usually are noticeable immediately after surgery. Cemented replacements are more frequently used than cementless ones for older, less active people and people with weak bones, such as those who have osteoporosis. An interesting and recent trial, whose statistic outcome has been depicted in Figure 23, can be mentioned for proper meaning. It regards the Journal of bone and joint surgery, named "Comparison of THR with and without cement", handles a sample of fifty-two patients in a group underwent cementless prosthesis surgery and forty-one patients treated by cemented implants. In detail they were analyzed at a minimum of seventeen years of follow-up (mean, 19.5 years; range, seventeen to twenty-one years). The follow-up results in major reliability for cementless implants, "cum survival" and "survival time" expressed in years. A list of clinical advantages and disadvantages can be realized:

Cement THR advantages: The cemented total hip replacement tolerates small deviations from the precise operation technique. The bed cut for the prosthesis in the skeleton need not to be very exact because the bone cement filler will level out all incongruities. The patients can put weight on their new total hips immediately after the operation (in theory). Actually, the strength of the fixation of the cemented total hip to the skeleton is most strong at the end of the operation. The factor that limits full weight bearing is the surgical damage to the soft tissues around the total hip. These tissues must heal before the full weight bearing is possible. The cement layer also acts as an intermediate bumper between the very stiff metal of the total hip prosthesis and the weak skeleton. This bumper levels the peak forces acting on the skeleton around the total hip during gait. Cement THR disadvantages: Pressing the doughy bone cement into the raw bone marrow cavity during the operation may cause circulatory disturbances. The bone cement ages, cracks, and after some time the bond between the prosthesis and the skeleton may be lost.

Uncemented THR advantages: The surgeon avoids all problems with cementing the total hip during the operation (problems with mixing the bone cement, waiting for hardening of the doughy bone cement, changes of the position of prosthetic components while the bone cement is still in doughy state, risk of blood pressure fall and heart failure during cementing of the prosthesis). The patient avoids the risk that the bone cement layer will crack and successively disintegrate years after the operation. Uncemented THR disadvantages: There is a risk that chunks of the bone marrow substance will be pushed into the circulation during the forceful hammering of the cementless total hip into place. The need for restricted weight bearing 6-12 weeks (not always). Pain in the thigh, sometimes major then 1 year. Risk of a fracture of the skeleton during operation, when the surgeon blows the total hip too vigorously into an undersized bony bed. Loosening of the metallic balls or fibers from the porous coated surface. These balls may land inside the total joint between the bearing joint surfaces and act as a third body. These hard metallic balls/fibers then accelerate the wear from the surfaces Each kind of implant, deriving from various constructors, has peculiarities and golden points. Please refer to the appendix A to get vision of adopted implants in the hospital, provided by Zimmer. Given that it is severe to making observations for all the implants on the market, anyway reasonable connections between patient's anamnesis and the final choice for the implant might be obtained as already said.

\section{MODELS}

\subsection{Introduction}

The modeling work is completely based on CT-Scans got before and after operations. The scans are made according to a conventional examination of bone pathology in the AXI plane from the anterior superior iliac spine to the middle of the femur. They are taken with a distance of $0.625 \mathrm{~mm}$ between slices, resulting in a total of about 750 to 800 CT slices, depending on the patient's size. Each slice has $512 \times 512$ pixels, and each pixel has a grey value in the Hounsfield scale of 4096 grey-scale values, meaning that it is represented with a 12-bit value. A total data set from a single scan is therefore $(512 \times 512 \times 750 \times 12 \times 700) / 8=2,75 \times 108$ bytes (considering that 2 bytes are needed for 12 bit representation then a data set of this type is roughly 550 MB. This data set gives a complete threedimensional description of the upper femoral and acetabulum bone. Hence, images in the standard format of dicom are imported in Mimics Materialize, a modeling software later discussed, to create virtual structures representing object of study. Since the software handles all the slices, the segmentation can begin. This phase allow to get information about the prosthesis implanted and make 3D models useful for next steps of analysis. The modeling and the final modeling analysis can be held, to get models of human bones and muscles considered, and subsequently 
Ansys is used by engineers in particular to mimic applied loads on certain parts basically through the finite element model analysis, yielding forces response. The chapter is organized in the following manner: methods are reported first of all, detailing the instruments utilized and the considered protocols; the elaboration and partial significant results are in particular analyzed in this section and furthermore the theory on which all the modeling studies are based. Mimics Materialize: Mimics is a software specially developed by Materialise for medical image processing. Mimics is properly utilized for the segmentation of 3D medical images (coming from CT, MRI, microCT, CBCT, Ultrasound, Confocal Microscopy) and the result will be highly accurate 3D models of anatomy of patients.

It is supposed that the obtained results, these patientspecific models, are useful for a variety of engineering applications directly in Mimics or 3-matic, or export the 3D models and anatomical landmark points to 3rd party software, like statistical, CAD, or FEA packages. The "Mimics Innovation Suite" has visualization options for virtual 3D anatomy, but sometimes there is nothing more powerful than getting hands on a physical model. For this, There is an Additive Manufacturing to create accurate, realistic, and tangible models. Physical models allow exploring and evaluating patient anatomy to gain better insight into specific pathology. This knowledge facilitates medical diagnoses. 3D models are also an excellent tool for discussing treatments with patients or the medical team as well as in our hospital biomedical engineers are doing in particular on maxilla surgery. They even allow bending plates and fitting implants to the model prior to surgery. Materialise can manufacture parts elaborated in a wide variety of materials according to the customer needs. Ansys: The ANSYS Workbench platform is the framework upon which the industry's broadest and deepest suite of advanced engineering simulation technology is built. An innovative project schematic view ties together the entire simulation process, guiding the user through even complex multiphysics analyses with drag-and-drop simplicity. With bi-directional CAD connectivity, powerful highly-automated meshing, a project level update mechanism, pervasive parameter management and integrated optimization tools, the ANSYS Workbench platform delivers unprecedented productively, enabling Simulation Driven Product Development. ANSYS is definitely one of the world's leading developers of engineering simulation software, used by product designers and engineers around the world. The Company's open and flexible solutions enable analysis of designs against real-world conditions on a desktop computer. This technology supports quick, efficient and cost-effective product development throughout the development process (from design concept to finalstage testing, validation and production). The ultimate result is successful, innovative products and a faster return on investment for those companies who choose software from ANSYS.

\subsection{Modeling}

Preop and Postop images from the hospital are now available and free to be managed. Using post-op images 3D object has been created based on the implant used in the operation, divided in head and stem, artifacts free, in order to mimic the virtual operation on the femur. Despite different human tissues and implants material can be distinguished in CT slices, gray value thresholds have to be considered. Several times the use of bands of HU which surround intervals of suited values has been adopted because of better result, yielding different colored mask for implant and tissues. A 3D object has been realized and then smoothed with standard parameters established in order to fit this new implant in the femur model coming from preop images.
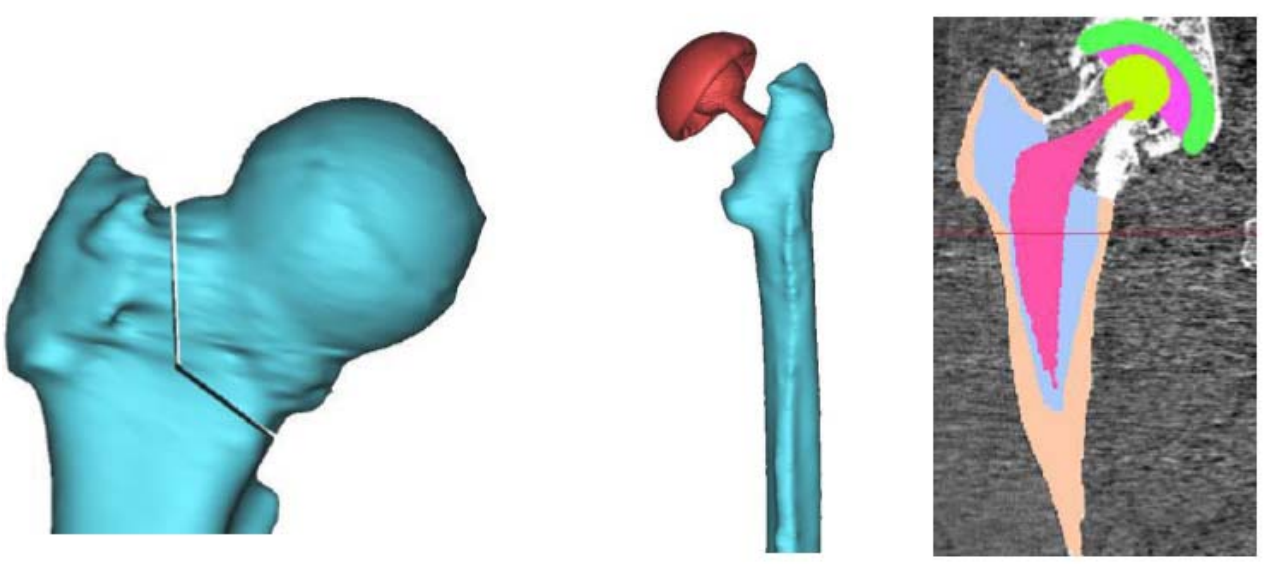

Fig 24 Final meshing result 
4.2.1 Image segmentation: In digital imaging, the image segmentation is the process of partitioning the screen view into meaningful regions. This selection has been properly done even though a loss of information has to be expected, finalized to one more compact representation easier to be examined and managed by image processing tools. Nonetheless it is simply possible to group the pixels of the image whose the result is a partition. Such a significant image already segmented represents mainly a set of segments that collectively cover the entire image. Mimics allows the user to create models based on the gray values (Hounsfield units in CT images) within these images. There is a direct association between material density of the scanned object and the gray value assigned to each pixel in the image data [27] and because of this, Mimics has the flexibility to create models from any geometry distinguishable within the scanned data. Many of the segmentation tools in Mimics are common in image processing and can be applied in any of views or in 3D set acting with 3D editing tools. Figure 24: Implant, cortical and trabecular bone The CT images transferred to Mimics software are obviously focused on the proximal part of the femur, on the top of the bone, where the acetabular section has been taken off to avoid the segmentation of the pelvis zone as well. Femur is segmented from the other tissue with pointbased and region-based methods. The point-based method uses only the attributes of an image element for its segmentation. With region-based methods, image elements are attached to segments in the context of their neighborhood. Thresholding is a point-based approach to image segmentation and was the technique adopted and suited for the expected result. Threshold values for the shaft of femur are chosen 226-2500 HU for distal and proximal femur. These parameters were determined from image analysis in order to point out bones features. The HU values for the ends of femur turned out to be a lot lower than for the shaft. Finally, the segmentation is improved manually. Even though mimics tools are reliable, the manual phase has always been foreseen to get accurate masks showing fewer artifacts. In Figure 24 final structures segmented have been shown, pointing out implant features and distinguishing trabecular and cortical bone. Next the head and neck of the operated femur have been cut off roughly at the same height of the real cut made by the surgeons (see Figure 25a). During the elaboration, it is indeed considered the postop image in order to reply the position of the implant fitted in the bone. The more accurate this phase has been held the more accurate forces analysis will be gotten. It is supposed to change the type of cut depending on the implant features but actually this hypothesis is not always respected, thus we could need to cut with different forms and dimensions of the cutting planes always responding to the real surgeons work. The model has been split in two parts and the implant already obtained was fitted
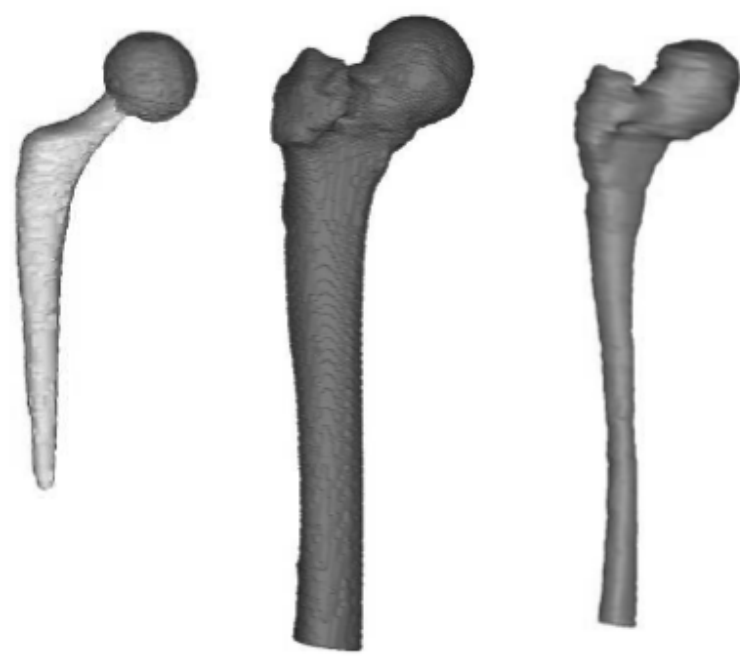

(a) Head cut

(b) Op.Femur

and CT masks

Fig 25 Segmentation results

inside the trabecular bone, avoiding cortical bones overlapping. The cortical bone indeed should not touch the stem at any place according to what happens in the stem fitting made by the surgeons during the operations in hospital. Attention has to be paid: a model that shows an implant nearby the cortical bone can be held in consideration unless the prosthesis material is not definitely in contact with the cortical part. The device edges can swing in the trabecular part of the femur and not in the cortical, and this is what happens when surgeons fix it. Hence the model has to mimic this situation otherwise completely wrong results in fraction risk analysis might be obtained. Five masks have been built in the bidimensional environment of mimics, as the picture 25b reports from a Mimim screenshot in two dimensions. The 2d segments should be exactly complementary each other and avoid pixels holes. The single segment mainly becomes the base on which the software, considering the slices, builds the three dimensional segment. According to studies in materials and their properties, every 3d structure has been associated to material coefficients in order to reply the real mechanical behavior once the forces have been applied: Trabecular femur is the mask that surrounds all the inner part of the bone. During the modeling phase has been carefully analyzed to prevent eventual holes, it has in each part to be deep enough in order to avoid imperfections as the subsequent phases realize a corrosion of the mask. Cortical femur has been shown in the image as the intermediate medium separating implant and cortical bone. On the top, the $2 \mathrm{~d}$ structure of trabecular bone has been cut to reply a real rasp action that surgeons accomplish to fit the implant in the 
zone between great trochanter and femur neck. Prosthesis stem represents the stem part of the implant. Once the material has been introduced and fitted, the most important aspect of the work is to realize a mask strictly adjoined the surrounding trabecular femur, never to the cortical neighbor. Polyethylene liner and titanium cup overhang the stem part on which applies the hip joint load. Attention on making both the masks exactly complementary.

\subsubsection{Meshing}

Many kinds of meshing are related to solid FEA analysis. Experts aim a systematic analysis and evaluation of algorithms for automatic meshing from solid models. Focusing on algorithms that can be integrated into existing solid modeling-based CAD systems, only three families of algorithms have been included: element extraction, domain triangulation, and recursive spatial decomposition. Algorithms in these families are particularly important because first, they tend to satisfy more closely the conditions for genuine automatic operations, second, they have been shown to be applicable to 3D (solid) domains, and third, they are well documented [11]. Mimics has been designed to remesh 3D objects (STL files) from their original RPready format to CAE ready format. To optimize the mesh and create equilateral triangles, it will analyze the shape quality of each triangle. After the quality of each one within a mesh is known it is possible in an automatic way to perform the remeshing processes described through the commands of mimics. Obviously the manual edit is possible and often is the best way to optimize the object for the subsequent finite element analysis. There is not an only one way to mesh the model, actually to optimize the result the established protocol is always adapted to the model features (usually depends on the quality of CT images) selecting different parameters and improve the model. So the target is to obtain a meshed object presenting triangles connected each other. If the volumes are messed up by connecting wrong triangles or by discontinuity, those particular volumes might not appear when the volume mesh is created. The number of triangle nodes and volume elements cannot exceed the maximum allowable number of nodes in Ansys. It seems that magnitude of about 6,000 and 20,000, respectively are the minimum and the maximum.

\subsubsection{Assigning materials properties:}

Mechanic of materials: Subject-specific finite element analysis (FEA) of the skeleton, a very powerful tool for

$$
\begin{gathered}
\rho_{\text {app }}=\frac{\text { hydrated tissue mass }}{\text { total specimen volume }}\left[\frac{\mathrm{g}}{\mathrm{cm}^{2}}\right] \\
\rho_{\text {ash }}=\frac{\text { ash mass }}{\text { total specimen volume }}= \\
=\rho_{\text {volume }}-\rho_{\text {water }}\left[\frac{\mathrm{g}}{\mathrm{cm}^{2}}\right]
\end{gathered}
$$

biomechanical research, is now being adopted in clinical applications. The most common way to create the subject-specific FE models is by deriving information from $\mathrm{X}$ ray computed tomography (CT)

$$
E=\frac{\text { tensile stress }}{\text { tensile strain }}=\frac{\sigma}{\varepsilon} \quad[P a]
$$

images. Information on the mechanical properties of bones can be indeed derived from CT-data, using mathematical relationships between the CT values and mechanical properties of the bone [27]. A large number of mathematical relationships between densitometric measures and mechanical properties have been adopted but in most cases with no explicit considerations on the suitability and accuracy of the chosen one. In order to obtain this correspondence we need of three main mechanic parameters and to review literature to adopt accurate parameters for our study: Apparent density and ash density: the DEXA (Dual Energy X ray Absorptiometry) technique analyzes the attenuation of $\mathrm{X}$-rays as they pass through an area of the body [31]. The method cannot detect the depth of the bone which is being measured, and thus is actually an "areal" density in $\mathrm{g} / \mathrm{cm} 2$ rather than a "volumetric" or Archimedean density in $\mathrm{g} / \mathrm{cm} 3$. As bones grow, the volume increases at a faster rate than the area, so the real bone density will increase even if the volumetric density remains stable [12]. There are different valors on literature to reach a final value of BMAD. What we used is subsequently reported according to the CT protocol adopted in the hospital and to the literature (total specimen mass: the specimen mass including marrow). The ash density is actually the one we insert in mimics in order to take advantage of this during the strain test in Ansys.

Young's Modulus: is defined as a coefficient of elasticity of a substance, expressing the ratio between a stress that acts to change the length of a body and the fractional change in length caused by this force:

For a force tending to elongate a specimen, the tensile stress is the ratio of the force (F) applied in a particular direction in the specimen to the cross-sectional area (A) of the specimen in a plane normal to the direction of the applied force.

$$
\sigma=\frac{F}{A} \quad\left[\frac{N}{m^{2}}\right]
$$

If an increment in the tensile stress applied to a specimen of length (l) produces an increment in length (dl) parallel to the direction of application of the stress, the increment in tensile strain is given by the next relation and has to be integrated on the force direction (given that is monodimensional): 
Total hip replacement: structures modeling, gait analysis and report

European Journal Translational Myology - Basic Applied Myology 2012; 22 (1\&2): 69-121

$$
\partial \varepsilon=\frac{\partial l}{l} \quad \varepsilon=\int_{l_{0}}^{l_{1}} \frac{d l}{l} \quad \begin{aligned}
& \text { Poisson ratio: } \\
& \text { An important } \\
& \text { material }
\end{aligned}
$$

property used in elastic analysis and needed for our

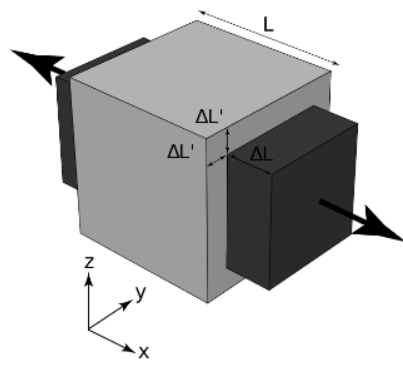

Fig 26 Poisson cube

Ansys analysis is the Poisson's ratio.

It is defined as the ratio of transverse to longitudinal strains of a loaded specimen. In realistic terms, Poisson's ratio can vary from initially 0 to about 0.5 (assuming no specimen volume change after loading). Generally, "stiffer" materials will have lower Poisson's ratios than "softer" materials [13]. We might see Poisson's ratios larger than 0.5 reported in the literature; however, this implies that the material was stressed to cracking, experimental error, etc (Figure 26).

$$
v=-\frac{d \varepsilon_{\text {trans }}}{d \varepsilon_{\text {axial }}}=-\frac{d \varepsilon_{y}}{d \varepsilon_{x}}=-\frac{d \varepsilon_{z}}{d \varepsilon_{x}}
$$

Method: It is common knowledge that the HU acquisition scale changes from time to time and depending on the different kilovoltage values for the tube supply. This variation is less sensible when the voltage used is $120 \mathrm{KV}$. Results in literature suggest the use of customized $\mathrm{HU}$ intervals for studying bone and muscle density changes. These intervals must take into account the type of CT scan device, the used protocol, and the $\mathrm{HU}$ variability which is greater for materials at the extremes of the HU scale [22]. Thus the CT scan used with enough accuracy is CT Scan Philips Brilliance $120 \mathrm{KV} 64$ slices 0.5mm slice increment $1 \mathrm{~mm}$ slices thickness. All the relations are obtained considering this setup. We considered a new summarized study [27] for implant material properties that involves previous articles on the matters in order to figure out with which parameters it is supposed to realize an accurate study, the other main sources are used to calculate for both trabecular and cortical bone mechanical factors. The relationship between ash density of bone and Hounsfield units [38]:

$$
\rho_{a s h}=0,02436+0,0004 H U \quad\left[\frac{g}{\mathrm{~cm}^{2}}\right]
$$

Hence it is possible to calculate the Young's modulus through the ash BMD:

$$
E[M P a]=10500 * \rho_{\text {ash }}^{2,29}
$$

These equations can be used to represent both the soft bone (trabecular/sponge bone) and the compact/cortical bone. The correspondent values for titanium implants and associated elements are from empirical testing. As an example, an uncemented stem made of CLS/PROTASUL 100(Ti6AL7NB) has a density of $4.5 \mathrm{~g} / \mathrm{cm} 3$, Young's modulus (E) of 110000 $\mathrm{MPa}$, and Poisson ratio of 0.3 [5]. The metal cup [32], bone cement, and polyethylene liner properties can be found in similar literature utilized for bone.

\subsection{Analyzing}

\subsubsection{Theory of FEA process}

This section discusses material relationships for linear materials (Figure 27). The stress is related to the strain by:

$$
\sigma=[D] \varepsilon^{e l}
$$

where:

$\sigma=$ stress vector $=\left[\sigma_{x} \sigma_{y} \sigma_{z} \sigma_{x y} \sigma_{y z} \sigma_{z x}\right]^{T}$

$\mathbf{D}=$ elasticity or elastic stiffness matrix or stress-strain matrix or, for a few anisotropic elements, defined by full matrix definition

$$
\varepsilon^{e l}=\varepsilon-\varepsilon^{\text {th }}
$$

strain vector, strains that cause stresses.

$\varepsilon=$ total strain vector $=\left[\varepsilon_{x} \varepsilon_{y} \varepsilon_{z} \varepsilon_{x y} \varepsilon_{y z} \varepsilon_{z x}\right]^{I}$

$\varepsilon^{\text {th }}=$ thermal strain vector (this part is not deepened in the discussion) The stress vector is shown in the figure below. The sign convention for direct stresses and strains used throughout the ANSYS program is that tension is positive and compression is negative. For shears, positive is when the two applicable positive axes rotate toward each other.
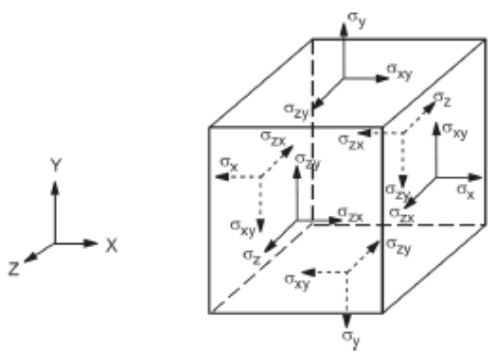

Fig 27 Stress vector definition 
The flexibility or compliance matrix, [D]-1 is:

$$
\left[\begin{array}{cccccc}
1 / E_{x} & -v_{x y} / E_{x y} & -v_{x z} / E_{x} & 0 & 0 & 0 \\
-v_{y x} / E_{y} & 1 / E_{y} & -v_{y z} / E_{y} & 0 & 0 & 0 \\
-v_{z x} / E_{z} & -v_{z y} / E_{z} & 1 / E_{z} & 0 & 0 & 0 \\
0 & 0 & 0 & G_{x y}^{-1} & 0 & 0 \\
0 & 0 & 0 & 0 & G_{y z}^{-1} & 0 \\
0 & 0 & 0 & 0 & 0 & G_{x z}^{-1}
\end{array}\right]
$$

$E_{x}=$ Young's modulus in the $\mathrm{x}$ direction

$$
\begin{aligned}
& v_{x y}=\text { Major Poisson's Ratio } \\
& v_{y x}=\text { Minor Poisson's Ratio } \\
& G_{x y}=\text { Shear modulus in the xy plane }
\end{aligned}
$$

Because of the above matrix and his property, $\mathrm{n}$ terms are not independent quantities and therefore these values have to be inserted; given the hypothesis of orthotropic material, it is enough to inquire of the source of the material property data as to which type of input is appropriate. This means that correct values of the shear are entered, as there are no defaults provided by the program. Moreover, the matrix [D] has to be definite. The program checks each material property as used by each active element type to ensure that [D] is indeed positive definite. The matrix in observation is related to other factor concerning any thermal excursions affecting on the materials, part of the work which is not foreseen due to absence of thermal significant changes. Another way to influence the matrix is by the effect of consistent pressure loading and surface stresses. Output of the process is a matrix of forces and moments applied to each one of finite element, whose the entire model is composed by. The macro vision allows considering relations between the elements and obtaining an overall prospect of forces and moments. Hence, it is possible to evaluate deformations and movements of the material, considering resultants of forces, moments and going back to the deformations.

\subsubsection{Strain test}

Structural failure becomes increasingly likely as the load on bone approximates or exceeds the bone's ability to withstand it. The bone fracture risk index

Table 3 Values of reference for strain rate

\begin{tabular}{|c|r|}
\hline Strain rate (1/s) & Yield strain (\%) \\
\hline 20 & 0,5 \\
\hline 15 & 0,6 \\
\hline 10 & 0,7 \\
\hline 5 & 0,8 \\
\hline 0 & 0,9 \\
\hline
\end{tabular}

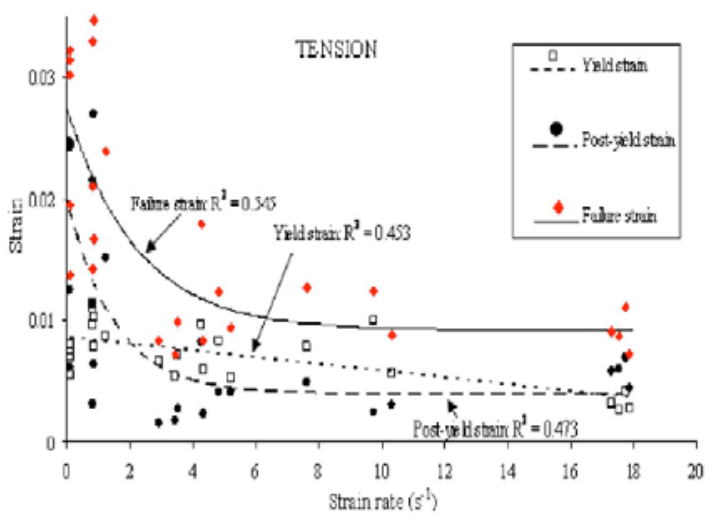

Fig 28 Tension graph

(FRI) expresses the risk for structural failure as a ratio of compressive stress (load per unit area) to estimated failure stress, and so should be a more sensitive and specific predictor of fracture considered together with BMD observations. Despite real consistency and response of bone, it is supposed to manifest an isotropic demeanor with a Poisson's ratio equal to 0.3. Yield strain has not been correlated thoroughly to apparent density in the literature. However, according to [2] indications exist to suggest that yield strain is relatively independent of apparent density and is often reported in the range of $0.6 \%$ to $1.2 \%$. A value of \#y,b $=0.9 \%$ was assumed to represent a catastrophic failure of bone in the present study. Failure at the interface, in this case, was assumed to be present if equivalent Von Mises strains exceeded the yeld strain\%. The results are in form of Von Mises equivalent stress and the fracture risk index is calculated with the following equation:

$$
\text { FRI } \%=\frac{\varepsilon_{\max }}{\varepsilon_{\text {yield }}} * 100 \%
$$

Where epsilon max is the value of the strain at a given point and \#yield is the yield strain value which is considered as a catastrophic failure of the bone. In future developments of the project we will consider

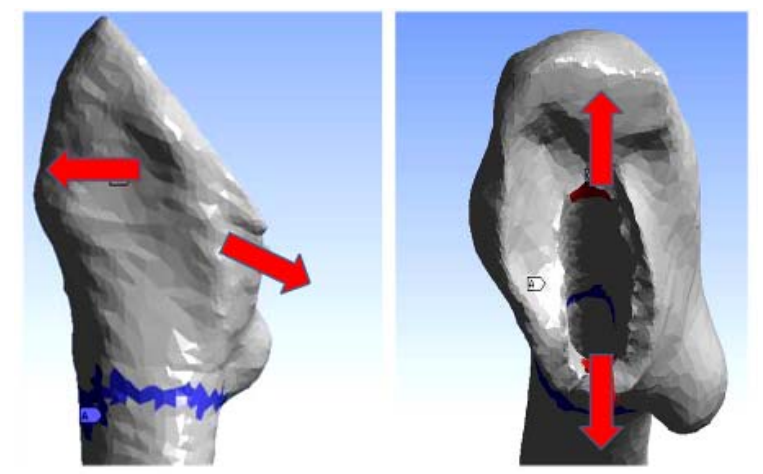

Fig 29 Ansys boundaries conditions 


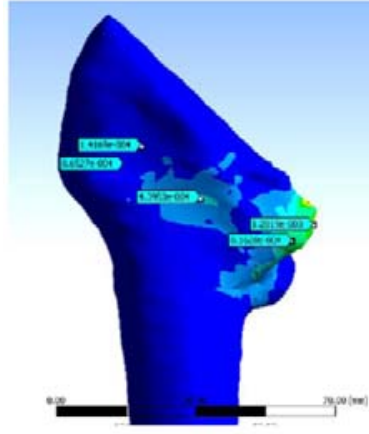

(a) Ex 1

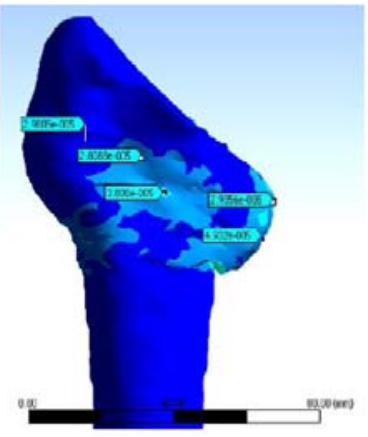

(b) Ex 2
Fig 30 Strain test

Yield Strain as a parameter dependent on strain rate as the graph from Figure 28 has depicted (taken from literature), and used to calculate the yield strain for each strain value [26], where already done tries resulting in scatter point have been distinguished in red and black to underline combinations of setups leading failure consequence. Therefore the based on Table 3 can be held in consideration to gather refer numbers.

Boundary conditions for the strain test were selected as the Figure 29 demonstrates. The analysis is briefly focused on evaluating fracture risk around the stem insertion hole where the press fitting is relatively close to be unbearable for cortical edges, and for this reason a mandatory fixed support which the entire bone stands on is right below the minor trochanter to bear subsequent movements. The forces have been applied in correspondence of the stem at the points where the shape is the thickest and the highest force can be expected, and used to simulate the elongation of the elastic bone tissue when the stem is pushed into the femur.

Strains applied on the shape are usually $1000 \mathrm{~N}$ in each direction, not retrieved from any literature, and should take into account the prospect to mimic hammering of the surgeon during the surgery that can be roughly 30 to 50 hits. Notwithstanding due to the fact that we could not simulate the hitting, expecting there will be likely some additive/cumulative effect of the force, stronger strain was selected and applied supposing that the force usually applied in one strike with the hammer tool would cover the cumulative strain. Meanwhile the calculation of the fraction risk index has been accomplished, the strain rate has been taken into account, because of the short duration of the force applied from the surgeon representing further information likely meaningful for surgeons. The effective strain rate in an element is dependent not only on the rate of loading but also such factors as the shape and dimensions of the specimen, therefore a meaningful parameter that consider the deformation per second to determine in how long time a certain effect happened has been included in.

Instead, and actually is what we consider, it is used to have deformation after a certain period o $\mathrm{f}$ time: in order to get the strain rate (strain/time), the strain has been divided for the duration of the strike, which is very short around 0,10 ms. Results of the strain test analysis made trough Ansys tools assume the form of images where colors ( indeed a scale of tonalities as been used by the software, from blue to red color in crescent quantity of strain) mean quantity of yield strain, or rather equivalent Von Mises elastic strain, applied on bone shape in order to show the areas on which a stronger effort impresses. On this view of the structure of the femur, usually 5 zones have been pointed out in order to figure out correspondent values of FRI and strain rate subsequently reported in tables. Taking a look on the pictures derived from the software in Figure 30, colors on the shape surface underline mainly the major zones where a fracture during the operation can be caused, roughly across the intertrochanteric zone and especially on the brinks beneath the cut for the stem fitting. In general the most interested zones for intra-operative failure, as common surgeon's knowledge and analysis argued, follow the intertrochanteric line, starting from the proximal femur middle level and ending up proper the minor trochanter jut. The first case denounces major stress on brink where implant has been situated up the minor trochanter. For the second example minor probability of failure during fitting pressure has been shown in because of morphology of the impact zone and quantity of stress on the cortical bone evidently less porous. Certain conclusions derived by color layout are

\begin{tabular}{|c|c|}
\hline Strain rate (1/s) & FRI\% (\%) \\
\hline \hline 0,000142 & 1,610114 \\
\hline 0,000865 & 12,01764 \\
\hline 0,00044 & 5,494125 \\
\hline 0,001282 & 18,85147 \\
\hline 0,000816 & 11,33722 \\
\hline
\end{tabular}

\begin{tabular}{|c|c|}
\hline Strain rate (1/s) & FRI\% (\%) \\
\hline \hline 0,000029805 & 0,3311667 \\
\hline 0,00028088 & 0,3120889 \\
\hline 0,00003808 & 0,4231111 \\
\hline 0,000029256 & 0,3250667 \\
\hline 0,00004932 & 0,548 \\
\hline
\end{tabular}

Table 4 e.g. FRI and Strain Rate 

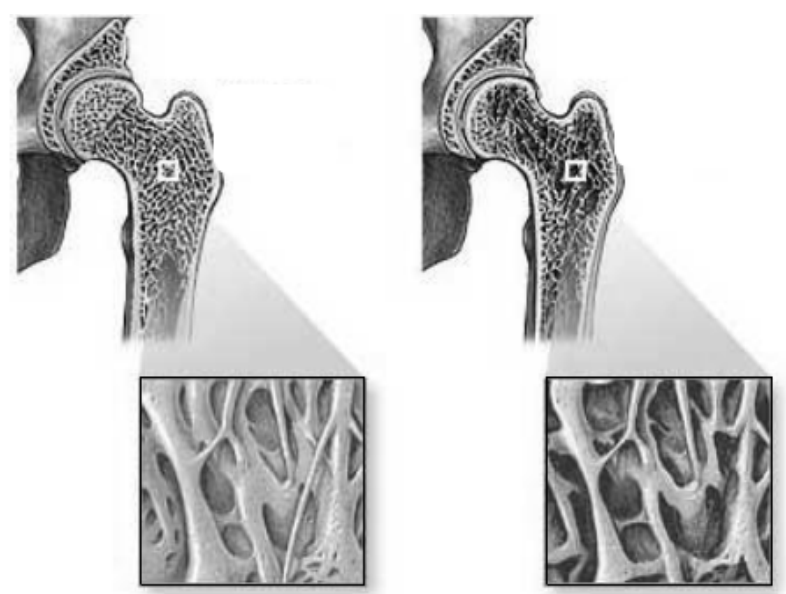

Fig 31 Differences in osteoporotic BMD

further argued trough FRI tables reported in 4: the first case percentage values of FRI are much higher than the second femur implying major probability of failure in those particular extrapolated areas. Concerning the strain rate, values represent further information that match already given conclusions since time which is necessary in general in the second case to achieve significant deformations is definitely shorter. During the simulation it is supposed to obtain the reaction of the bone meanwhile forces are applied, Ansys allows to record a clip showing resultant stress in each part of the model in movement because of solicitations, thus it is possible to register movies and store them for subsequent works.

\subsection{Bone mineral density measurement}

\subsubsection{Theory of the BMD}

Fractures of the hip, vertebral body, and distal forearm have long been regarded as the typical osteoporotic fractures. Between 220,000 and 250,000 proximal femoral fractures occur in the United States each year; $90 \%$ of these fractures occur in patients older than 50 years. In younger patients, proximal femoral fractures are usually the result of high-energy physical trauma (e.g., high-speed motor vehicle accidents) and usually occur in the absence of disease. Intertrochanteric and femoral neck fractures account for $90 \%$ of the proximal femoral fractures occurring in elderly patients. Proximal femoral fractures in elderly patients are often pathologic, usually resulting from minimal to-moderate physical trauma to areas of bone significantly affected by osteoporosis. However, pathologic fractures can occur at any age [36]; typically, these fractures result from low-energy injuries and may be characterized by unusual fracture patterns. The incidence of proximal femoral fractures among females is 2 to 3 times higher than the incidence of such fractures among males. Also, the risk of sustaining a proximal femoral fracture doubles every 10 years after age 50 years. Other risk factors for

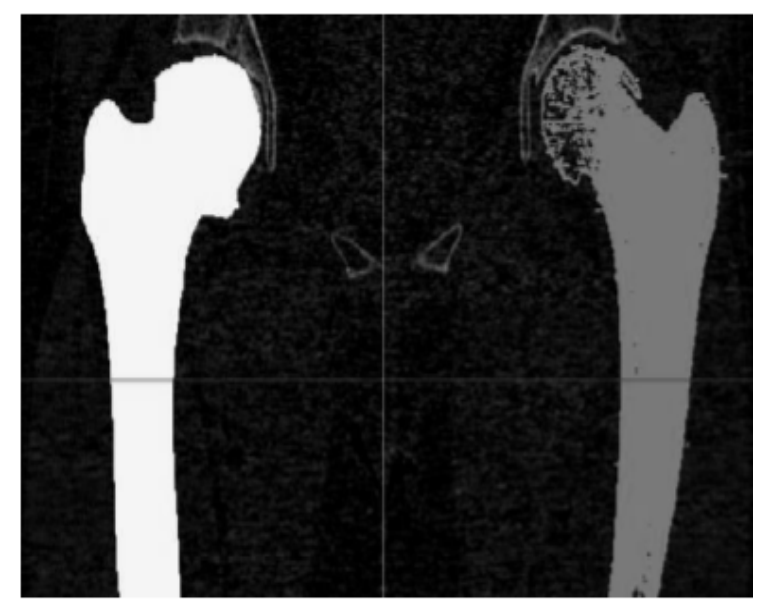

Fig 32 Masks of femurs

proximal femoral fractures include osteoporosis, a maternal history of hip fractures, excessive alcohol consumption, high caffeine intake, physical inactivity, low body weight, previous hip fracture, the use of certain psychotropic medications and smoking, the fracture has moreover a higher incidence in women than men. Despite the wide prospect of fractures or pathologies afferent the hip joint, it is generally considered that these differences in fracture incidence or diseases reflect, in part, higher bone strength in men than women, and in blacks than whites (studies from America) according to diverse bone structures in density conformation. Two major components of bone strength at the hip are BMD and bone geometry (utilized in modeling). Both femoral bone density [36] and hip geometry predict hip fracture. Bone density is the primary factor contributing to bone strength, accounting for $60-70 \%$ of the variance in ultimate strength of bone tissue [40] (Figure 31). A loss of bone density affects overall bone strength and increase the risk of fracture. Therefore knowledge about bone density and its distribution in femur for the patients gives valuable information concerning strength. Bone density is expressed as grams of mineral per volume.

BMD can give a good indication of bone quality and its change with time [41]. Using CT scan and our softwares, values of BMD are available, rapidly and easy done, and reliable in noninvasively determining BMD (Figure 32). Although many studies have evaluated the BMD after total joint arthroplasty [41], most of them mainly considered periprosthetic BMD to check loosening of the components [40]. In this section a method to evaluate it is reported in order to obtain BMD for healthy and operated femurs in patients. We compare them before the operation and further one year later to control the bone growth and stability. Given that in literature most of the studies are focused on BMD Age- Race correlations, our goal is to underline relations between BMD and implants applied to the femur, aiming a decisional support for surgeons 


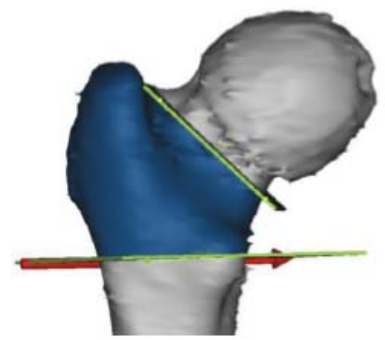

(a)

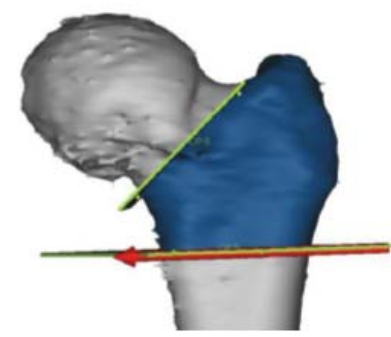

(b)

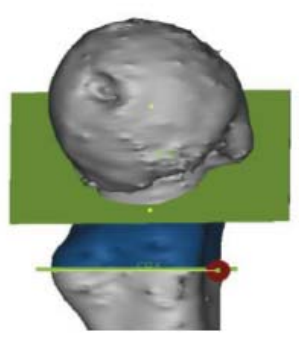

(c)

Fig 33 Views of the ROI

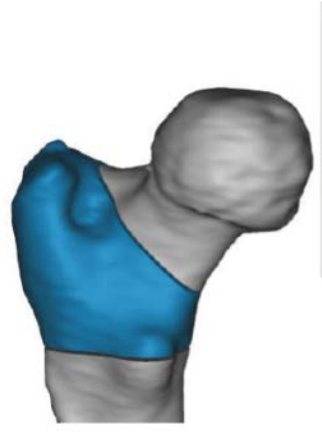

(a)

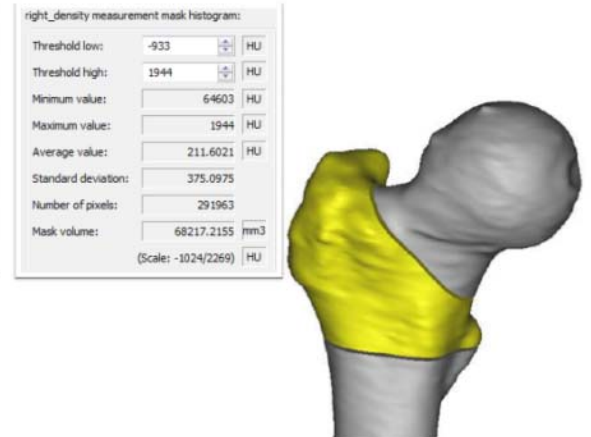

(b)

Fig $34 B M D$ Too

concerning different choices. Otherwise, if no relevant statistics are obtained hence it is supposed to discover absence of meaningful connections between prosthesis fixed and preoperative BMD measurements. The hypothesis is that patients with low BMD have to be treated by cemented implant, otherwise the femur is strong enough to stand an uncemented implant with all the advantages of the case. As already mentioned, the most common fracture sites of femur are femoral neck and trochanter, therefore the BMD of proximal femur is researched more carefully.

\subsubsection{Method}

Same CT slices used by previous modeling part for Ansys now have been taken back in consideration. Femur is segmented (therefore it is distinguished) from other tissues with point method and region-based method of pixel grouping. The point-based method uses only the attributes of an image element for its segmentation. With region-based methods, image elements are attached to segments in the context of their neighborhood. Thresholding is a point-based approach to image segmentation and was the technique of choice for this project.

Threshold values for the shaft of femur are chosen 226-2500 HU for distal and proximal femur. These parameters were determined through image analysis in order to point out bones features. HU values for the ends of femur turned out to be a lot lower than for the shaft. Masks of healthy and operated femurs are created always through mimics tools and are not cave in order to consider the internal density as well (refer to Figure 33). Nevertheless the bone is diverse in cortical part, sponge bone or proximal and distal shapes, a unique constructed mask has been realized. The aim of this phase is indeed to get an overall prospect on density as much as possible similar considering left and right femur. Corresponding 3d model has been calculated and smoothed because of the artifacts with an adapted number of smooth mimics interactions (9-15 depending on the quality of the model). Only the ROI has to be considered in the femurs, these are obtained by polyplanes cutting that mimics dispenses. These incisions have been done taking advantage from measurements of distance by ruler and goniometers provided by the software. Therefore points of cut are individuated accurately and in an objective way on the bone shape. Notwithstanding this guide tool, actually the most challenging part of the work is still referring measurements to similar ROIs both for operated and healthy side. Usually two cuts have been done on the shape of proximal femurs, respecting volumes studied and bone conformation. First cut begins from the greater trochanter and two centimeters above the minor trochanter it ends. The top of the femur trochanter zone is directed a little lateralward and backward, and, in the adult, is about $1 \mathrm{~cm}$ deep in cortical bone. Since the upper part is a large, irregular, quadrilateral eminence, situated at the junction of the neck with the upper part of the body, the cut edge follows obturator internus and gemelli pursuing toward the articular capsule across 
Total hip replacement: structures modeling, gait analysis and report

European Journal Translational Myology - Basic Applied Myology 2012; 22 (1\&2): 69-121

\begin{tabular}{|c|c|c|c|c|c|c|c|}
\hline \multicolumn{8}{|c|}{ CEMENTED } \\
\hline Age & Gender & Weight & Operated side & BMD Op.[HU] & BMD Hea. [HU] & BMD Op. [mg/cm^3] & BMD Hea. $\left[\mathrm{mg} / \mathrm{cm}^{\wedge} 3\right]$ \\
\hline 56 & $\mathrm{~F}$ & 76 & Left & 330 & 345 & 156,36 & 162,36 \\
\hline 58 & $M$ & 74 & Left & 234 & 307 & 117,96 & 147,16 \\
\hline 66 & $\mathrm{~F}$ & 66 & Right & 345 & 342 & 162,36 & 161,16 \\
\hline 67 & $\mathrm{~F}$ & 70 & Right & 241 & 275 & 120,76 & 134,36 \\
\hline 67 & $\mathrm{~F}$ & 80 & Right & 351 & 361 & 164,76 & 168,76 \\
\hline 68 & $\mathrm{~F}$ & 78 & Right & 249 & 274 & 123,96 & 133,96 \\
\hline 71 & $\mathrm{~F}$ & 95 & Left & 277 & 283 & 135,16 & 137,56 \\
\hline 73 & $\mathrm{~F}$ & 69 & Right & 228 & 239 & 115,56 & 119,96 \\
\hline 75 & $\mathrm{~F}$ & 63 & Right & 271 & 282 & 132,76 & 137,16 \\
\hline 77 & $\mathrm{~F}$ & 80 & Right & 213 & 237 & 109,56 & 119,16 \\
\hline \multicolumn{8}{|c|}{ UNCEMENTED } \\
\hline Age & Gender & Weight & Operated side & BMD Op.[HU] & BMD Hea.[HU] & BMD Op. $\left[\mathrm{mg} / \mathrm{cm}^{\wedge} 3\right]$ & BMD Hea. $\left[\mathrm{mg} / \mathrm{cm}^{\wedge} 3\right]$ \\
\hline 21 & $\mathrm{M}$ & 66 & Right & 432 & 491 & 197,16 & 220,76 \\
\hline 43 & M & 87 & Left & 379 & 413 & 175,96 & 189,56 \\
\hline 46 & $\mathrm{M}$ & 100 & Right & 347 & 403 & 163,16 & 185,56 \\
\hline 52 & M & 95 & Left & 370 & 396 & 172,36 & 182,76 \\
\hline 53 & $\mathrm{M}$ & 60 & Left & 278 & 357 & 135,56 & 167,16 \\
\hline 56 & $\mathrm{~F}$ & 83 & Right & 287 & 309 & 139,16 & 147,96 \\
\hline 59 & $\mathrm{~F}$ & & Right & 246 & 301 & 122,76 & 144,76 \\
\hline 63 & $\mathrm{M}$ & 85 & Left & 291 & 301 & 140,76 & 144,76 \\
\hline 63 & $\mathrm{~F}$ & 96 & Left & 211 & 212 & 108,76 & 109,16 \\
\hline 68 & $\mathrm{~F}$ & 80 & Right & 227 & 258 & 115,16 & 127,56 \\
\hline
\end{tabular}

Table 5 BMD Results

the intertrochanteric line (please refer to reported Figure 33a-c in order to manage a better comprehension). Running obliquely downward and medialward from the tubercle is this spiral line of the femur and the cut indeed profiles it. Its upper half is rough, and affords attachment to the iliofemoral ligament of the hip-joint; its lower half is less prominent, and gives origin to the upper part of the Vastus medialis. As already said, the incision leads above the minor trochanter, roughly two centimeters upper. The second horizontal incision has been done in the middle of the lesser trochanter, which is a conical eminence varying in size depending on subjects; it projects from the lower and back part of the base of the neck. Following images display ROIs and cuts applied as well as described previously. It is possible to notice that the section of interest contains exactly so-called greater trochanteric zone and intertrochanteric zone, depicted in Figure 34 a-b. ROIs have a form elaborated according to surgeons' reports and literature concerning BMD evaluations on femur structures. Proceeding to split all the parts of the model once cuts have been done and colored in gray besides the ROI which has to be underlined by light aspect. Finally the mask of the ROI model has been calculated and using properties view of Mimics it is possible to obtain several parameters regarding the proximal femur among which is reported the average value of bone density in HU. To determine the relation between Hounsfield units and BMD values, Hounsfield units have to be measured on phantoms. The adopted calibration [23] leads to a linear relationship: BMD = $a * H U+b$ b, where a and b are calibration coefficients, which were calculated from the phantom data along with the corresponding statistical descriptors. Voxel values of the preoperative scans concerning healthy and operated femur were converted from Hounsfield

$$
B \mid M D\left[\frac{m g}{c^{3}}\right]=0,40 H U+24,36
$$

The relation respects modality of acquisition for the scans: (64 CT) Philips Brilliance was the machine utilized to $120 \mathrm{KeV}$ with a correlation of R2 almost 0.99 .

\subsubsection{Results}

Results concerning BMD for patients studied whose CT scans were already used is reported in Table 5, where subjects conditions have also been considered. However outcomes of elaboration have been distinguished in cement and cementless implant used as the project expectations were based on. Each one of 

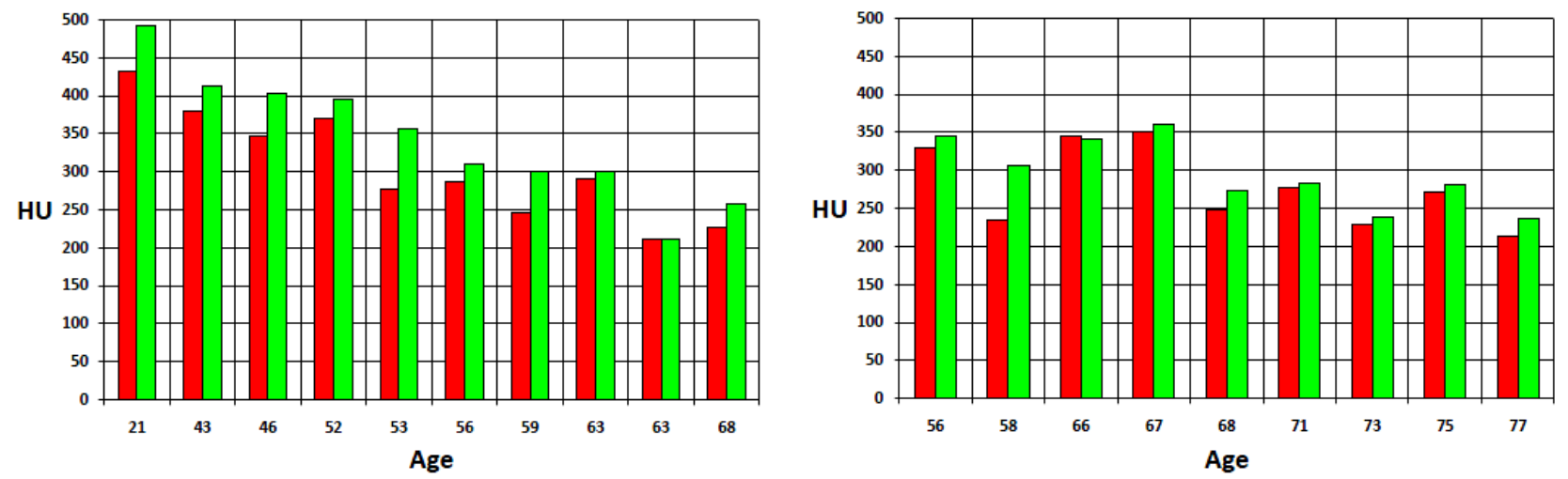

Fig 35 Sample distribution for BMD

the patients is characterized by a BMD value in $\mathrm{g} / \mathrm{cm} 3$ both for healthy and operated side respectively colored in green and red in the charts proposed in Figure 35.

A previous and simple observation can be held. The period of accelerated loss of bone mineral density in the hip bones occurring among women and men older than 65 may be an important contribution to the increased incidence of hip fracture among patients in that age group. The extent of bone loss that we observed in both sexes and series of cemented and uncemented proves the already known strictly connection BMD-Aging, further argued by a real different value for the patient 21 years old in uncemented group. Once again, the aim of this project section is to prove possible connection between surgeons choices and adopted implant. It is supposed to apply uncemented prosthesis to patients showing femurs enough strong to stand loads led by the biocompatible stem without a cemented medium. By charts concerning BMD values in average it is possible to notice, even though an overall vision is considered, that treated patients belonging to lower values in average group underwent cemented replacement. Otherwise cementless group denounces higher amounts. Thus, cement implants are usually suited and adopted for patients that appear in tricky conditions, where is though to let the prosthesis standing on close bones, borne by them, because of frail inner parts. The charts report this demeanor and distinguish however healthy and operated side. Furthermore, measurements and results, in this way shown, confirm the chance to participate in implants choice: surgeons could base their decisions also on the results of previous BMD analysis before the operation done. Hopefully, they might get decision adapted thank to objective parameters rather than $\mathrm{RX}$ observations only.

\section{GAIT ANALYSIS}

A Gait Analysis is an evaluation of the manner or style of walking, usually done by observing the feet as it walks or trots in a straight line. The normal forward step consists of two phases: the stance phase, during which one or more legs and feet are bearing most or all of the body weight, and the swing phase, during which the other feet are not touching the walking surface and the body weight is borne by the others. In a complete two-step cycle all feet are in contact with the ground at the same time for about $25 \%$ of the time. This part of the cycle is called the double-support phase. An analysis of each component of the three phases of ambulation is an essential part of the diagnosis of various neurological disorders and the assessment of patient progress during rehabilitation and recovery from the effects of a neurological disease, a musculoskeletal injury or disease process, or amputation of a lower extremity.

In this chapter will be faced arguments regarding the analysis of the walk in correspondence with different instruments used to collect data. After an initial introduction to the typical lexis, there are documented methods and instruments adopted to get information. One part will be focused on muscles involved in the gait because of the possibility in case to unify information from the gait and from EMG devices. This section is divided in: Gait rite: Measurement devices and software to elaborate the gait of the patient data and obtain objective parameters associated to the walk. Kine Pro for EMG: One part of the project in development regards the possibility to compare movies of gait rite with signal traces in order to associate EMG path of specific muscles to the phases of the walk. 


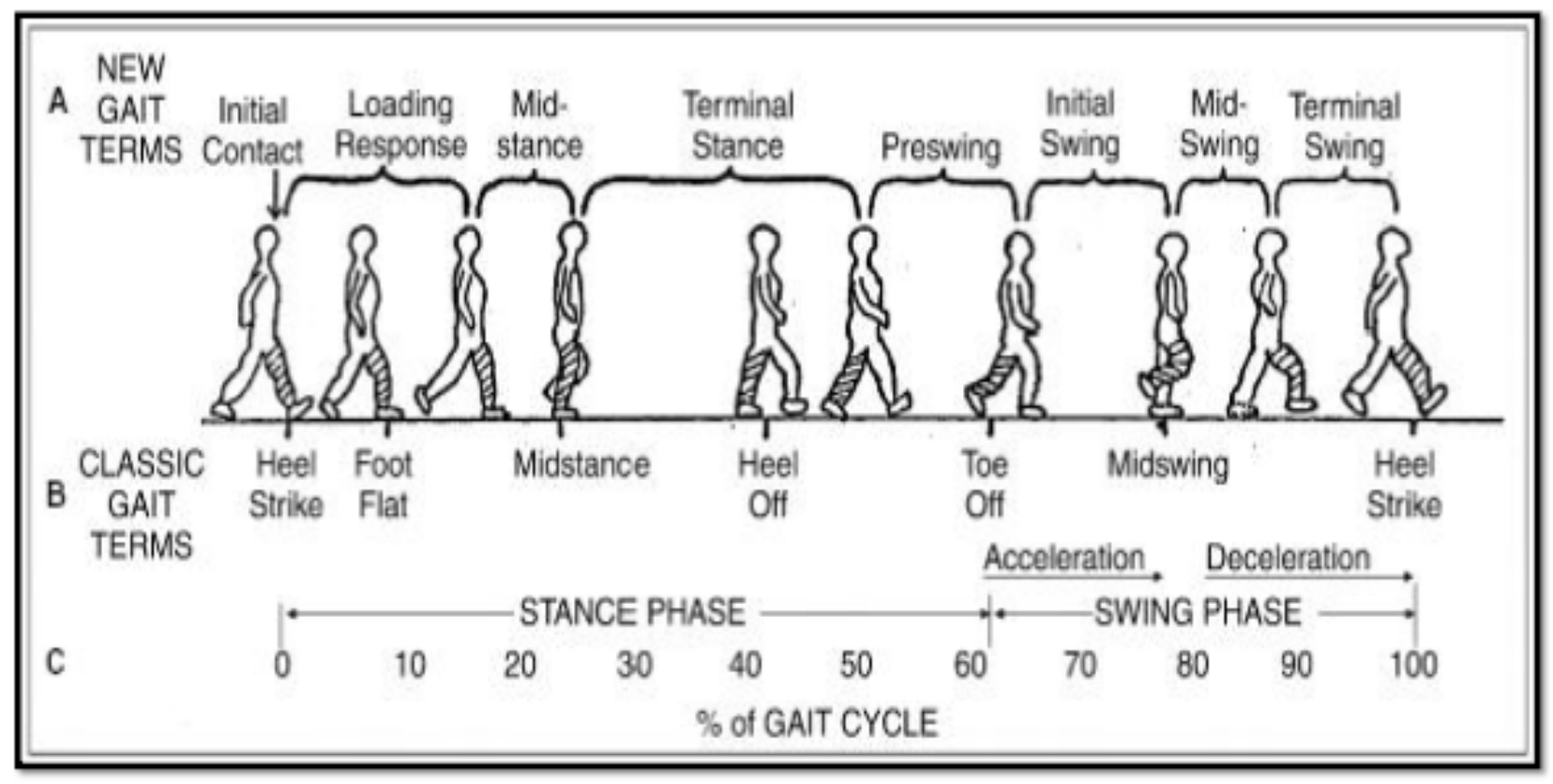

Fig 36 Walk phases

\subsection{The gait}

\subsubsection{Phases}

There are two essential aspects to walking, equilibrium and locomotion (Figure 36). Equilibrium is being able to walk in an upright position and keeping balance. Locomotion is being able to maintain a rhythmic stepping motion. Muscles of the feet and legs are very important as they provide the strength to initiate and maintain movement. The most demanding tasks of the gait cycle are transferring the body weight onto a limb that has just finished swinging. The gait cycle begins when one foot contacts the ground and ends when that foot contacts the ground again. Each cycle begins with the initial contact in the stance phase and moves through a swing phase until the cycle ends with the opposite foot next initial contact. Stance phase accounts for approximately 60 percent, and swing phase for approximately 40 percent of a single gait cycle. Each gait cycle includes two periods when both feet are on the ground. The first period of double foot contact begins at initial contact, and lasts for the first 10 to 12 percent of the cycle. The second period of double foot contact occurs in the final 10 to 12 percent of stance phase. As the stance limb prepares to leave the ground, the opposite limb contacts the ground and accepts the body's weight. The two periods of double foot contact account for 20 to 24 percent of the gait cycle's total duration. Stance phase of gait is divided into four periods: loading response, mid stance, terminal stance, and pre swing. Swing phase is divided into three periods: initial swing, mid swing, and terminal swing. The beginning and ending of each period are defined by specific events.

\section{Period 1: Initial Double Limb Support}

Begins with the first foot strike and ends with the opposite foot's toe-off. The center of mass (COM) begins to rise during this period as the shift to a single limb occurs. It is characterized by rapid loading with shock absorption (mostly from the knee) and slowing the body's forward momentum. The hip begins flexed and then extends as the opposite foot comes off the ground. On the contrary, the knee begins fully extended and then flexes during this period. The ankle goes from a neutral position to plantarflexed until the foot is flat on the floor.

Period 2: Single Limb Support

Begins with the opposite toe off and ends with opposite foot strike. As the body passes over the grounded foot, the COM reaches its peak height while both the forward and vertical velocity decreases. The COM then falls until the end of this period at opposite foot strike, as forward and vertical velocity increase again and forward shear reverses to aft shear. The hip continues to extend throughout this period as the limb progresses from an anterior to a posterior position in relation to the body. The knee extends again until it hits a maximum extension and starts to flex. The ankle dorsiflexes throughout the phase but actively plantarflexes towards the end to resist this dorsiflexion. Period 3: Second Double Limb Support

Begins with opposite foot strike and ends with toe off. The COM is at its lowest level during the beginning portion of this period as both feet are in contact with the ground. The COM then starts to rise again as the 


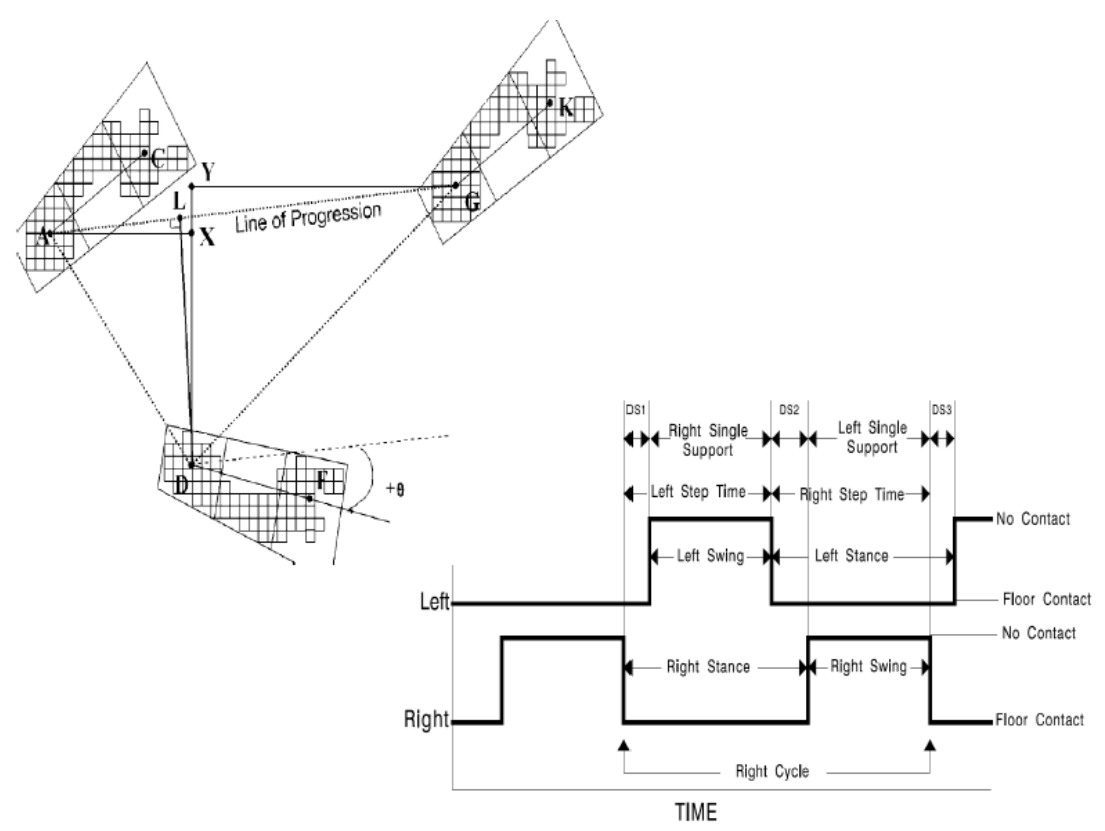

Fig 37 Parameters of walk path

opposite limb is in its initial double limb support. As body weight is transferred to the opposite limb, the trailing limb ends its extension and prepares to swing in front of the body. As a result, the hip and knee flex and the ankle plantarflexes during this time in order to prepare for the limbs lift off.

\section{Period 4: Initial Swing}

It starts the second single limb support interval. It begins when the foot is lifted off the ground (toe-off) and ends when the swinging foot clears the ground and is opposite the standing opposite foot (feet adjacent). The COM reaches its peak height towards the end of this period as the opposite foot is in the middle of single limb support. The ankle continues to plantarflex but then begins to dorsiflex in order to clear the ground. The knee continues to flex rapidly to its peak at the end of this period, which largely results from the hip flexing as well.

\section{Period 5: Mid-Swing}

Middle third of the swing phase. It begins with foot clearance where the feet are adjacent and ends when the swinging foot is in front of the body and the tibia is vertical. The COM falls during this time as the opposite limb is in the middle portion of the single limb support period. The leg's advancement is carried out by continued hip flexion. The knee begins to extend quickly due to momentum and in response to gravity. The ankle continues to dorsiflex to neutral. Period 6: Terminal Swing

Last third of the swing phase. It begins when the tibia is vertical and ends with initial contact(foot strike) of the swinging foot. The COM reaches its lowest level at the end of this period as double limb support begins. The knee continues to extend in order to complete limb advancement. The hip reaches maximum flexion at the beginning of this period and tends to extend slightly before initial double limb support. The ankle remains dorsiflexed to neutral.

\subsubsection{Walk parameters:}

A list of parameters is shown in order to upgrade the knowledge of the writer regarding parameters usually associated to the walk (Figure 37). Given that in the literature there is a wide range of parameters, here we will point to most interesting concerning our observations in the project [7]. Stride Length is measured on the line of progression between the heel points of two consecutive footprints of the same foot (left to left, right to right). (AG) is the stride length of the left foot. The unit of measure is centimeters. Step Length is measured along the length of the walkway, from the heel centre of the current footprint to the heel centre of the previous footprint on the opposite foot. The length of line (AX) is the step length of the right foot, while the length of line (YG) is the step length of the second left foot. The step length can be a negative value if the subject fails to bring the landing foot heel point forward of the stationary foot heel point. The unit of measure is centimeters. It is measured on the line of progression between the heel points of two consecutive footprints of the same foot (left to left, right to right). The unit of measure is centimeters. $\mathrm{H}-\mathrm{H}$ Base of Support or Base Width: It is the vertical distance from heel center of one footprint to the line of progression formed by two footprints of the opposite foot. In Figure, the height of the triangle (ADG) is (DL) which is the base width of the right foot. The unit of measure is centimeters. Toe In / Toe Out: It is the angle between the line of progression and the midline of the footprint. 


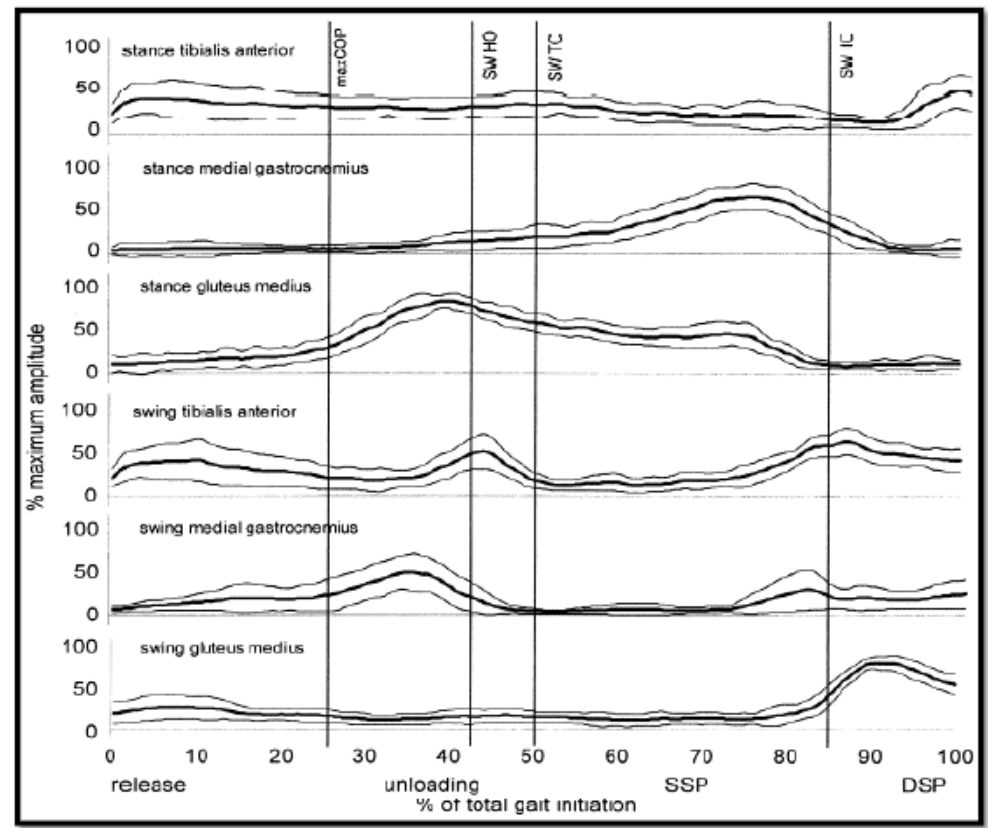

Grand ensemble mean $( \pm 1 S D)$ for all subjects $(n=21)$. For time and amplitude normalised EMG, from swing leg and stance leg muscles during gait initiation. The overall mean times for the major foot contact events for all subjects are shown as vertical lines: maxCOP, the furthest point of initial posterolateral COP movement; SW HO, heel-off of the swing foot; SW TO, toe-off of the swing foot; SW IC, initial contact of the swing foot.

Fig 38 EMGs

In the picture, theta is the angle between the mid-line of the right footprint and the line of progression. An angle theta is zero if the geometric mid-line of the footprint is parallel to the line of progression; positive, toe-out, when the mid-line of the footprint is outside the line of progression and negative, toe-in, when inside the line of progression. The unit of measure is degrees. Single Support and \% Single Support: It is the time elapsed between the Last Contact of the current footfall to the First Contact of the next footfall of the same foot. Single Support time is equal to the Swing Time of the opposite foot. It is measured in seconds (sec) and expressed as a percent of the Gait Cycle time of the same foot. Initial Double Support and \%Initial Double Support: The two periods when both feet are on the floor, are called initial double support and terminal double support. Initial double support occurs from heel contact of one footfall to toe-off of the opposite footfall. It is measured in seconds (sec) and also expressed as a percent of the Gait Cycle time for the same foot. Refer to the figure 37, DS1 is the Initial Double Support for the right foot, while the DS2 is the Initial Double Support for the left foot. Terminal Double Support and \%Terminal Double Support: The two periods when both feet are on the floor, are called initial double support and terminal double support. Terminal double support occurs from opposite footfall heel strike to support footfall toe-off. It is measured in seconds (sec) and also expressed as a percent of the Gait Cycle time for the same foot. Refer to Figure 37,
DS2 is the Terminal Double Support for the right foot. Total Double Support and \%Total Double Support: The two periods when both feet are on the floor, are called initial double support and terminal double support. Initial double support occurs from heel contact of one footfall to toe-off of the opposite footfall. Terminal double support occurs from opposite footfall heel strike to support footfall toe-off. Total double support is the sum of the initial double support added to the terminal double support. It is measured in seconds (sec) and also expressed as a percent of the Gait Cycle time for the same foot. In the picture, the sum (DS1+DS2) is the Total Double Support for the right foot, while the sum (DS2+DS3) is the Total Double Support for the left foot. Stance Time and \% Stance: The stance phase is the weight bearing portion of each gait cycle. It is initiated by heel contact and ends with toe off of the same foot. It is the time elapsed between the First Contact and the Last Contact of two consecutive footfalls on the same foot. It is also presented as a percentage of the Gait Cycle time.

Swing Time and \%Swing: It is initiated with toe off and ends with heel strike. It is the time elapsed between the Last Contact of the current footfall to the First Contact of the next footfall on the same foot. It is expressed in seconds (sec) and it is also presented as a percent of the Gait Cycle of the same foot. The Swing Time is equal to the Single Support time of the opposite foot. 


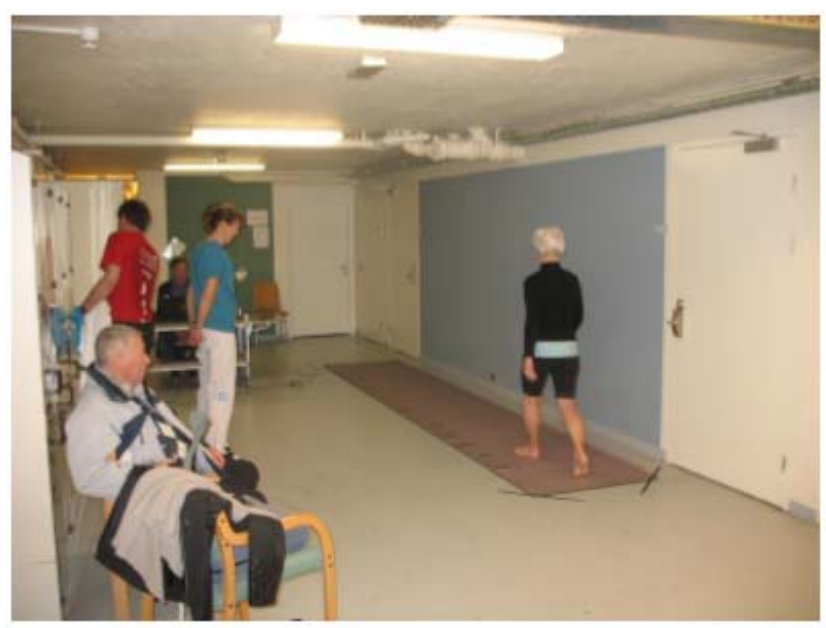

(a)

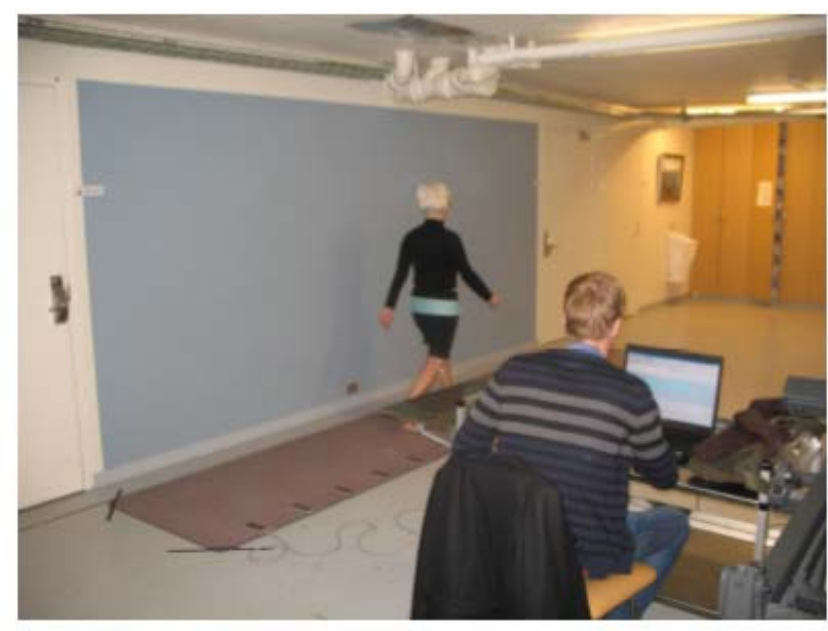

(b)

Fig 39 Grensas rehabilitation center

\subsubsection{Muscles involved}

In the absence of external forces, muscle activity is required to initiate gait from a standstill. An appropriate horizontal ground reaction force (GRF) component must be generated to accelerate the center of mass (COM) forwards and towards the stance side (Figure 38). To achieve this, co-coordinated muscle activity initially moves the center of pressure (COP) poster-laterally towards the swing leg (the first leg to leave the ground) side of the base of support (BOS) [33]: In young adults, the predominant pattern of muscle activity to produce the posterior element of COP movement is a bilateral inhibition of tonic gastrocnemius/soleus activity, closely followed by a bilateral burst of phasic tibialis anterior activity [33]. The combined effect of this muscle activity is to produce an external dorsiflexion moment at the ankles tending to rotate the body forwards over the feet. The hip abductors contribute to the control of frontal plane motion of the COM and the lateral loading/unloading mechanism during stance phase and gait. During gait initiation, anticipatory postural adjustments in the frontal plane shift the COP towards the swing foot, accelerating the COM towards the stance side (the last leg to leave the ground in gait initiation), allowing the swing foot to be lifted. Both the hip abductors and abductors contribute to this movement [21]. Gait initiation is divided into a preparatory and a stepping phase each of similar duration. The preparatory phase lasts from onset until the toe-off of the swing foot (SW TO) and is divided into two sub-phases, a release phase and an unloading phase. During the release phase the COP is moved postero-laterally towards the swing foot, increasing the horizontal GRF components that accelerate the COM in the opposite direction. This release phase lasts until the furthest point of posterolateral COP movement (maxCOP), when the COP abruptly changes direction, marking the start of the unloading phase. During the unloading phase, the COP is moved rapidly across to the stance foot, unloading the swing foot for toe-off. Swing leg toe-off marks the start of the stepping phase of gait initiation, which is sub-divided into single and double support phases (SSP and DSP). The SSP lasts from SW TO until initial contact of the swinging foot (SW IC), with DSP lasting from SW IC until toe-off of the original stance foot [28].

\subsection{Gaitrite 5.2.1 Introduction}

The GAITRite System is an electronic walkway utilized to measure the temporal (timing) and spatial (two dimension geometric position) parameters of its pressure activated sensors. The intent is utilizing the devices as measuring instruments for the events occurring during biped and quadruped locomotion [7]. Inferred parameters are easily obtained by applying common physics and math formulas to the directly measured temporal and spatial data i.e. calculate velocity, relationships between spatial and temporal events. As well as obtained in previous studies [8], the use of GAITRitec as a valid and reliable measurement of selected spatial and temporal parameters in adult gait is supported. It is proving to be an excellent tool to measure the functional characteristic of gait. Universities and medical centers all over the world are implementing this software into their operations. A new application, one that involves training and immediate feedback has recently been introduced, and it appears to have tremendous potential. The standard GAITRite software calculates numerous temporal and spatial parameters and displays them in tables and graphs. When synchronization to another device is desired, a 5 volt positive pulse is sent out of the carpet via the BNC cable at the instant the walkway becomes active [46]. This enables an EMG instrument and/or a 
Total hip replacement: structures modeling, gait analysis and report

European Journal Translational Myology - Basic Applied Myology 2012; 22 (1\&2): 69-121

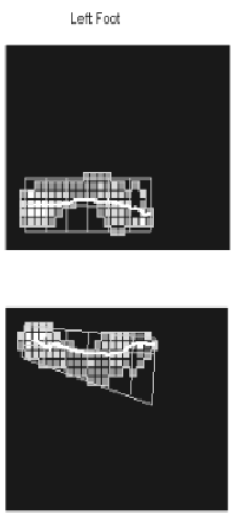

Right Foot

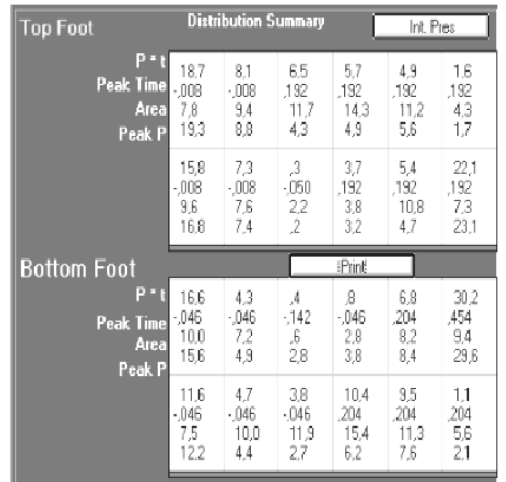

Fig 40 Pressure

video camera to turn on or to precisely record the socalled "time zero". While the "BNC out" option has been available for several years, and it has become invaluable to our clients, the ability to introduce a signal into GAITRite has been missing until today. The new "Analog in and integrated metronome (AIM)" option, enables signals to be introduced into the GAITRite walkway's firmware simultaneously with the footstep data. These accurate and objective events are combined with the temporal and spatial parameters numerically and graphically. When analyzed with the GAIT Raw software, individual sensor information relative to the aforementioned events can be visualized and exported. The GAIT Raw software module collects object positional data without regard to reciprocal gait or number of objects, thus any activity occurring on the carpet is captured. These data can be replayed in continuous mode or at various frame rates. The data can be exported either as: time, $\mathrm{x}$ and $\mathrm{y}$ coordinates,

\begin{tabular}{|c|c|c|c|c|c|c|c|}
\hline \multicolumn{4}{|c|}{ Step Time $[s]$ - operated } & \multicolumn{4}{|c|}{ Step Time [s] - healthy } \\
\hline lenght IC & $95 \%$ - & Mean & $95 \%+$ & lenght ic & $95 \%$ - & Mean & $95 \%+$ \\
\hline 0,054033 & 0,542983 & 0,57 & 0,597017 & 0,041854 & 0,501073 & 0,522 & 0,542927 \\
\hline \multicolumn{4}{|c|}{ Cycle Time $[s]$ - operated } & \multicolumn{4}{|c|}{ Cycle Time [s] - healthy } \\
\hline lenght IC & $95 \%$ - & Mean & $95 \%+$ & lenght IC & $95 \%$ - & Mean & $95 \%+$ \\
\hline 0,079374 & 1,050313 & 1,09 & 1,129687 & 0,090755 & 1,048623 & 1,094 & 1,139377 \\
\hline \multicolumn{4}{|c|}{ Step length $[\mathrm{cm}]$ - operated } & \multicolumn{4}{|c|}{ Step length $[\mathrm{cm}]$ - healthy } \\
\hline lenght IC & $95 \%$ - & Mean & $95 \%+$ & lenght IC & $95 \%$ - $-1-1-$ & Mean & $95 \%+$ \\
\hline 5,296983 & 51,71351 & 54,362 & 57,01049 & 3,865923 & 55,60704 & 57,54 & 59,47296 \\
\hline \multicolumn{4}{|c|}{ Stride length $[\mathrm{cm}]$ - operated } & \multicolumn{4}{|c|}{ Stride length $[\mathrm{cm}]$ - healthy } \\
\hline lenght IC & 95\% - & Mean & $95 \%+$ & lenght ic & $95 \%$ - $-1-1-10$ & Mean & $95 \%+$ \\
\hline 7,587743 & 108,3201 & 112,114 & 115,9079 & 9,407685 & 107,7382 & 112,442 & 117,1458 \\
\hline \multicolumn{4}{|c|}{ Velocity $[\mathrm{cm} / \mathrm{s}]$} & & & & \\
\hline lenght IC & 95\% - & Mean & $95 \%+$ & & & Age & Side \\
\hline 12,93743 & 97,11129 & 103,58 & 110,0487 & & & 57 & L \\
\hline
\end{tabular}

Fig 41 Single acquisition layout

AIM time and relative pressure value; or time, $\mathrm{x}$ and $\mathrm{y}$ coordinates, AIM time and on/off flag documenting the effectiveness of gait training has never been easier. At the beginning of the treatment regimen, GAITRite can identify the client's footfall patterns and gait parameters quickly and easily. Since cadence training has been proven to be an effective modality for kids and adults, the ability to set the metronome to a certain frequency, while monitoring the exact foot strike information is more important then ever. Immediate feedback is available for both the client and the clinician.

\subsubsection{Method}

The figure 41 gives an example of typical acquisition where are presented the patient walking on a carpet, engineers on the laptop controlling measurements and the physiotherapist, which is supervising movements of the subject. We get measurements in three

\begin{tabular}{|l|c|c|}
\hline PARAMETER DESCRIPTION & Total/Left & Right \\
\hline Step Count & 76 & \\
\hline Distance & 3761,4 & \\
\hline Ambulation Time & 46,16 & \\
\hline Velocity & 81,5 & \\
\hline Cadence & 98,8 & \\
\hline Step Time Differential & 0,02 & \\
\hline Step Length Differential & 2,757 & \\
\hline Cycle Time Differential & 0,001 & \\
\hline Step Time(sec) & 0,617 & 0,597 \\
\hline Step Length(cm) & 48,15 & 50,907 \\
\hline Cycle Time(sec) & 1,215 & 1,214 \\
\hline Stride Length(cm) & 99,401 & 99,966 \\
\hline HH Base Support(cm) & 14,431 & 14,453 \\
\hline Swing Time(sec) & 0,454 & 0,399 \\
\hline Stance Time(sec) & 0,761 & 0,815 \\
\hline Single Supp. Time(sec) & 0,399 & 0,454 \\
\hline Double Supp. Time(sec) & 0,361 & 0,366 \\
\hline Swing \% of Cycle & 37,4 & 32,9 \\
\hline Stance \% of Cycle & 62,6 & 67,1 \\
\hline Single Supp \% Cycle & 32,8 & 37,4 \\
\hline Double Supp \% Cycle & 29,7 & 30,1 \\
\hline Toe In / Out & $-8,6$ & -4 \\
\hline & & \\
\hline
\end{tabular}

\begin{tabular}{|l|c|c|}
\hline PARAMETER DESCRIPTION & Left & Right \\
\hline HeelOffOn Time & 0,057 & 0,065 \\
\hline HeelOffOn Perc & 4,7 & 5,4 \\
\hline Double Supp Load Time & 0,163 & 0,163 \\
\hline Double Supp Load \%GC & 16,5 & 13,4 \\
\hline Double Supp Unload Time & 0,16 & 0,203 \\
\hline Double Supp Unload \%GC & 13,2 & 16,7 \\
\hline Stride Velocity & 81,973 & 82,54 \\
\hline Step Len Std Dev & 3,038 & 2,948 \\
\hline Step Time Std Dev & 0,025 & 0,028 \\
\hline Stride Length Std Dev & 5,291 & 4,539 \\
\hline Stride Time Std Dev & 0,042 & 0,048 \\
\hline Swing Time Std Dev & 0,02 & 0,021 \\
\hline Stance Time Std Dev & 0,038 & 0,038 \\
\hline Stride Velocity Std Dev & 6,151 & 5,697 \\
\hline Single Supp Time Std Dev & 0,021 & 0,02 \\
\hline Double Supp Time Std Dev & 0,031 & 0,033 \\
\hline Heel Off On Std Dev & 1,124 & 1,322 \\
\hline Supp Base On Std Dev & 2,799 & 2,363 \\
\hline Foot Length & 22,7 & 23,6 \\
\hline Foot Width & 7,112 & 7,517 \\
\hline
\end{tabular}

Fig 42 Table of workbench parameters 
Total hip replacement: structures modeling, gait analysis and report

European Journal Translational Myology - Basic Applied Myology 2012; 22 (1\&2): 69-121
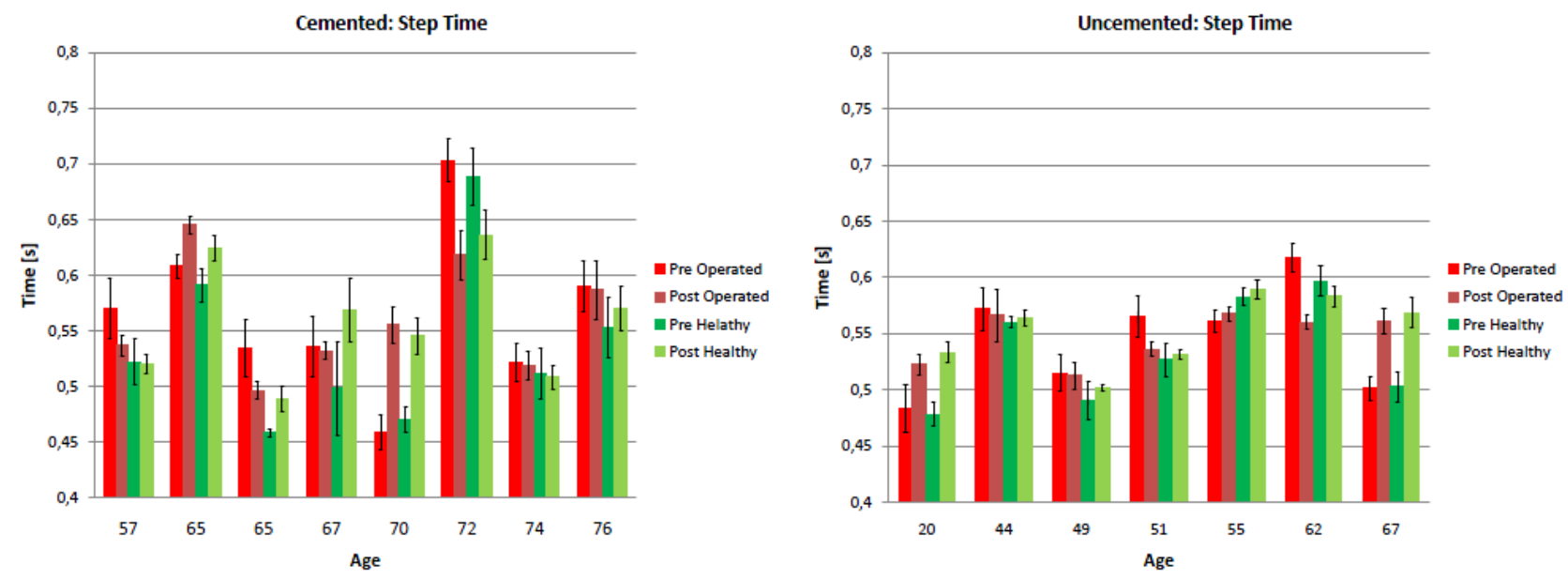

Fig 43 Temporal parameters comparisons
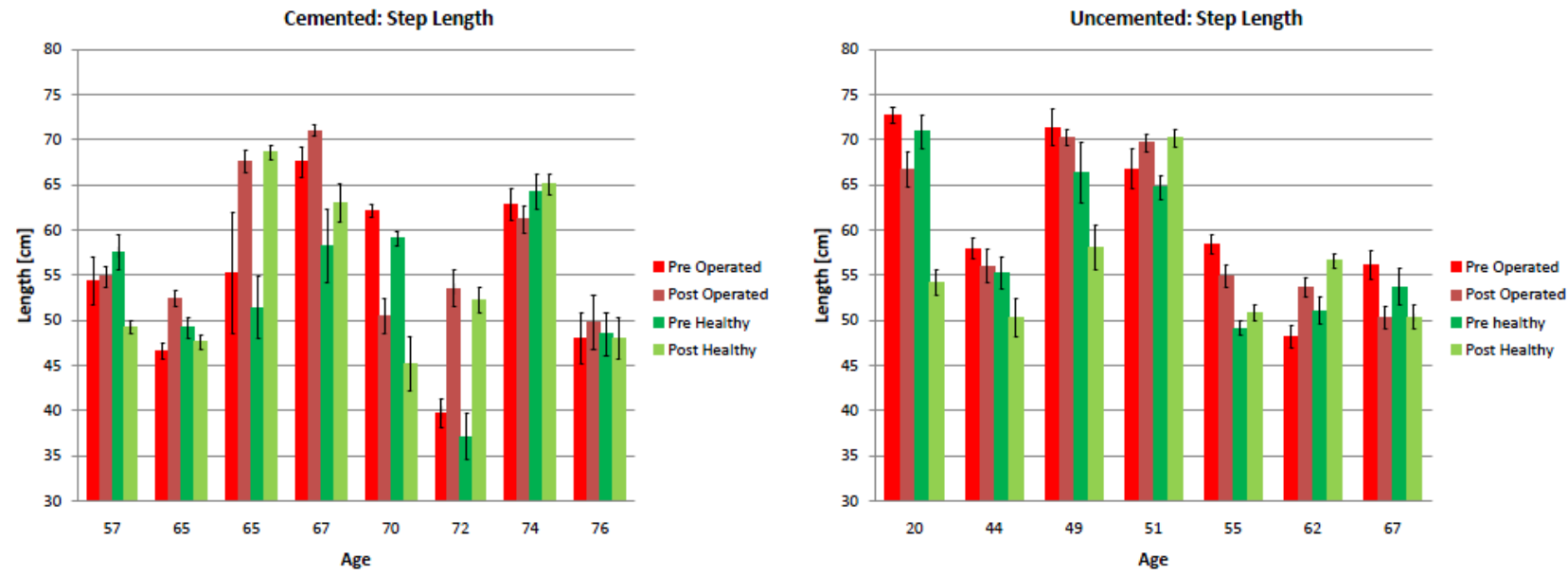

Fig 44 Spatial parameters comparisons

fundamental checkpoints: before the operation, after and one year later. The main work in these phases consists on collect information on the patients gait through the GAITRite system. According with the manual of the device, the subsequent method is adopted in order to store pre-elaborated data on a laptop and then process them in a suitable way for our purpose. All the parts of the system are assembled and the carpet is settled in the main room where the measurements are taken. References by black tape are fixed on the ground in order to go back to the length of the carpet. Once the patient is ready according to the physiotherapist, he starts walking on the sensor carpet meanwhile the data are stored in the computer system connected by usb cables. The patients walk begins at least 5 steps before the carpet to reproduce the normal stride behavior. Each one of the patients undergoing the gait analysis walks at least 6 times for both of the directions on the carpet. Thus, from a statistical point of view, it is supposed to be enough to get accurate information at the end of the analysis phase. The files in the computer are initially stored with .wlk extension, and grouped by project checkpoints. In a second while, the elaboration phase is transfered to department computers where these .wlk files are imported in the GAITRite software and managed. The result of each one of the walks is a layout of parameters as shown in Figure 42, a virtual path on the carpet and a pressure analysis (Figures 40 and 41). The overall prospect for the patient stride has been checked usually from physiotherapist to note for example asymmetries (stance percentage of gait cycle or increase in single support time that should be equal) Given that we handle 6 tries per patient, to associate single values the mean value for each one of the parameters has been calculated. Furthermore, the standard deviations have been obtained as well, useful for subsequent statistical considerations.

Among all the parameters available from GAITRite from which we got averages, a selection is just considered because of the significant use of these data comparing them with models and other observations. Therefore, the averages used as elaborated parameters are grouped by checkpoint, and subsequently stored in 
Total hip replacement: structures modeling, gait analysis and report

European Journal Translational Myology - Basic Applied Myology 2012; 22 (1\&2): 69-121

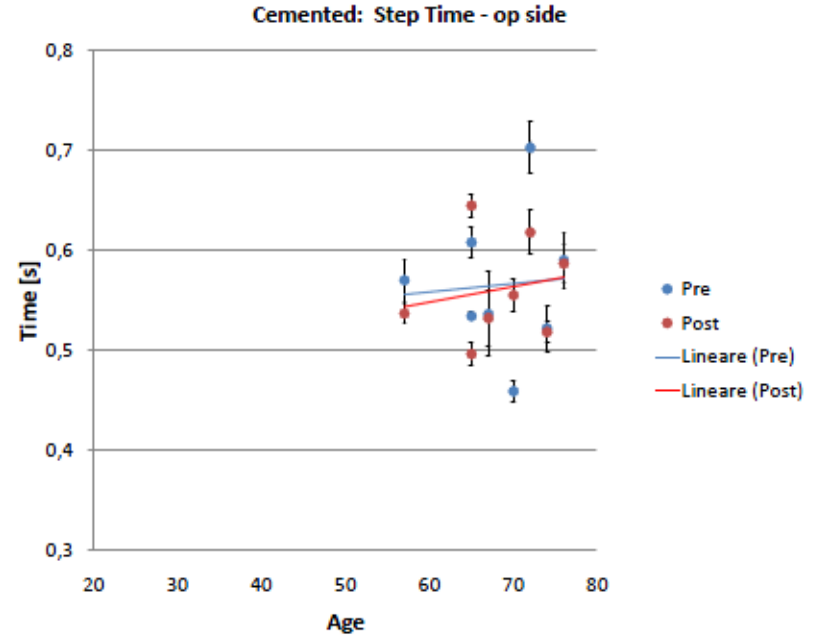

(a)

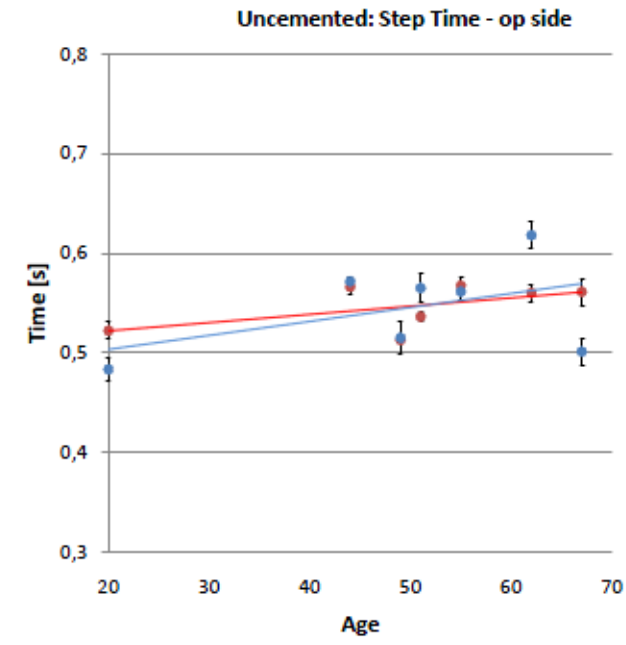

(b)

Fig 45 Scatter view for step time

the THR Project database. According to statistics principles, the parameters are represented in order to show for both of them estimated intervals on 95\% confidence (find out how in Appendix C). Thus mean values, lower and upper boundaries and IC lengths have been reported as typical layout for single measurement. Although data are few in quantity comparing with typical sample length, the method reported is adopted according many previous examples of study [39]. In the database of Microsoft Access 2007c the values have been collected in order to compare them among both the patients (classified according to the type of prosthesis used) and the different checkpoints for the single patient in the same chart to assess any differences due to the going by time. Besides the parameters already discussed, we get a virtual map of feet, quite similar to footprints, each one divided in 12 zones: one zone presents pressure values and a value of time for the standing time on the ground of the corresponding part of the foot. Furthermore, the twelve zones do not correspond same dimensions, thus the outcome for this measurements includes areas of reference as well. An average for the distinguished zone is also done, considering both the relative area of the footprint and the real value of pressure. Regarding the pressure and the path of the patient we do not store them in the database because it is possible to obtain a simple print out useful for the physicians or people involved in the project, especially from the rehabilitation point of view. Actually, taking a look of the print out, it is possible for the staff to notice weird aspects of the foot standing throughout all the standings phases and further for all tries that the patient accomplishes.

\subsubsection{Results}

Measurements are however grouped by type of implant and sorted by the period of acquisition. Data have been collected in Grensas hospital of rehabilitation and elaborated in department.

To point out any ties with anamnesis of patients, the groups have been ordered by age as well as the BMD layout presented. Day by day, the schedule for the project increases in number of patients, mainly helpful for this part of the work in which relevant importance lies on statistical significance. At the end of the next year, the observations from the last "measurement checkpoint" will be taken, stored and plotted as well together with the others.

In the single acquisition Figure 41 has been shown a classic layout for single measurement. It is possible to find out the values of interest and compare them with both patients' data from other acquisition periods and diverse patients result. Age and side of operation are however present. Charts have been done in order to underline potential trends in parameters and connections with the implant adopted. Only a selection of the available graphs is shown here (Figures 43 and 44), the entire layout has been reported in appendix to which please refer the reader. Checking step time charts of Figure 45 we notice an absence of significant trends concerning relations with implant utilized actually. This absence might be anyway expected due to wide number of factors setting in and makes gait data strictly connected to single patient conditions. Although there are no common paths, referring to temporal parameters, "step time" and "cycle time" for the uncemented group evidence in many subjects an achievement of more stable status, argued by closer values for healthy and operated side in the post op comparing with first acquisitions. The cemented group indeed reports minor differences in this sense, and in two cases (patients 72 and 74 years old) no significant changes have been noticed even though the operation has been done. Afterward, for spatial parameters we 
Total hip replacement: structures modeling, gait analysis and report

European Journal Translational Myology - Basic Applied Myology 2012; 22 (1\&2): 69-121

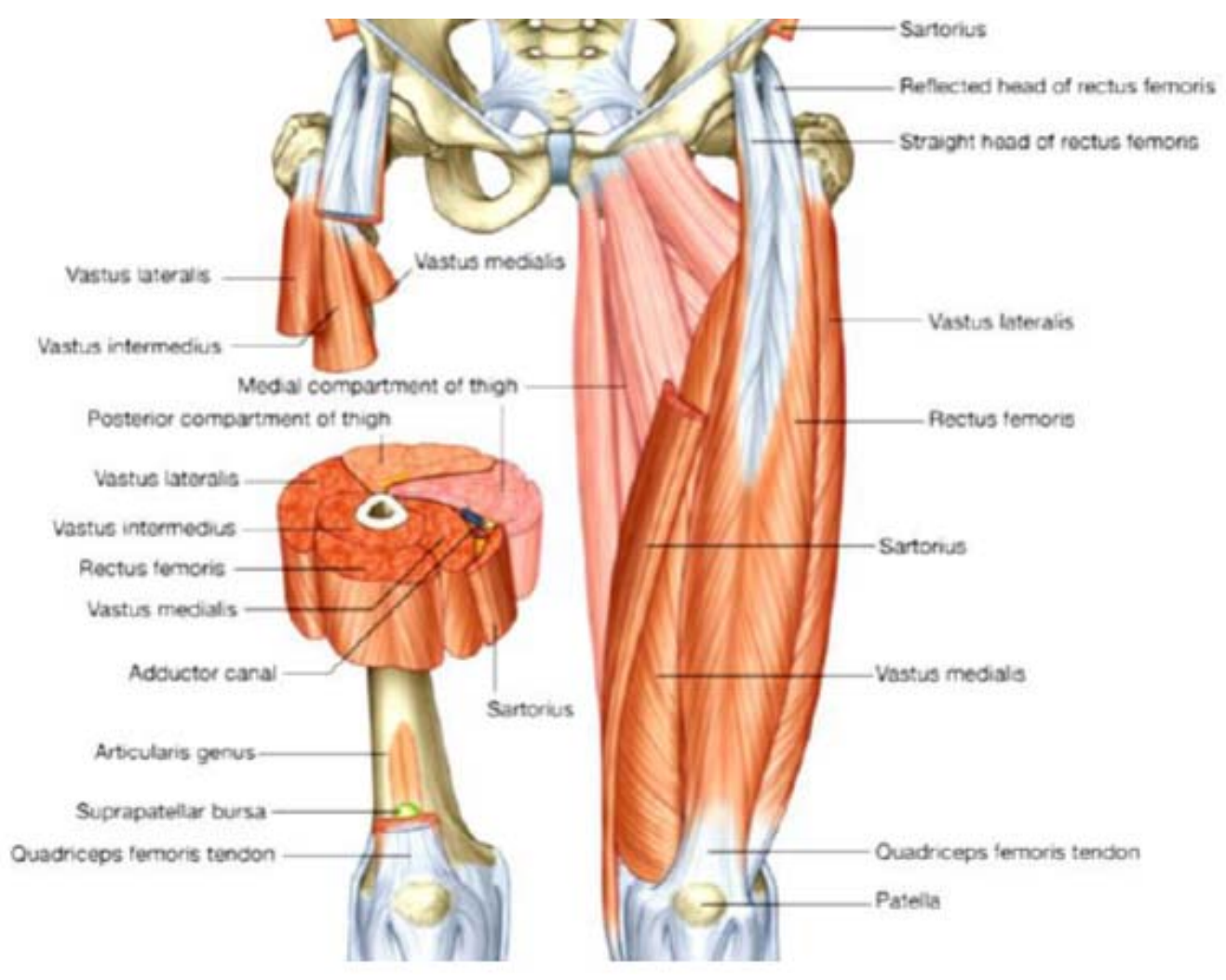

Fig 46 Anatomy of thigh

notice, at least in average, higher results for cementless group rather than cemented control list. As we said for previous parameters,

the evidence of more stability now is reached as well, and likely this kind of status can be due to benefited conditions for patients underwent THR in uncemented implant (please refer to charts 47). Therefore, not so serious conditions for the bone structure that indeed can bear the implant without medium, permits faster improvements in walk characteristics as well as noticed by physiotherapists during the routine follow up in rehabilitation. A different view on the overall prospect has been given by following graphs $45 \mathrm{a}$ and $45 \mathrm{~b}$. Only values of step time for the operated side are considered. The purpose of this visualization is to point out a major dispersion that lies in the cemented group of patients regarding the side treated with THR. Despite the age, which is normally distributed in a lower range for cementless list, especially for the time in consideration there is more variance of results in the left one (cemented group). Furthermore, IC95\%, represented by black lines and boundaries, are much larger: a possible meaning lies in scarce repeatability of measurements for each patient among the roughly six tries in an acquisition period (IC95\% reflects the variability of the patients in his own tries) in cemented case. Linear trends are though displayed to summarize where pre op values are lower than post op or vice versa, meant by red and blue lines in respective positions: e.g. red line beneath blue one means post op reflects a lower value. After what has been argued a final discussion can be held. The gait rite data elaborated are partial as the project will go ahead with next year measurements. Although the results are not complete yet, the given prospect (deepened in quantity in appendix) claims the possibility to be a useful tool for the rehabilitation due to observations on single patients' changes in parameters of the walk. Conversely, it appears improbable to link this kind of measurement to implants used possibly to foresee the suited implant respecting patient's conditions. These, actually, seem too different as trends are not individuated yet. Apart from given interpretation about variability, aging and higher values, thus we realize that these measurements should be properly considered in assessing patient improvements during the rehabilitation.

\section{3 sEMG and modeling correlation}

This section of the chapter encloses two different aspects: modeling tools use and sEMG data obtained by KINE Pro system. In order to correlate interested muscles morphology and muscle functionality in patients undergoing THR, we take advantage from both the elaborations: the morphology is figured out from MIMICS modeling tools, the functionality through sEMG and gait analysis, furthermore we consider as checkpoints "preop" and "6 weeks later" respecting which information are grouped by. 
Total hip replacement: structures modeling, gait analysis and report

European Journal Translational Myology - Basic Applied Myology 2012; 22 (1\&2): 69-121

\subsection{1 sEMG briefing}

Electromyography (EMG) is a technique for evaluating and recording the electrical activity produced by skeletal muscles. It is about detecting the electrical potential generated by muscle cells when these cells are electrically or neurologically activated. The signals can be analyzed to detect medical abnormalities, activation level, and recruitment order or to analyze the biomechanics of human or animal movement. There are two kinds of EMG in widespread use: the surface EMG and intramuscular (needle and fine-wire) EMG. The second may be considered too invasive or unnecessary in many cases. Instead, a surface electrode may be used to monitor the general picture of muscle activation, as opposed to the activity of only a few fibers as observed using an intramuscular are EMG. The surface technique is used in a number of settings; for example, in physiotherapy clinics where muscle activation is monitored and patients have an auditory or visual stimulus to help them know when they are activating the muscle (biofeedback). There are many applications for the use of EMG. It is used clinically for the Diagnosis of Neurological and Neuromuscular Problems (even in Dentistry); Diagnostically by Gait Laboratories and by Clinicians trained in the use of Biofeedback or Ergonomic Assessment; in many types of Research Laboratories, including those involved in Biomechanics, Motor Control, Neuromuscular

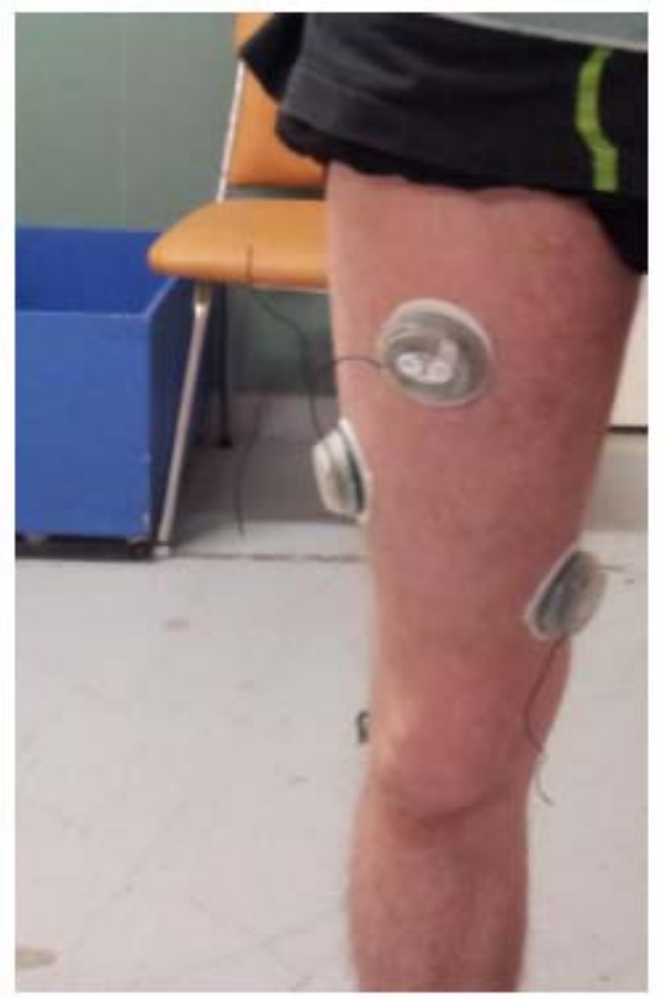

Fig 47:Sensors disposition
Physiology, Movement Disorders, Postural Control, Sports, Training and Physical Therapy. One of the first examples to offer commercially a Totally Wireless SEMG Solution is the adopted system, where the interactions between the sensor-unit and the receiving unit are completely wireless. In this way it is possible to bypass many of the limitations, wires and cables impose on measuring subjects under "normal conditions", i.e. at work, in sports, resting or furthermore during rehabilitation.

\subsubsection{Setup on muscles}

Small electrical currents are generated by muscle fibres prior to the production of muscle force. These currents are produced by the exchange of ions across muscle fiber membranes, a part of the signaling process for the muscle fibers to contract. The signal called the electromyogram (EMG) can be measured by applying conductive elements or electrodes to the skin surface, or invasively within the muscle. Surface EMG is the more common method of measurement, since it is noninvasive and can be conducted by personnel other than Medical Doctors, with minimal risk to the subject. Measurement of surface EMG is dependent on a number of factors and the amplitude of the surface EMG signal (sEMG) varies from the microV to the low $\mathrm{mV}$ range [4]. The amplitude and time and frequency domain properties of the sEMG signal are dependent on factors such as:

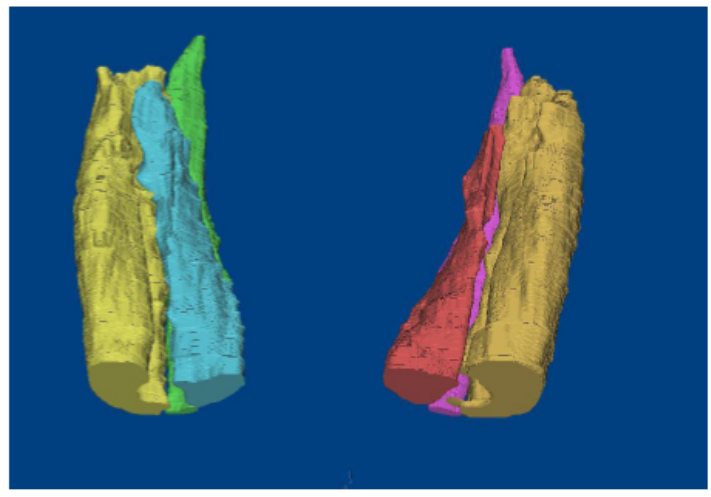

Fig 48 Modeling of muscles

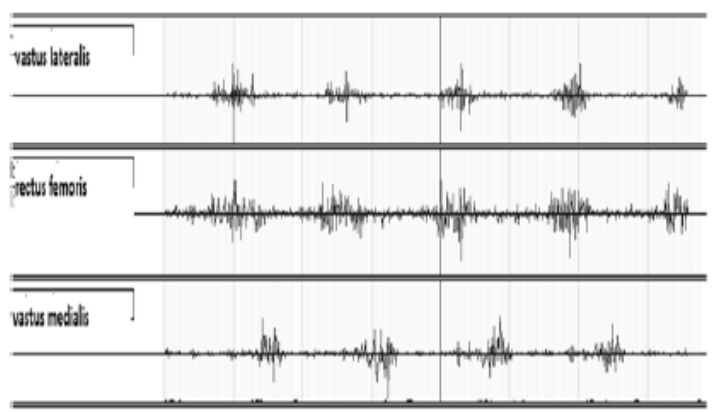

Fig 49 Muscle EMG from Kinepro of muscles 
- Timing and intensity of muscle contraction.

- Distance of the electrode from the active muscle area.

- Properties of the overlying tissue (e.g. thickness of overlying skin and adipose tissue).

- Electrode and amplifier properties.

- Quality of contact between the electrode and the skin. In most cases, information on the time and intensity of muscle contraction is desired. The remainder of the factors only exacerbates the variability in the EMG records, making interpretation of results more difficult. Nevertheless, there are methods to reduce the impact that non- muscular factors have on the properties of the EMG signal. For example, much of this variability in the sEMG signal can be minimized through:

- Using the same electrodes and amplifier (i.e. same signal conditioning parameters).

- Ensuring consistency in the quality of contact between the electrodes and the skin.

Within subjects, the variability of the sEMG signal can also be reduced in consecutive recording sessions by placing the electrodes over the same skin location. In addition, there are other methods of normalizing the EMG signal to reduce the variability both within and between subjects. Many of the most important issues relating to the acquisition and analysis of the sEMG signal were recently addressed in a multi-national consensus initiative called SENIAM: Surface EMG for the Non-Invasive Assessment of Muscles [20]. Measuring and accurately representing the sEMG signal depends on the properties of the electrodes and their interaction with the skin, amplifier design, and the conversion and subsequent storage of the EMG signal from analog to digital form (i.e. A/D conversion). The quality of the measured EMG is often described by the ratio between the measured EMG signal and unwanted noise contributions from the environment. The goal is to maximize the amplitude of the signal while minimizing the noise. Assuming that the amplifier design and process of $\mathrm{A} / \mathrm{D}$ conversion exceed acceptable standards, the signal to noise ratio is determined almost exclusively by the 4 electrodes, and more specifically, the properties of the electrodeelectrolyte-skin contact.

\subsubsection{Method}

The physiotherapist sets electrodes on the patient body, in particular on a muscle group that occupies the front and sides of the thigh and is primary extensor of the knee and hip flexion implied in, noticeable in Figure 48. It is composed of four parts: rectus femoris, vastus lateralis, vastus medialis, and vastus intermedius.

These parts connect the ilium and femur to a common patellar tendon, which passes over the front of the knee and attaches to the patella (knee cap). Three muscles are studied: rectus femoris, vastus lateralis and vastus medialis according with the SENIAM project (Surface ElectroMyoGraphy for the Non-Invasive Assessment of Muscles): rectus femoris: Origins straight head from anterior inferior iliac spine. Reflected head from groove above rim of acetabulum. It has the insertion on the proximal border of the patella and through patellar ligament. It supports the extension of the knee joint and flexion of the hip joint. (Figure 46) The electrodes need to be placed at $50 \%$ on the line from the anterior spina iliaca superior to the superior part of the patella in the direction of the line from the anterior spina iliaca superior to the superior part of the patella. The fixation on the skin is in (Double sided) tape, rings or elastic bands. The Rectus femoris muscle is one of the four quadriceps muscles that sit in the middle of the front of the thigh. It is responsible for knee extension and hip flexion. Vastus lateralis: Proximal parts of intertrochanteric line, anterior and inferior borders of greater trochanter, lateral lip of gluteal tuberosity, proximal half of lateral lip of linea aspera, and lateral intermuscular septum. Insertion in proximal border of the patella and through patellar ligament. Function of extension of the knee joint. Electrodes need to be placed at $2 / 3$ on the line from the anterior spina iliaca superior to the lateral side of the patella, oriented in the direction of the muscle fibers. vastus medialis: Distal half of the intertrochanteric line, medial lip of line aspera, proximal part of medial supracondylar line, tendons of adductor longus and adductor magnus and medial intermuscular septum. Insertion in proxypatellar ligament. Stands the extension of the knee joint. Electrodes need to be placed at $80 \%$ on the line between the anterior spina iliaca superior and the joint space in front of the anterior border of the medial ligament. The orientation is almost perpendicular to the line between the anterior spina iliaca superior and the joint space in front of the anterior border of the medial ligament (Figure 47). Spiral CT-scans used in modeling work before and after operations are always the base of modeling tools. The slices from the CTscan are uploaded into the MIMICS software were thresholding and segmentation were done. Thresholding was used to create a gap across bonesmuscles edges trough HU scale separation obviously given that HUs describe the density of structures, where bone and muscles appear sensibly different. Segmentation tools were used to distinguish each muscle in both legs. To disjoin the muscles, it was important to trace them by mimics tools. This arduous tracing has been succeeded by careful observation on the CT-slices comparing apparent muscles shape with anatomy information of common knowledge (Figure 48).

Therefore the edges of each muscle have been figured out, evidencing the shape for both the muscles in consideration. The last part already described beneath all the comparisons among muscles and between scans of different period of acquisition. By that, 3D models have been realized and density of the muscles found out. The density is actually an index of the health 
Total hip replacement: structures modeling, gait analysis and report

European Journal Translational Myology - Basic Applied Myology 2012; 22 (1\&2): 69-121

\begin{tabular}{|c|c|c|c|c|}
\hline \multirow[t]{2}{*}{ DENSITY [HU] P1 } & \multicolumn{2}{|c|}{ Before operation } & \multicolumn{2}{|c|}{ After operation } \\
\hline & Healthy & Operated & Healthy & Operated \\
\hline Rectus femoris & 49,96 & 43,05 & 44,55 & 42,38 \\
\hline Vastus medialis & 44,75 & 43,22 & & \\
\hline Vastus lateralis & 46,49 & 45,14 & & \\
\hline DENSITY [HU] P2 & Before & eration & After op & beration \\
\hline & Healthy & Operated & Healthy & Operated \\
\hline Rectus femoris & 55,57 & 49,68 & 54,62 & 50,88 \\
\hline Vastus medialis & 52,26 & 50,29 & & \\
\hline Vastus lateralis & 51,36 & 50,48 & & \\
\hline
\end{tabular}

Table 6 Densities of muscles

status of the muscle, because of the connection between functional efficiency and mass yielded. The density has been calculated as outcome of the modeling protocol for preoperative and postoperative CT scans for the muscles, currently only for rectus femoris both of them. Kine pro subsequently was used in the second period of acquisition, with equal setting on the patients, especially for sensors positions. The Kine Pro software associates to the walk tracks of EMG for each one of the muscles distinguishing healthy and operated side. However, the traces are grouped by patient, side and muscle given always that for period of acquisition all data are sorted by.

The software calculates values of interest for the EMG path as average, standard deviation, minimum and maximum values in microvolt (Figure 49).

\subsubsection{Results}

This section reports the outcome of a work that contains modeling and EMG analysis prerogatives. Thus likely it would be such an important development for the project in general once patients will reach the one year period of measurement. The results are indeed partial observations and collections on the verge of being compared to the new acquisitions after proper period of rehabilitation. As depicted subsequently, elaborations concern an example given of two patients both right sides operated which have been analyzed trough CT scan preoperative and postoperative, sEMG preoperative and six weeks later.

The layout of the work concerns usually tables reporting density of muscles and EMG traces, with resume values and possibly particular parameters. The Table 6 lists densities values for both the patients and would be analyzed in correspondence of new data since one year CT scan will be available. Muscles are normally displayed with $\mathrm{HU}$ values between 50 and $100 \mathrm{HU}$ though within a normal muscle belly there are also other tissue elements such as connective and fat which are coded with much lower $\mathrm{HU}$ values. This fact explains the wide range of values present inside a data set and suggests the definition of various intervals to study muscle structural changes [24]. The values we got in measurements cross down or roughly lie on the limit of denervated muscles, for the first, obviously underlined in operated side thighs, meaning of major use of the other thigh on which subjects incorrectly weigh. Referring in particular to rectus femoris values, for operated side both the patients and healthy for the first one actually denote low dense/atrophic muscle fibers comparing with previous study on denervated muscles HU in literature [37]. Probably future
Patient 1 sEMG LAYOUT
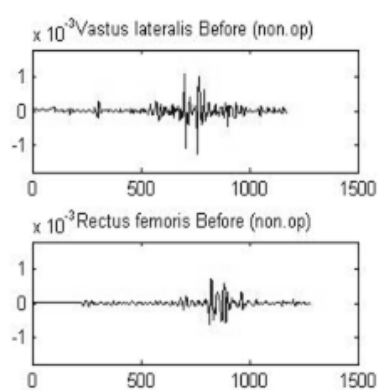

$\times 10^{-3}$ Vastus medialis Before (non.op)

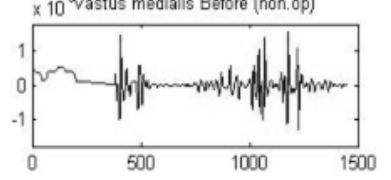

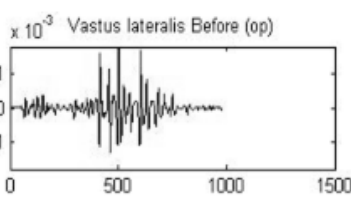

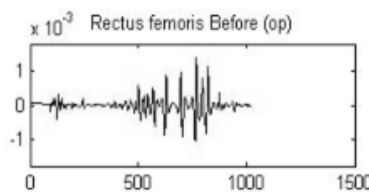

$\times 10^{-3}$ Vastus medialis Before (op)

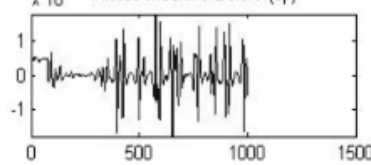

(a)
Patient 1 sEMG LAYOUT postoperative
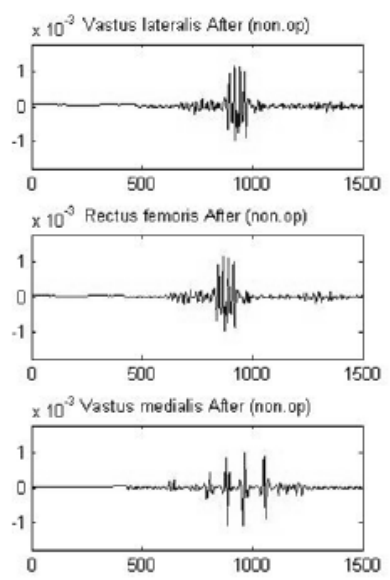

(b)
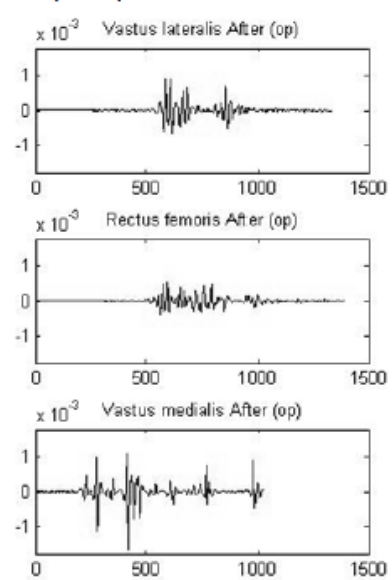

Fig 50 Patient 1 sEMG 
Total hip replacement: structures modeling, gait analysis and report

European Journal Translational Myology - Basic Applied Myology 2012; 22 (1\&2): 69-121

measurements will denounce a sort of recovery for muscle mass, growth of muscle and connective tissue as well due to normal distribution of load stood by the fibers, thus a consequence on both legs of normal and efficient walk stride can be expected, improved by rehabilitation and time went by the operation. sEMG traces are here represented, in Figures 50 and 51, and however distinct in checkpoints and healthy and operated side. The software provides tools of signal elaboration as users might seek particular meaningful parameters. Analyzing the electric activity for the first patient in example, before the operation denounces an imbalance in activity more prominent on the operated side. Even though postoperative patterns are sensibly lower in average, a recovered balance between the two legs seems has been found, indication of normal establishment back on the way. The second patient preoperative EMG path indicates overusing of rectus femoris and vastus medial on the operated side due likely to dysfunction of gluteus muscles after period along the patient in superior area of hip painful effort had made contributing to upper muscles denervation. Instead, successful operation and patient recovery have been proven by achievement of balance in electric activity although paths are still different in form. To get a wide realization of goal section, which actually would authorize staff making joint observations from electrical and morphological acquisitions, we will wait new CT scan and Kine pro acquisition in one year available. Despite absence of future measurements, partial work conclusions let us foresee an important way of rehabilitation assessment concentrated on electrical acquisition and modeling tool in order to associate morphological and functional information getting a complete layout on the thigh functionality, given that this section provides anyway most of information comparable with gait analysis obtainment.

\section{STORE AND REPORT}

\subsection{Introduction}

Patients undergoing the Total hip replacement, participating to the project, are increased in quantity day by day. Huge series of data has to be stored and reported in order to avoid a waste of functionality and clarity of measurements and elaborations. Therefore, as the project is going ahead, the main necessity is came a functional storing and report. It is common knowledge that the visual interface through which usually results are shown is almost more important than the own hidden work. Thus the opportunity to find a manner to communicate with people involved in the project has to be considered. The database moreover provides a quick way to control all the data, of course due to the storing manner, and always the possibility to print needed information out. Measurements and elaboration results are stored on server drivers provided by the hospital and managed according to different privileges of users' access. Each patient represents one subfolder of the main project path. In those, files derived by different branches of work are distinguished and stored respectively by source of study and sorted by period of acquisition and elaboration (preop, 6 weeks later and one year later). To get an overall prospect on the project a database was created on Microsoft Access 2007 environment, a DBMS element (stands for "Database Management System") In short, a DBMS is a database program. Technically speaking, it is a software system that uses a standard method of cataloging, retrieving, and running queries on data. The DBMS manages incoming data, organizes it, and provides ways for the data to be modified or extracted by users or other programs. Some DBMS examples include MySQL, PostgreSQL, Microsoft Access, SQL Server, FileMaker, Oracle, RDBMS, dBASE, Clipper, and FoxPro. Since there are so many database
Patient 2 sEMG LAYOUT preoperative

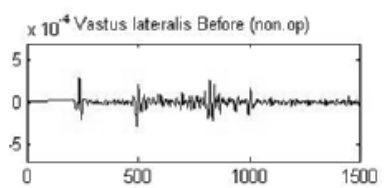

$\times 10^{-4}$ Rectus femoris Before (non.op)

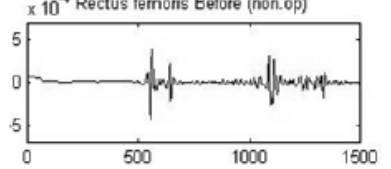

$\times 10^{-4}$ Vastus medialis Before (non.op)

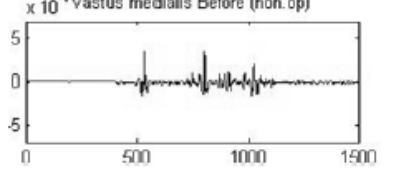

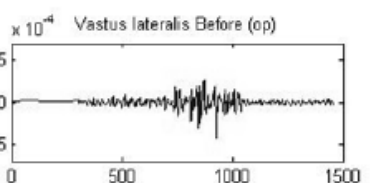
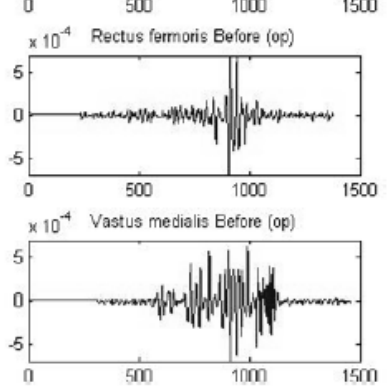

(a)
Patient 2 sEMG LAYOUT postoperative
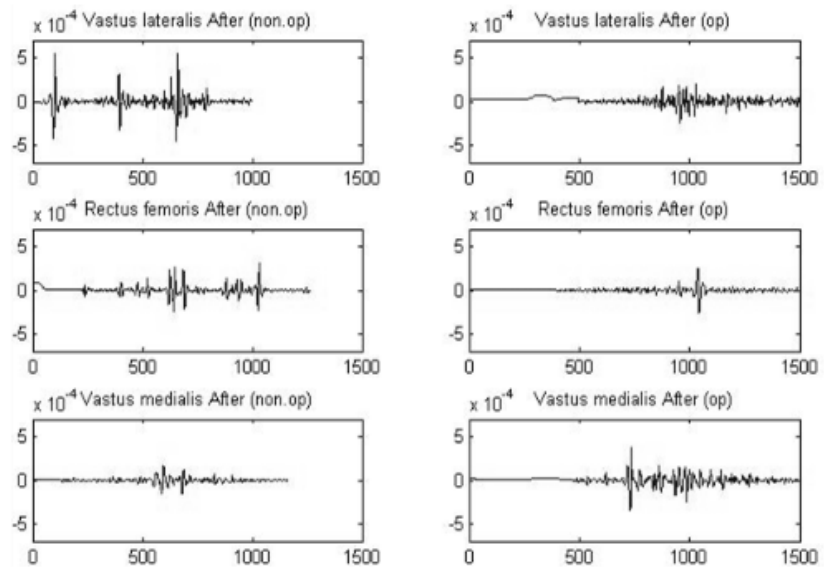

(b)

Fig 51 Patient 2 sEMG 
management systems available, it is important for there to be a way for them to communicate with each other. For this reason, most database software comes with an Open Database Connectivity (ODBC) driver that allows the database to integrate with other databases. The primary goal of such a system is to provide an environment that is both convenient and efficient to use in retrieving and storing information. A database management system (DBMS) is designed to manage a large body of information. Data management involves both defining structures for storing information and providing mechanisms for manipulating the information. In addition, the database system must provide for the safety of the stored information, despite system crashes or attempts at unauthorized access. If data are to be shared among several users, the system must avoid possible anomalous results due to multiple users concurrently accessing the same. data. A major purpose of a database system is to provide users with an abstract view of the data. That is, the system hides certain details of how the data are stored and maintained. Thereby, data can be stored in complex data structures that permit efficient retrieval, yet users see a simplified and easy-to-use view of the data. The lowest level of abstraction, the physical level, describes how the data are actually stored and details the data structures. The next-higher level of abstraction, the logical level, describes what data are stored, and what relationships exist among those data. The highest level of abstraction, the view level, describes parts of the database that are relevant to each user; application programs used to enter database form part of the view level.

\subsection{DBMS lexis}

The overall structure of the database is called the database schema. The schema specifies data, data relationships, data semantics, and consistency constraints on the data. Underlying the structure of a database is the logical data model: a collection of conceptual tools for describing the schema. The entityrelationship data model is based on a collection of basic objects, called entities, and of relationships among these objects. The set of all entities of the same type and the set of all relationships of the same type are termed an entity set and a relationship set, respectively. Like the entity-relationship model, the object-oriented model is based on a collection of objects. An object contains values stored in instance variables within the object. An object also contains bodies of code that operate on the object. These bodies of code are called methods. The only way in which one object can catch the data of another object is by invoking a method of that other object. This action is called sending a message to the object. Thus, the call interface of the methods of an object defines that object's externally visible part. The internal part of the object, the instance variables and method code, are not visible externally. The result is two levels of data abstraction, which are important to abstract away (hide) internal details of objects. Object-oriented, data models also provide object references which can be used to identify (refer to) objects. In record based models, the database is structured in fixed-format records of several types. Each record has a fixed set of fields. The three most widely accepted record-based data models are the relational, network, and hierarchical models. The relational model is very widely used. Databases based on the relational model are called relational databases. It uses a collection of tables (called relations) to represent both data and the relationships among those data. Each table has multiple columns, and each column has a unique name. Each row of the table is called a tuple, and each column represents the value of an attribute of the tuple. The size of a database can vary widely, from a few megabytes for personal databases, to gigabytes (a gigabyte is 1000 megabytes) or even terabytes (a terabyte is 1000 gigabytes) for large corporate databases. The information in a database is stored on a non-volatile medium that can accommodate large amounts of data; the most commonly used such media are magnetic disks. Magnetic disks can store significantly larger amounts of data than main memory, at much lower costs per unit of data. To improve reliability in mission-critical systems, disks can be organized into structures generically called redundant arrays of independent disks (RAID). In a RAID system, data are organized with some amount of redundancy (such as replication) across several disks. Even if one of the disks in the RAID system were to be damaged and lose data, the lost data can be reconstructed from the other disks in the RAID system. Logically, data in a relational database are organized as a set of relations, each relation consisting of a set of records. This is the view given to database users. The underlying implementation on disk (hidden from the user) consists of a set of files. Each file consists of a set of fixed-size pieces of disk storage, called blocks. Records of a relation are stored within blocks. Each relation is associated with one or more files. Generally a file contains records from only one relation, but organizations where a file contains records from more than one relation are also used for performance reasons. One way to retrieve a desired record in a relational database is to perform a scan on the corresponding relation; a scan fetches all the records from the relation, one at a time. Accessing desired records from a large relation using a scan on the relation can be very expensive. Indexes are data structures that permit more efficient access of records. An index is built on one or more attributes of a relation; such attributes constitute the search key. Given a value for each of the search-key attributes, the index structure can be used to retrieve records with the specified search-key values quickly. Indexes may also support other operations, such as fetching all records 
whose search-key values fall in a specified range of values. A database schema is specified by a set of definitions expressed by a data definition language. The result of execution of data-definition language statements is a set of information stored in a special file called a data dictionary. The data dictionary contains metadata, that is, data about data. This file is consulted before actual data are read or modified in the database system. The data-definition language is also used to specify storage structures and access methods. Data manipulation is the retrieval, insertion, deletion, and modification of information stored in the database. A data-manipulation language enables users to access or manipulate data as organized by the appropriate data model. There are basically two types of datamanipulation languages: Procedural data-manipulation languages require a user to specify what data are needed and how to get those data; nonprocedural data manipulation languages require a user to specify what data are needed without specifying how to get those data. A query is a statement requesting the retrieval of information. The portion of a data-manipulation language that involves information retrieval is called a query language. Although technically incorrect, it is common practice to use the terms query language and data-manipulation language synonymously. Database languages support both data-definition and datamanipulation functions. Traditionally database systems have been designed to support commercial data, consisting mainly of structured alphanumeric data. In recent years, database systems have added support for a number of nontraditional data types such as text documents, images, and maps and other spatial data. The goal is to make databases universal servers, which can store all types of data. Rather than add support for all such data types into the core database, vendors offer add-on packages that integrate with the database to provide such functionality.

\subsection{Structure and screenshots}

The database has been developed in Access 2007, work environment of Microsoft. Structure and layout of the database respect the work organization, as well as the work in project is divided in. As clarification, we better consider a diagram whose each part represents a development environment for elaborations and reports. Mainly, most of the objects attached are included in the database, so they actually represent program belongings. Otherwise, files that change day by day are reported through their path in the hospital server drive in order to mirror any upgrading, directly done on the files. Privileges to access the database (hence DB stands for database) are managed by the information technology department of the Hospital. This control indeed assigns to each user different available actions: user of biomedical engineering department are able to operate in write-read mode. Users from other departments of the hospital, besides seldom exceptions, are allowed to use the DB in read modality or to type data of new patients concerning their anamnesis. The main menu is divided briefly in two parts and represents a suited workbench for users. On the left of the main form indeed, interactive buttons lead to working sections of the program, otherwise the right part is characterized by interactive buttons for service actions forms opening, where actually files have been stored aiming to the path source in the hospital server. The top button, however on this right side of the screen, provides a program user manual. Subsequently, clicking the button beneath the manual one, it is possible to accede in a working environment like library as users had needs to keep easy access for certain files, which are common use for all of them. In poor words, it is supposed to report important files relative to staff involved, the work flow of surgeons in operation rooms and other general details. Further by clicking the below button, which lies on the left bottom of them form, are shown protocols inherent methods adopted for the project purpose. On the bottom left hyperlinks connect the project DB to websites of Reykjavik University of Iceland and Landspitali national hospital. Furthermore on the left part, clicking form buttons it is possible to implement preliminary and main actions performed by users. Thus, the top button "New patient" is obviously used to type information of new patients as Kennitala, patient name, weight, height and specially kind of implant and operated side. It has been foreseen an inclusion of files from surgeons in case helpful for them, once again aimed by path source in hospital server. The subsequent section has been created in order to change the previous information due to errors as well. The button below, "Start work" leads users to the patient workbench, passing throughout patients name consulting, otherwise check lists of patients grouped by kind of implant have been yielded by the programmer, reflecting the intent to compare patients undergone THR mainly distinguished by implant. Hence, selecting the desired patient, users accede to the overall prospect page depicted in Figure 52, actually the only one form that report every part of the project and behaves as consulting and storing library for the engineers. On the layout we can always figure out

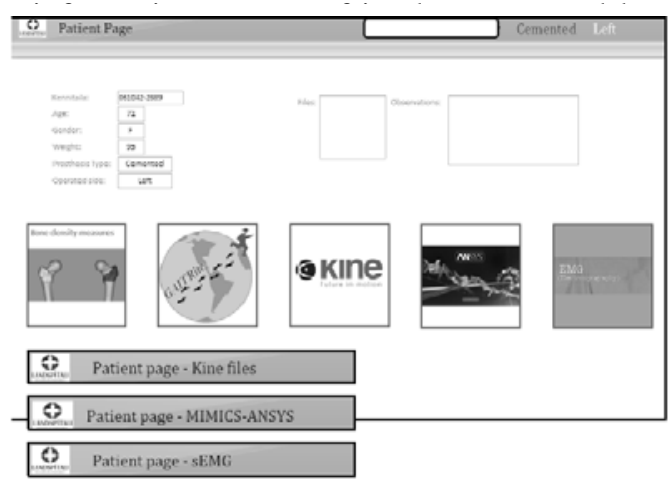

Fig 52 Patient page 


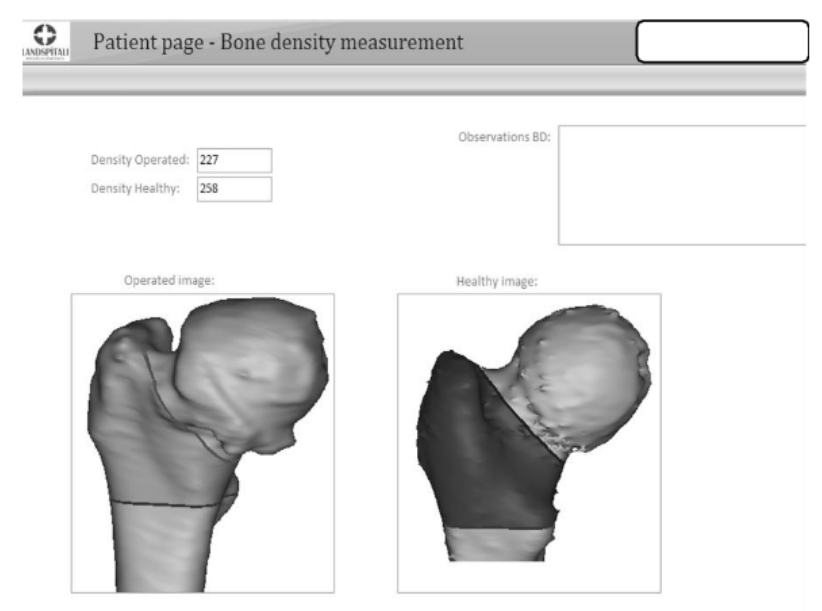

Fig 53 BMD form.

order to be always available even for a future print. Parameters in consideration are BMD healthy and BMD operated in HU, got through Mimics mask two dimensions analysis that can be transcribed in suited number cells, which are programmed to round entire numbers. "Observations BD" is a text box useful to explain, in case of necessity, particular considerations on the BMD output. Notice that on the top right of the view, as already seen for the joint page, there are always reported patient name, implant and operated side. At the bottom, the print button can be clicked to obtain a paper layout specific for this section. The paper summary contains all information of the patient, included in "Start work" section, and in addiction data and images of BMD forms, hospital logo, university brand and date of printing (Figure 53).

Gait rite: The statistic process of elaboration for data, derived from proper Gait rite software, has given principally as outcomes averages for each acquisition period and intervals of confidence per measurements. A deepened discussion about the parameters was already done in the fifth chapter, to which return for consultation. However, data insertion has been structured in group for period of acquisition, and distinguished by color, to indicate healthy and operated leg respectively blue and red shades. Therefore, once users have accomplished the typing phase, at the form bottom are charts filled by relative values inserted. Intervals of confidence are on the right, contained in a proper excel file whom is linked to the database (the statistical process can change day by day, thus updates are mirrored in the page). KineMimics and Ansys-sEMG: Usually DBMS are functional to store and report every kind of information. In this case, elaborations have been accomplished in proper softwares or excel sheets where it is supposed to handle data in an easier way and manage them later through the DB. Furthermore, each one of the pages can be saved as a pdf file containing all suited information, in any print out case. Obviously changes, modifies or anything else have been be done both in the source file and trough the stored object of the program, be- cause of the link nature of objects inserted in the DB.. Resume: The resume form, which is supposed to be always available for users that want to glance results, actually depicts the whole prospect concerning gait rite data and BMD sections. The form collects charts already reported in results chapters, where different necessities have been mirrored. BMD charts lie on the left side of the form, where bone density for cemented and uncemented implants has been shown. Averages in HU are below, with two charts and the correspondent legend indicating red and yellow shapes of columns for operated and healthy side respectively. In order to evince potential trends correlated to Age, these graphs and gait rite charts as well have been structured as agerelation reporting on the $\mathrm{x}$-axis. On the right side, the page program contains as anticipated gait rite outcome in charts related with patient age. Scatter points represent a single measure, and obviously will collect values of the next year as well. A stamp for the whole prospect can be realized pushing the suited bottom leading to print view and printer properties.

\section{CONCLUSIONS AND PERSPECTIVES}

The thesis has been based on the author work during abroad permanence in Iceland, derived by the THR project sight in. This study program represents a continuous application in progress for the national hospital of Iceland, in collaboration with Reykjavik University Biomedical Engineering Department. The drawn document actually acts as main and first outcome of the project, since six months after the beginning the thesis has been written. The work accomplished comprehends many aspects of biomedical engineering issues: a research of certain metrics tools in order to support hospital board for choice of implants adopted, nevertheless people involved in rehabilitation, incorporates biomaterial sector, image processing, elaboration data, and reporting system nowadays of main importance among world of health assistance providers. In synthesis the aims for issues of the project are: Metrics of support for surgeons to define a standard protocol for decisions of implants to apply. BMD evaluation might surely represent one auxiliary objective aid to the normal way of proceeding in prosthesis choice. Fracture risk and strain test elaboration will coach surgeons in operations avoiding intra operative failure. metrics of support for people involved in rehabilitations. Gathered data represent a scientific overview on patients' conditions pre and post operation, a manner to assess any improvements during physiotherapist follow up. Conditional correlations with chosen implants would be underlined. Correlations between muscles status and gait advanced observations by 
sEMG, abnormally accomplished due to pathologic walk changes in inefficient stride. The research aims to figure out the connection of prior and posterior operation muscles characteristics and sEMG traces meanwhile rehabilitation work. Creation and development of graphic and functional interfaces, according to staff necessities, in order to functionally store collected information and succeed in project results reports. Since the thesis has been published on July 2012, results and images refer to two measurements period of acquisition for most the patients, even though the number of subjects undergoing THR and agreeing the program is increasing thus new patients will be expected to reach significant groups of study. Having examined anyway partial results, concerning outcomes collected through modeling and gait analysis tools, we can afford to deal a general framework on prior and posterior conditions of patients respecting the THR operation.

The BMD acquisition realizes a modeling tool for prior and posterior evaluations. It indeed results to be an important instrument of analysis for bone quality, from which taking advantage in order to ascertain effective functionality of injured bone and therefore secure an important decision, given that bone's features are strictly connected to implants prerogatives entirely chosen according to. Where porous bone dominates the femur shape, the BMD observation matches expectations of patients treated by cemented implant according to lower density level in average. Otherwise, cementless implants have been considered suited for a femur replacement where the prosthesis setting might be facilitated by bone strength, this last pointed out trough average higher BMD values in charts reported. As surgeons got decisions managing CT scan not elaborated prospects, notwithstanding the acquisition and elaborating protocol might be truly improved for this kind of tool, nowadays they can eagerly benefit from those data beforehand.

The main analysis outcome achieves the estate to be an important support trough objective scientific value. A significant future application might be the possibility to evaluate alterations in bone mass after one year of rehabilitation, when it is supposed to perform further CT scan. As the modification of load on the operated joint led critical issues or beneficial improvements in walk stride as well, hopefully the gait rite and BMD combination might be meaningful. Further it can be likely an instrument of control for all application based on aging-bone quality relation in hospital carried out The Strain test analysis represents the second part of modeling work accomplished. As already said, intraoperative periprosthetic fractures are becoming more common given the increased prevalence of revision total hip arthroplasty and increased use of cementless fixation. Actually risk factors include the use of minimally invasive techniques now commonly adopted by surgeons of the hospital as the use of press- fit cementless stems and technical errors at the time of the operation have been reported before the project setting up. Therefore, the simulation of doctors actions on the femur, in terms of solicitations applied to the bone, might warn the surgeon about certain criticism of the femur undergoing THR. Results point out critical zones of failure risk and objective index of failure identified by percentage values of FRI. The layout matches expectations of surgeons concerning usual zone of breaking risk as the protocol has been performed and improved with their experiences compared to. Future improvements will likely regard the force setting hypothesis in order to simulate also the hitting of the hammer acted by surgeons This part of the project will be powered by the new collaboration with MIT researcher recently involved in.

The analysis of gait has got possible thank to Grensas Hospital of Rehabilitation and physiotherapists involved in the project. Measurements acquisitions have been supervised by them while biomedical engineering students were collecting data. Tables, virtual patient paths and pressure maps have been collected in order to obtain a complete outcome regarding walk features of subjects' pre and post operation, and further one year later. This kind of work is useful for people involved in the rehabilitation follow up. They can assess gains, in terms of asymmetries disappearances for certain parameters between operated and healthy side for example, thus representing a sort of objective follow up of the patient along the project period of acquisition until one year after the surgery. Nonetheless, parameters were anyway collected and sorted by kind of implant, as similarly it has been done for modeling part of the project. The results, for selected parameters at least, do not evidence particular trends connected to prosthesis used by surgeons and might be expected an absence of meaningful link. What we observed was higher variability in "cemented" patients among the different acquisitions for single patients, revealed by wider intervals of confidences for the values drawn. Given that these are partial results, in a shortly future new data will be gained and therefore an overall complete prospect might clarify the situation, in order to evidence unequal normal walking recovery between two principal groups of study. Furthermore, a work section representing modeling and SEMG acquisition on certain thigh muscles has been reported in the thesis and will be likely deepened for future development once new CT scans and Kine pro acquisition will be available. Therefore the results, even though in partial form have been presented, give us the possibility to assess any gain for the single patients compared with initial conditions and during the process of rehabilitation. Indeed this part represents such an important tool of inquisition focused on muscles mass that however is a clear sign of wellness of the patient in the walk stride, it affords furthermore to point out 
correspondences in electrical paths strictly connected to. The database developed by the author has proved to be very useful to collect and report data. Final outcomes from each part of the project work are continuously stored in the program database, or rather have been reported trough the path that reaches the objects in hospital servers. As usually happened when a huge quantity of elements of study has to be stored, the project risks creating confusion and redundant information might be offered to final customers, in a meaningful way neither. Despite elaborations can be well realized and all the work accomplished in a correct way, actually tough an important role is covered by the manner trough which results are shown to. What the database offers are multiple layouts representing ordered views on project results managed by users of the department, and thankfully it has been already a useful tool of report for surgeons during meetings in hospital.

\section{Abbreviations}

AAOS: American Academy of Orthopaedic surgeons; BMAD: Bone Mineral Apparent Density; BMD: Bone Mineral Density; CAD: Computer-Aided Design; CEM: Cemented Implants; CHD: Congenital Hip Dislocation; COM: Center of Mass; CT: Computed Tomography; DB: Database; DBMS: Database Management System; DEXA: Dual Energy X ray Absorptiometry; DJD: Degenerative Joint Diseases; FEA: Finite Element Analysis; FEM: Finite Element Method; FRI: Fracture Risk Index; HU: Hounsfield Unit; ICD: International Classification of Diseases; JRF: Joint Reaction Force; LCP: Legg Calve Perthes disease; NJR: National Joint Registry; PMMA: Polymethylmethacrylate; ROI: Region of Interest; SDO: Scheda di Dimissione Ospedaliera (Ital); sEMG: Surface Electromyography; SENIAM: Surface ElectroMyography for the Non Invasive Assessment of Muscles; THA: Total Hip Arthroplasty; THR: Total hip Replacement; UNCEM: Cementless Implants.

\section{Acknowledgements}

This work was possible because of the support and the cooperation of many people. I would like to thank and express my appreciation to my reviewers: Prof. P. Gargiulo, Prof. M. Cesarelli and Prof. P. Bifulco. I thank the Landspitali-University Hospital of Iceland personnel and people involved in the project that made this work possible, in particular Gígja Magnúsdóttir, Grétar Halldórsson, Guðbjörg Kristín Ludvigsdóttir, Jan Tribel and Halldór Jónsson jr. I very much appreciate the support I received from Reykjavik University students during the study, for this I thank pröstur Pétursson and Benedikt Magnússon.

\section{Corresponding Author}

Gianluca Mario Izzo
University of Naples Federico II, Dept. of Biomedical, electronic and telecommunications engineering, Italy; and Dept. of Science, Education and Innovation, Landspitali University Hospital, Iceland; Dept. of Biomedical Engineering, University of Reykjavik, Iceland

Address: Via delle Vigne 57, 04023 Formia (LT) Italy

Phone: +39 3280503559

E-mail: gi.88@hotmail.it

\section{References}

[1] http://www.nlm.nih.gov/medlineplus.

[2] Adams M. F.,. Bayraktar H. H, Keaveny T. M., and Papadopoulos P.. Ultrascalable implicit finite element analyses in solid mechanics with over a half a billion degrees of freedom. In Proceedings of the 2004 ACM/IEEE conference on Supercomputing, Washington, DC, USA, 2004. IEEE Computer Society.

[3] A. M. A. American medical association. Mechanic of hip joint. 2012.

[4] Basmajian J. Muscles alive, their functions revealed by electromyography. Williams \& Wilkins, 1978.

[5] Behrens B., Nolte I., Wefstaedt, P.. StukenborgColsman C, and Bouguecha A.. Numerical investigations on the strain adaptive bone remodelling in the periprosthetic femur: Influence of the boundary conditions. BioMedical Engineering OnLine, 8:1-9, 2009.

[6] Bergmann G., Deuretzbacher G., Heller M., Graichen F., Rohlmann A., Strauss J., and. Duda G. N. Hip contact forces and gait patterns from routine a ctivities. Journal of Biomechanics, 3 4(7):859-871, 2001.

[7] Bilney B., Morris M., and. Webster K. Gaitrite electronic walkway technical reference (wi-0215) rev.i page 37 of 44 . Document WI-02-15 Filename: WI-02-15 Rev. I.

[8] Bilney B.,. Morris M, and Webster K.. Concurrent related validity of the gaitrite walkway system for quantification of the spatial and temporal parameters of gait. Gait and Posture 17 68:74, 2003.

[9] Blackburn J.,. Hodgskinson R, Currey J. D., and. Mason J. E. Mechanical properties of microcallus in human cancellous bone. J Orthop Res, 1 0(2):237-46, 1992.

[10] Boraiah S.,. Ragsdale M, Achor T., Zelicof, S. and Asprinio D. E.. Open reduction internal fixation and primary total hip arthroplasty of selected acetabular fractures. Journal of Orthopaedic Trauma, 23(4):243-248, 2009.

[11] Burr M., Choi S. W.,. Galehouse B, and. Yap C. Complete subdivision algorithms, ii: Isotopic 
Total hip replacement: structures modeling, gait analysis and report

European Journal Translational Myology - Basic Applied Myology 2012; 22 (1\&2): 69-121

meshing of singular algebric curves. CoRR, abs/1102.5463, 2011.

[12] Carter D. R., Bouxsein M. L., and Marcus R.. New approaches for interpreting projected bone densitometry data. Journal Of Bone and Mineral Research, 7(2):137_ ～45, 1992.

[13] Carter D. R. and Hayes W. C.. The compressive behavior of bone as a two- phase porous structure. The Journal of Bone and Joint Surgery, 59(7):954-962, 1977.

[14] Cavallo A., Cerbo M., Fella D., Jefferson T.and M. A. Hta report. Protsthesys for primary hip replacement in Italy, 2007.

[15] Cluett J.. Steps of a hip replacement. Santa Barbara CA USA, 2006. Health's Disease and Condition content.

[16] Cowin S. C.. Bone Mechanics Handbook. CRC Press, 2001.

[17] Currey J. D.. The design of mineralized hard tissues for their mechanical functions. Journal of Experimental Biology, 202(Pt 23):32853294, 1999.

[18] Currey J. D.. Bones: Structure and Mechanics.Princeton University Press, 2002.

[19] Dagenais S., Garbedian S., and Wai. E. K. Systematic review of the prevalence of radiographic primary hip osteoarthritis. Clinical Orthopaedics and Related Research, 467(3):623-637, 2009.

[20] Disselhorst C.-klug.European recommendations for surface electromyography. Signal Processing, 2010(February 4):8-11, 1999.

[21] Elble R., Moody C., Leffler K., and Sinha R.. The initiation of normal walking. Anderson Gait Analysis Laboratory, Rehabilitation Engineering Services, Princess Margaret Rose Orthopaedic Hospital, 2001.

[22] Gargiulo P., Helgason T., Reynisson P. J.,Helgason B., Kern H., Mayr W., Ingvarsson, P. and Carraro U.. Monitoring of muscle and bone recovery in spinal cord injury patients treatedwith electrical stimulation using threedimensional imaging and segmentation techniques: Methodological assessment. Artificial Organs, 35(3):275-281, 2011.

[23] Gargiulo P. and Reynisson P. J.. Phantom measurements. comparisons of instruments and measurements. 30 March 2010.

[24] Gargiulo P., Vatnsdal B., Ingvarsson P., Knutsdottir S., Gudmundottir V., Yngvason S., Kern H., Carraro U., and Helgason T. Computational methods to analyze tissue composition and structural changes in denervated muscle undergoing therapeutic electrical stimulation. pages 157- 161, 2010.
[25] Hailer N. P., Garellick G., and Karrholm J.. Uncemented and cemented primary total hip arthroplasty in the swedish hip arthroplasty register. Acta Orthopaedica, 81(1):34-41, 2010.

[26] Hansen U., Zioupos P., Simpson R., Currey J., and Hynd D.. The effect of strain rate on the mechanical properties of human cortical bone. Journal of Biomechanical Engineering, 130, 2008. [27] B.

[27] Helgason, E. Perilli, E. Schileo, F. Taddei, S. Brynjolfsson, and Viceconti M.. Mathematical relationships between bone density and mechanical properties: a literature review. Clinical Biomechanics, 23(2):135-146, 2008.

[28] Herman R. M., Grillner S., Stein P., and Stuart D.. Human solutions for locomotion. iii the initiation of gat. Neural control of locomotion, Plenum Press, New York, 65:7, 1976.

[29] Hodgskinson R. and Currey J. D..Effects of structural variation on Young's modulus of nonhuman cancellous bone. Proceedings of the Institution of Mechanical Engineers.Part $\mathrm{H}$, Journal of Engineering in Medicine, 204(1):43-52, 1990.

[30] Jäger M., Begg M. J. W., Ready J., Bittersohl, B., Millis M., Krauspe R., and Thornhill

T. S.. Primary total hip replacement in childhood, adolescence and young patients: Quality and outcome of clinical studies. Technol. Health Care, 16(3):195-214, Aug. 2008.

[31] Larkin A., Sheahan N., Gray L., O'Connor U., Dowling A., Vano E., Torbica PSalat, D., Schreiner A., Neofotistou V. Commissioning and quality assurance of dual energy $\mathrm{x}$-ray absorptiometry (dexa) systems. Rad. Prot. Dosim., 2008.

[32] Lütjering G. and Williams J.. Titanium. Engineering Materials and Processes. Springer, 2007.

[33] Mickelborougha J., Van der Linden M., Tallisc R., and Ennosd A.. Muscle activity during gait initiation in normal elderly people. Mov. Disord. 139:146, 1994.

[34] Noble P. C., Kamaric E.,. Sugano N, and. Matsubara M. Three-dimensional shape of the dysplastic femur: implications for thr. Clin Orthop Relat Res, pages 417:27- 40.41727, 2003.

[35] Oecd. Health at a glance: Europe 2010. 2010.

[36] Peacock M., Buckwalter K. A., Persohn S., Hangartner, T. Econs M., and Hui S.. Race and sex differences in bone mineral density and geometry at the femur. Bone, 45(2):218-225, 2009. 
Total hip replacement: structures modeling, gait analysis and report

European Journal Translational Myology - Basic Applied Myology 2012; 22 (1\&2): 69-121

[37] GargiuloP. Reynisson, P J, , Helgason B., Kern H., Mayr W., Ingvarsson P., Helgason T., and Carraro U.. Muscle, tendons and bone: structural changes during denervation and fes treatment. Neurol Res, 33, 2011.

[38] Reynisson P. J., Gargiulo P., and Helgason B.. Patellar bone strains in paraplegic patients undergoing functional electrical stimulation. Reykjavik University of Iceland Master Thesis, 2010.

[39] Ross S. M.. Introduction to Probability and Statistics for Engineers and Scientists, Fourth Edition. Academic Press, 2011.

[40] Snyder B. D., Piazza S., Edwards W. T., and Hayes W. C.. Role of trabecular morphology in the etiology of Age- Related vertebral fractures. Calcified tissue international, 53:S14-S22, 1993.

[41] A. Soininvaara, T. Miettinen, J. Hannu, S. Jurvelin, M. Alhava, and P. Kruger. Changes in bone mineral density of the proximal femur after total knee arthroplasty. The Journal of arthroplasty, $\quad$ 7(4):424-431, 2000.
[42] Sumner D. R. and Galante J. O.. Determinants of stress shielding: design versus materials versus interface. Clinical Orthopaedics and Related Research, (274):202- 212, 1992. [43] G. J. Tortora. Principles of Anatomy and Physiology, volume 9. Wiley international edition, 2009.

[44] Verhaar J. A. N.. Prognosis of total hip replacement: the importance of an implant register. Nederlands Tijdschrift Voor Geneeskunde, 151(35):1915-1917, 2007.

[45] Arm L. Voo, M., and Kleinberger. M. Stress fracture risk analysis of the human femur based on computational biomechanics. Johns Hopkins APL technical digest, 25(3):223-230, 2004.

[46] Walkway G. E.. Measuring function one step at a time. 2012.

[47] Weiss L.. Cell and tissue biology: a textbook of histology. Urban \& Schwarzenberg, 1988.

[48] Wolff J.. Das Gesetz der Transformation der Knochen. 1892. 


\section{A. GAIT RITE OVERALL SYNTHESIS}

\section{A.1 Statistic method for Gait Data}

In chapter 4 we faced out the method concerning gait rite analysis. Every patient overgoes the carpet at least 6 times per checkpoint. Thus, the need to synthesize the parameters considering all the tries is satisfied by subsequent statistical observations. The main hypothesis is to assume for every parameter the 6 or more measures as elements of a sample. Neverthless the sample is composed by few elements, we can definitely suppose our parameters distributed as a normal variable for which is possible to find out intervals of confidence. Suppose that $X_{1}, \ldots, X_{n}$ is a sample from a normal population having unknown mean $m$ (our final parameter) and known standard deviation $s$, calculated normally as Microsoft Excel allows to do.

It is proved that

$$
\bar{X}=\sum_{i=1}^{n} \frac{X_{i}}{n_{-}}
$$

$\mathrm{n}$ is the maximum likelihood estimator for $m$. However, we do not expect that the sample mean will exactly equal $m$, but rather that it will "be close". Hence, rather than a point estimate, it is sometimes more valuable to be able to specify one interval for which we have a certain degree of confidence that $m$ lies within. To obtain such an interval estimator, we make use of the probability distribution of the point estimator. In the foregoing, since the point estimator $\mathrm{X}$ is normal with mean $m$ and variance sigma squared divided $n$, it follows that

$$
\frac{\bar{X}-\mu}{\frac{\sigma}{n}}=\sqrt{n} \frac{\bar{X}-\mu}{\sigma}
$$

has a standard normal distribution. Now Z scores are measures of standard deviation. For example, if a tool returns a $\mathrm{Z}$ score of $+2,5$ it is interpreted as "+2.5 standard deviations away from the mean". P-values are probabilities. Both statistics are associated with the standard normal distribution. This distribution relates standard deviations with probabilities and allows significance and confidence to be attached to Z scores and p-values. the Z-value for the 95\% (actually $97.5 \%$ because $2.5 \%$ on each end is total 5\%) of the gaussian area, retrieved from a Z-table. Therefore

$$
P\left\{-1.96<\sqrt{n} \frac{X-\mu}{\sigma}<1.96\right\}=0.95
$$

Multiplying through by -1 yields the equivalent statement

$$
P\left\{X-1.96 \frac{\sigma}{\sqrt{n}}<\mu<X+1.96 \frac{\sigma}{\sqrt{n}}\right\}=0.95
$$

That is the $0,95 \%$ of the time $m$ will lie within 1 , 96(sigma square divided $n$ ) $n$ units of the sample average. If we

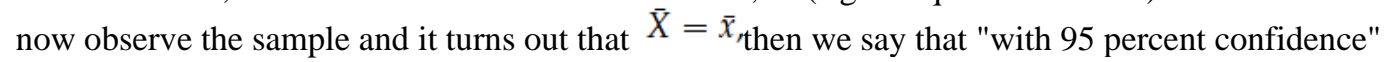

$$
x-1.96 \frac{\sigma}{\sqrt{n}}<\mu \sigma<x+1.96 \frac{\sigma}{\sqrt{n}}
$$

That is, "with 95 percent confidence" we assert that the mean lies within 1.96 (sigma square divided n) $\mathrm{n}$ of the observed sample mean. The interval is called 95 percent confidence interval estimate of $m$.

$$
\left(x-1.96 \frac{\sigma}{\sqrt{n}}, x+1.96 \frac{\sigma}{\sqrt{n}}\right)
$$


Total hip replacement: structures modeling, gait analysis and report

European Journal Translational Myology - Basic Applied Myology 2012; 22 (1\&2): 69-121

\section{A2 RESULTS}

\begin{tabular}{|c|c|c|c|c|c|c|c|c|c|c|c|c|c|}
\hline \multirow{2}{*}{$\begin{array}{l}\text { CEM } \\
\text { Age }\end{array}$} & \multirow{2}{*}{$\begin{array}{c}\text { PREOP } \\
\text { Side } \\
\end{array}$} & \multicolumn{3}{|c|}{ Step Time [s]-operated } & \multicolumn{3}{|c|}{ step Time [s] -healthy } & \multicolumn{3}{|c|}{ Cycle Time [s] - operated } & \multicolumn{3}{|c|}{ Cycle Time [s] -healthy } \\
\hline & & $95 \%-$ & Mean & $95 \%+$ & $95 \%-$ & Mean & $95 \%+$ & $95 \%-$ & Mean & $95 \%+$ & $95 \%-$ & Mean & $95 \%+$ \\
\hline 57 & L & 0,5430 & 0,5700 & 0,5970 & 0,5011 & 0,5220 & 0,5429 & 1,0503 & 1,0900 & 1,1297 & 1,0436 & 1,0940 & 1,1394 \\
\hline 65 & $\mathbf{R}$ & 0,5971 & 0,6080 & 0,6189 & 0,5760 & 0,5910 & 0,6060 & 1,1805 & 1,2050 & 1,2295 & 1,1780 & 1,1930 & 1,2180 \\
\hline 67 & $\mathbf{R}$ & 0,5086 & 0,5360 & 0,5634 & 0,4553 & 0,4980 & 0,5407 & 0,9613 & 1,0300 & 1,0987 & 1,0018 & 1,0560 & 1,0018 \\
\hline 70 & $R$ & 0,4429 & 0,4586 & 0,4742 & 0,4587 & 0,4700 & 0,4813 & 0,9038 & 0,9286 & 0,9533 & 0,9054 & 0,9300 & 0,9546 \\
\hline 72 & $R$ & 0,6841 & 0,7030 & 0,7219 & 0,6622 & 0,6880 & 0,7138 & 1,3632 & 1,3950 & 1,4268 & 1,3541 & 1,3890 & 1,4239 \\
\hline 74 & $\mathbf{R}$ & 0,5046 & 0,5217 & 0,5388 & 0,4888 & 0,5117 & 0,5345 & 0,9893 & 1,0283 & 1,0673 & 0,9904 & 1,0317 & 0,9904 \\
\hline 76 & $\mathbf{R}$ & 0,5674 & 0,5900 & 0,6126 & 0,5252 & 0,5529 & 0,5805 & 1,0951 & 1,1414 & 1,1878 & 1,0950 & 1,1443 & 1,1936 \\
\hline \multicolumn{2}{|l|}{ CEM } & \multicolumn{3}{|c|}{ Step Time [s]-operated } & \multicolumn{3}{|c|}{ step Time [s] -healthy } & \multicolumn{3}{|c|}{ Cycle Time [s] - operated } & \multicolumn{3}{|c|}{ Cycle Time [s] -healthy } \\
\hline Age & Side & $95 \%-$ & Mean & $95 \%+$ & $95 \%-$ & Mean & $95 \%+$ & $95 \%-$ & Mean & $95 \%+$ & $95 \%-$ & Mean & $95 \%+$ \\
\hline 57 & $L$ & 0,5270 & 0,5367 & 0,5464 & 0,5112 & 0,5200 & $0,528 \mathrm{~B}$ & 1,0436 & 1,0567 & 1,0697 & 1,0361 & 1,0517 & 1,0672 \\
\hline 65 & $R$ & 0,6376 & 0,6450 & 0,6524 & 0,6121 & 0,6238 & 0,6354 & 1,2572 & 1,2663 & 1,2753 & 1,2582 & 1,2713 & 1,2843 \\
\hline 65 & $L$ & 0,4382 & 0,4960 & 0,5038 & 0,4766 & 0,4880 & 0,4994 & 0,9689 & 0,9860 & 1,0031 & 0,9736 & 0,9900 & 1,0064 \\
\hline 67 & $\mathbf{R}$ & 0,5247 & 0,5320 & 0,5393 & 0,5393 & 0,5680 & 0,5967 & 1,0528 & 1,0960 & 1,1392 & 1,0904 & 1,1100 & 1,1296 \\
\hline 70 & $\mathbf{R}$ & 0,5392 & 0,5550 & 0,5708 & 0,5292 & 0,5450 & 0,5608 & 1,0664 & 1,0967 & 1,1269 & 1,0718 & 1,1000 & 1,1282 \\
\hline 74 & $R$ & 0,5053 & 0,5180 & 0,5307 & 0,4969 & 0,5080 & 0,5109 & 1,0123 & 1,0340 & 1,0557 & 1,0038 & 1,0260 & 1,0482 \\
\hline 76 & $R$ & 0,5605 & 0,5867 & 0,6128 & 0,5504 & 0,5700 & 0,5896 & 1,0597 & 1,1198 & 1,1799 & 1,1120 & 1,1533 & 1,1947 \\
\hline \multicolumn{2}{|l|}{ UNCEM } & \multicolumn{3}{|c|}{ Step Time [s]- operated } & \multicolumn{3}{|c|}{ Step Time [s] -healthy } & \multicolumn{3}{|c|}{ Cycle Time [s]- operated } & \multicolumn{3}{|c|}{ Cycle Time [s] -healthy } \\
\hline Age & Side & $95 \%-$ & Mean & $95 \%+$ & $95 \%-$ & Mean & $95 \%+$ & $95 \%-$ & Mean & $95 \%+$ & $95 \%-$ & Mean & $95 \%+$ \\
\hline 20 & $\mathbf{R}$ & 0,4627 & 0,4833 & 0,5040 & 0,4677 & 0,4783 & 0,4890 & 0,9360 & 0,9583 & 0,9806 & 0,9307 & 0,9633 & 0,9960 \\
\hline 44 & L & 0,5525 & 0,5717 & 0,5909 & 0,5549 & 0,5600 & 0,5651 & 1,1266 & 1,1383 & 1,1501 & 1,1027 & 1,1300 & 1,1573 \\
\hline 49 & $\mathbf{R}$ & 0,4992 & 0,5150 & 0,5308 & 0,4732 & 0,4900 & 0,5068 & 0,9719 & 1,0033 & 1,0348 & 0,9707 & 1,0033 & 1,0360 \\
\hline 51 & L & 0,5469 & 0,5650 & 0,5831 & 0,5118 & 0,5267 & 0,5416 & 1,0554 & 1,0883 & 1,1213 & 1,0601 & 1,0900 & 1,1199 \\
\hline 55 & $R$ & 0,5515 & 0,5614 & 0,5714 & 0,5746 & 0,5829 & 0,5911 & 1,1353 & 1,1471 & 1,1590 & 1,1279 & 1,1400 & 1,1521 \\
\hline 62 & L & 0,6053 & 0,6130 & 0,6307 & 0,5839 & 0,5970 & 0,6101 & 1,1909 & 1,2150 & 1,2391 & 1,1895 & 1,2160 & 1,2425 \\
\hline 67 & $R$ & 0,4905 & 0,5013 & 0,5120 & 0,4893 & 0,5025 & 0,5157 & 0,9817 & 1,0063 & 1,0308 & 0,9825 & 1,0050 & 1,0275 \\
\hline \multicolumn{2}{|l|}{ UNCEM } & \multicolumn{3}{|c|}{ Step Time [s] - operated } & \multicolumn{3}{|c|}{ Step Time [s] -healthy } & \multicolumn{3}{|c|}{ Cycle Time [s] - operated } & \multicolumn{3}{|c|}{ Cycle Time [s] -healthy } \\
\hline Age & Side & $95 \%-$ & Mean & $95 \%+$ & $95 \%-$ & Mean & $95 \%+$ & $95 \%-$ & Mean & $95 \%+$ & $95 \%-$ & Mean & $95 \%+$ \\
\hline 20 & $\mathbf{R}$ & 0,5125 & 0,5222 & 0,5319 & 0,5247 & 0,5333 & 0,5420 & 1,0351 & 1,0533 & 1,0715 & 1,0373 & 1,0533 & 1,0693 \\
\hline 44 & L & 0,5429 & 0,5660 & 0,5891 & 0,5568 & 0,5640 & 0,5712 & 1,1215 & 1,1360 & 1,1505 & 1,1051 & 1,1280 & 1,1509 \\
\hline 49 & $R$ & 0,5010 & 0,5129 & 0,5247 & 0,4986 & 0,5014 & 0,5042 & 1,0010 & 1,0157 & 1,0304 & 1,0037 & 1,0157 & 1,0277 \\
\hline 62 & $\mathrm{~L}$ & 0,5535 & 0,5600 & 0,5665 & 0,5742 & 0,5830 & 0,5918 & 1,1322 & 1,1440 & $1,155 B$ & 1,1302 & 1,1430 & 1,1558 \\
\hline 67 & $R$ & 0,5499 & 0,5613 & 0,5726 & 0,5552 & $0,568 B$ & 0,5823 & 1,1112 & 1,1313 & 1,1513 & 1,1083 & 1,1350 & 1,1617 \\
\hline
\end{tabular}

Table 7 Temporal parameters schedule
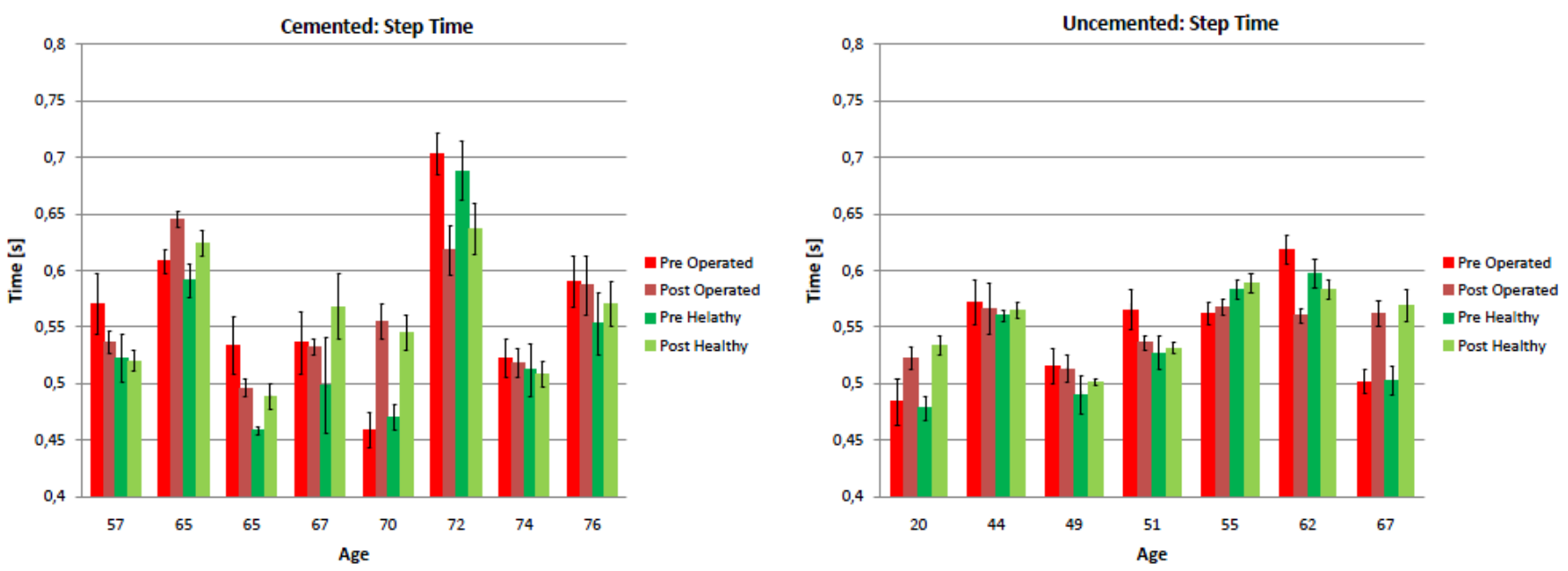

Fig 54 Step time results 
Total hip replacement: structures modeling, gait analysis and report

European Journal Translational Myology - Basic Applied Myology 2012; 22 (1\&2): 69-121
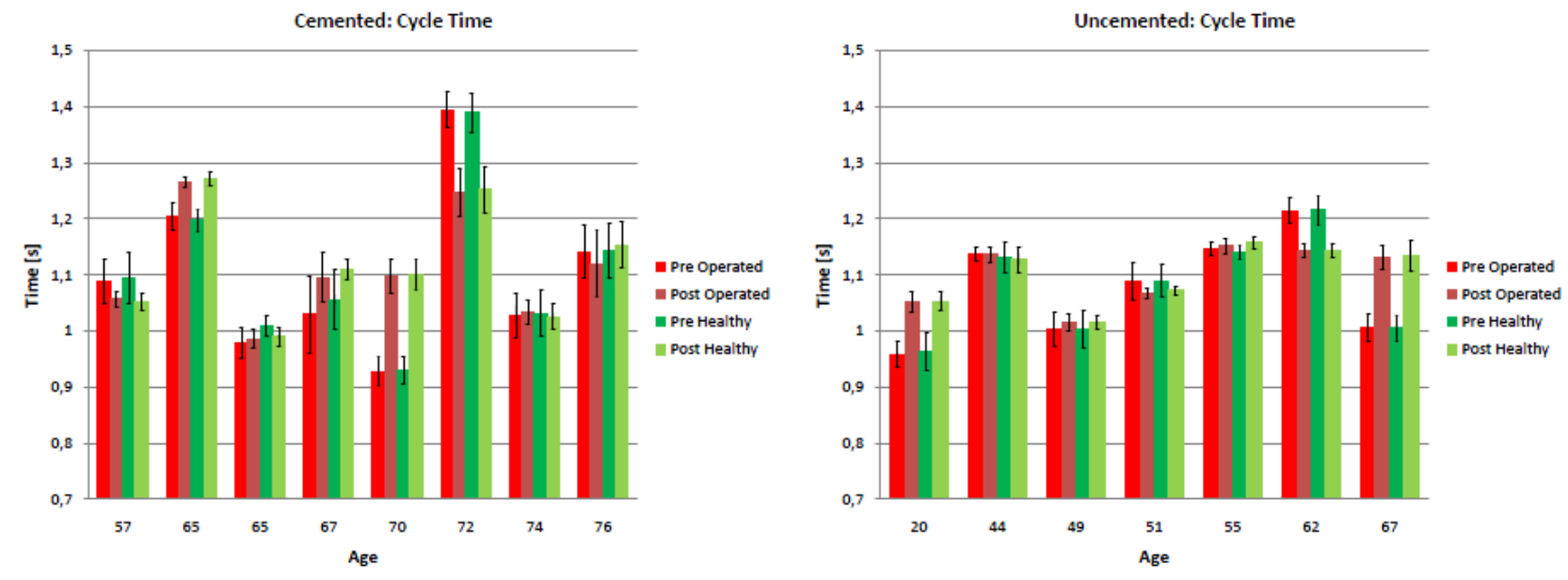

Fi 55 Cycle time results
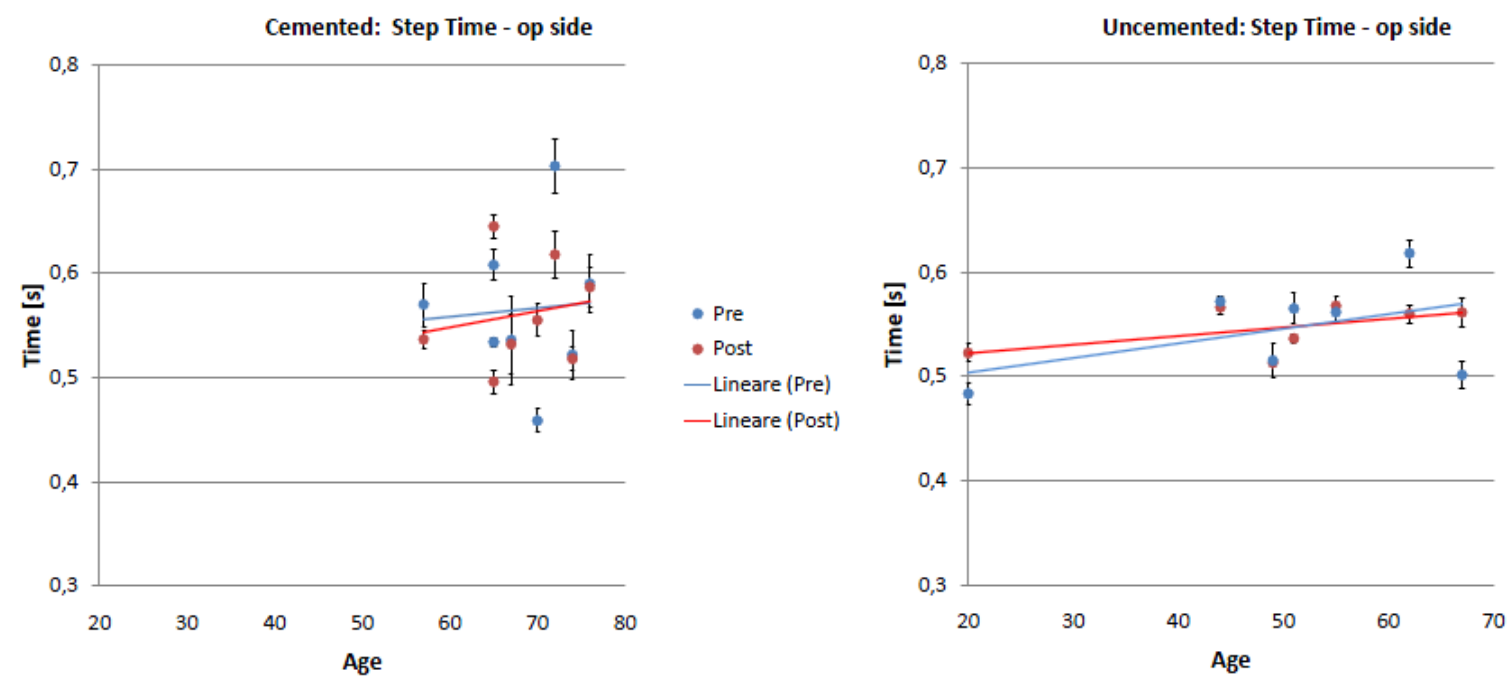

Fig 56 Scatter view for step time
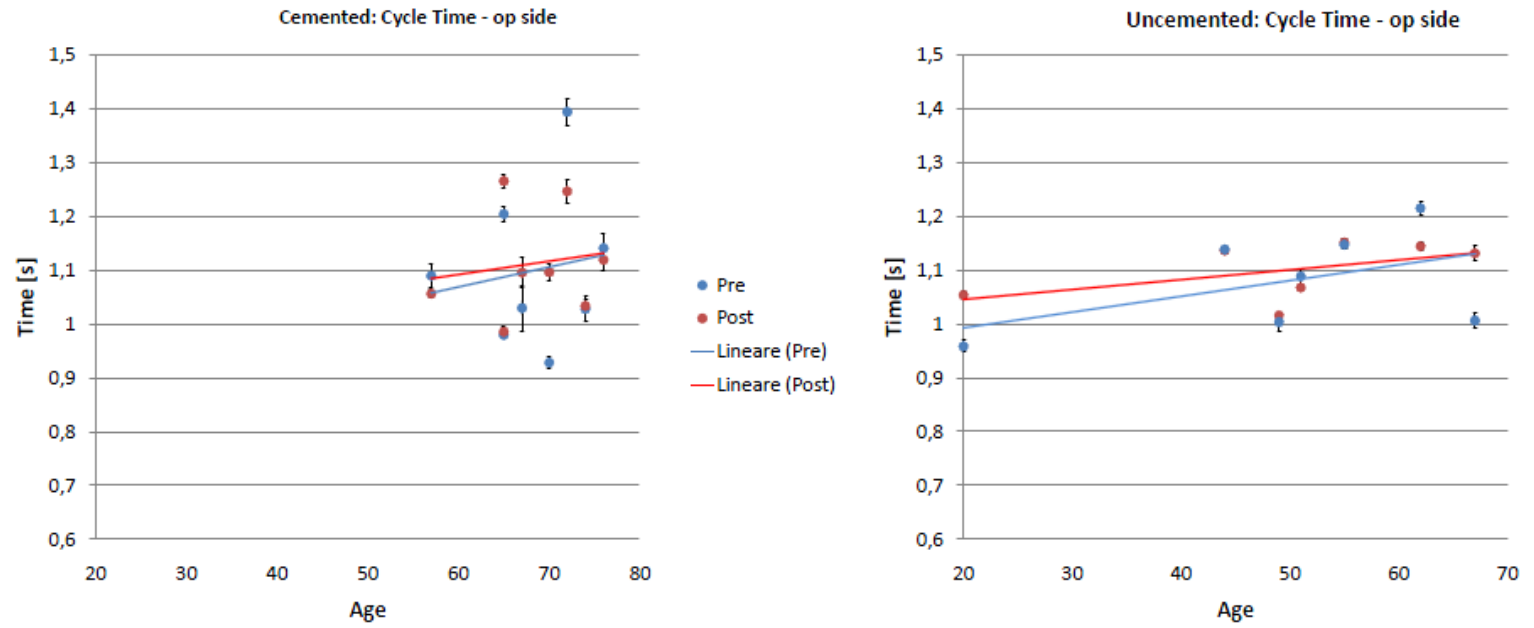

Fig 57 Scatter view for cycle time 
Total hip replacement: structures modeling, gait analysis and report

European Journal Translational Myology - Basic Applied Myology 2012; 22 (1\&2): 69-121

\begin{tabular}{|c|c|c|c|c|c|c|c|c|c|c|c|c|c|}
\hline \multirow{2}{*}{$\begin{array}{l}\text { CEM } \\
\text { Age }\end{array}$} & \multirow{2}{*}{$\begin{array}{c}\text { PREOPP } \\
\text { Side }\end{array}$} & \multicolumn{3}{|c|}{ Step length $[\mathrm{cm}]$ - operated } & \multicolumn{3}{|c|}{ Step length [cm] -healthy } & \multicolumn{3}{|c|}{ Stride length $[\mathrm{cm}]$ - operated } & \multicolumn{3}{|c|}{ Stride length $[\mathrm{cm}]$-healthy } \\
\hline & & $95 \%-$ & Mean & $95 \%+$ & $95 \%-$ & Mean & $95 \%+$ & $95 \%-$ & Mean & $95 \%+$ & $95 \%$ - & Mean & $95 \%+$ \\
\hline 57 & $\mathrm{~L}$ & 51,71351 & 54,362 & 57,01049 & 55,60704 & 57,54 & 59,47296 & 108,3201 & 112,114 & 115,9079 & 107,7382 & 112,442 & 117,1458 \\
\hline 65 & $R$ & 45,64036 & 46,53 & 47,41964 & 47,99867 & 49,143 & 50,28733 & 94,51265 & & 97,73335 & 94,45064 & 96,158 & \\
\hline 65 & $L$ & 48,46418 & 55,202 & 61,93982 & 47,93925 & 51,368 & 54,79675 & 97,0558 & 106,938 & 116,3202 & 103,7563 & 110,218 & 116,6797 \\
\hline 67 & $\mathbf{R}$ & 65,84858 & 67,502 & 69,15542 & 54,23386 & 58,24 & 62,24614 & 121,0624 & 125,942 & 130,8216 & 123,4319 & 128,07 & 132,7081 \\
\hline 70 & $\mathbf{R}$ & 3288 & 62,10429 & 62,87977 & 58,30395 & 59,07143 & 59,8389 & 119,8351 & 121,3271 & 122,8191 & 119,8184 & 120,9186 & 122,0 \\
\hline 72 & $R$ & 38,16533 & 39,724 & 41,28267 & 34,54495 & & 39,71705 & 73,96703 & 77,454 & & 73,14568 & 76,822 & \\
\hline 74 & $\mathrm{R}$ & 61,06861 & 62,84167 & 64,61473 & 62,26087 & 64,25333 & 66,24579 & 124,7961 & & 130,9239 & 123,3869 & 127,225 & \\
\hline 76 & $R$ & 45,20685 & 48,00143 & \begin{tabular}{|l|}
50,796 \\
\end{tabular} & 46,01066 & 48,43714 & 50,86362 & 91,80596 & 96,85286 & 101,8998 & 91,44605 & 96,67 & 101,8939 \\
\hline \multicolumn{2}{|l|}{ CEM } & \multicolumn{3}{|c|}{ Step length $[\mathrm{cm}]$ - operated } & \multicolumn{3}{|c|}{ Step length $[\mathrm{cm}]$-healthy } & \multicolumn{3}{|c|}{ Stride length $[\mathrm{cm}]$ - operated } & \multicolumn{3}{|c|}{ Stride length $[\mathrm{cm}]$-healthy } \\
\hline Age & Side & $95 \%-$ & Mean & $95 \%+$ & $95 \%-$ & Mean & $95 \%+$ & $95 \%-$ & Mean & $95 \%+$ & 95\%- & \begin{tabular}{|l|} 
Mean \\
\end{tabular} & $95 \%+$ \\
\hline 57 & L & 53,63052 & 54,79333 & 55,95615 & 48,58776 & 49,29333 & 49,99891 & 102,5864 & 104,2167 & 105,8469 & 102,7101 & 104,3533 & 105,9965 \\
\hline 65 & $R$ & 51,53363 & \begin{tabular}{|l|}
52,41875 \\
\end{tabular} & 53,30387 & 46,81051 & 47,6 & 48,38949 & 99,40291 & 100,4538 & 101,5046 & 98,92975 & 100,2288 & 101,5278 \\
\hline 65 & L & 66 , & & 68,78 & 67, & 12 & 163 & & & 839 & &, 444 & \\
\hline 67 & $R$ & 70 , & & 71,69165 & & & & & & & & & \\
\hline 70 & $R$ & 48,43506 &, 44 & 52,44494 & 42,19445 & 19 & 48,18555 & 90,96238 & 96,055 & 101,1476 & 91,67267 & 96,185 & 100,6 \\
\hline 72 & $R$ & 51,56097 & 54 & 55,51903 & 50,79807 & 2,182 & 593 & 102,6077 & 04 & 109,4723 & 102,5668 & 105,751 & 108 \\
\hline 74 & $R$ & 59,61059 & 61,168 & \begin{tabular}{|l|}
62,72541 \\
\end{tabular} & 63,88983 & 65,03 & \begin{tabular}{|l|}
66,17017 \\
\end{tabular} & 124,8565 & 126,992 & 129,1275 & 124,0303 & 126,725 & 129,4 \\
\hline 76 & $R$ & 46,66802 & 49,75167 & 52,83532 & 45,63898 & 47,92833 & 50,21769 & 93,06982 & 98,07333 & 103,0768 & 92,20031 & 97,52667 & 102,8525 \\
\hline \multicolumn{2}{|l|}{ UNCEM } & \multicolumn{3}{|c|}{ Step Length $[\mathrm{cm}]$ - operated } & \multicolumn{3}{|c|}{ Step Length $[\mathrm{cm}]$ - healthy } & \multicolumn{3}{|c|}{ Stride length $[\mathrm{cm}]$ - operated } & \multicolumn{3}{|c|}{ Stride length $[\mathrm{cm}]$-healthy } \\
\hline Age & Side & $95 \%-$ & Mean & $95 \%+$ & $95 \%-$ & Mean & $95 \%+$ & $95 \%-$ & \begin{tabular}{|l|} 
Mean \\
\end{tabular} & $95 \%+$ & \begin{tabular}{|l|}
$95 \%-$ \\
\end{tabular} & \begin{tabular}{|l|} 
Mean \\
\end{tabular} & $95 \%+$ \\
\hline 20 & $R$ & 71,83051 & 72,69 & 73,49949 & 69,0839 & 70,855 & 72,67161 & 140,868 & 143,9417 & 147,0154 & 140,7664 & 143,7033 & 146,6402 \\
\hline 44 & $L$ & 56,72876 & 57,89625 & 59,06374 & 53,45905 & 55,1975 & 56,93595 & 110,8738 & 113,7575 & 116,6412 & 110,3868 & 113,3338 & 116,2 \\
\hline 49 & $R$ & & 71,33333 & 73,39537 & & & & & & & & 137,8967 & \\
\hline 51 & L & 64,53568 & 66,74 & 68,94432 & 63,36845 & 64,69333 & 66,01 & 128,4276 & 233 & 619 & 128,3977 & 3967 & \\
\hline 55 & $R$ & 57,31696 & 58,38571 & 59,45447 & 48,26915 & 49,10429 & 49,93942 & 105,6812 & 108,0871 & 110,4931 & 106,1677 & 107,7286 & 109,2894 \\
\hline 62 & $\mathrm{~L}$ & 46,99434 & 48,18 & 49,36566 & 49,49253 & 51,034 & 52,57547 & 96,98512 & 99,933 & 102,8809 & 97,6172 & 99,903 & 102,1888 \\
\hline 67 & $R$ & 54,55956 & 56,1 & 57,64044 & 51,73141 & 53,7175 & 55,70359 & 106,5686 & 110,2913 & 114,0139 & 106,9439 & 110,1888 & 113,4336 \\
\hline \multicolumn{2}{|l|}{ CEM } & \multicolumn{3}{|c|}{ Step Length $[\mathrm{cm}]$ - operated } & \multicolumn{3}{|c|}{ Step length $[\mathrm{cm}]$-healthy } & \multicolumn{3}{|c|}{ Stride length $[\mathrm{cm}]$ - operated } & \multicolumn{3}{|c|}{ Stride length $[\mathrm{cm}]$-healthy } \\
\hline Age & Side & $95 \%-$ & Mean & $95 \%+$ & $95 \%-$ & Mean & $95 \%+$ & $95 \%-$ & Mean & $95 \%+$ & $95 \%-$ & \begin{tabular}{|l|} 
Mean \\
\end{tabular} & $95 \%+$ \\
\hline 20 & $\mathrm{R}$ & 64,78512 & 66,72556 & 68,66599 & 52,72313 & 54,17556 & 55,62798 & 117,3137 & 120,9344 & 124,5552 & 118,3238 & 121,31 & 124,2962 \\
\hline 44 & L & 54,0936 & 55,94333 & 57,79307 & 48,20717 & 50,32 & 52,43283 & 102,6158 & 106,715 & 110,8142 & 103,4611 & 106,7233 & 109,9856 \\
\hline 49 & $R$ & 69,37801 & 70,21143 & 71,04485 & 55,59272 & 58,09571 & 60,59871 & 125,869 & 128,8757 & 131,8824 & 124,8342 & 128,1229 & 131,4116 \\
\hline 51 & $L$ & 68,67049 & 69,65875 & 70,64701 & 69,18664 & 70,175 & 71,16336 & 138,0232 & 139,8625 & 141,7018 & 138,2034 & 139,9788 & 141,7541 \\
\hline 55 & $R$ & 53,56998 & 54,845 & 56,12002 & 49,9326 & 50,82625 & 51,7199 & 104,5329 & 106,2313 & 107,9296 & 103,6409 & 105,395 & 107,1491 \\
\hline 62 & $L$ & 52,60094 & 53,643 & 54,68506 & 55,83841 & 56,593 & 57,34759 & 109,8878 & 111,296 & 112,7042 & 109,1744 & 110,508 & 111,8416 \\
\hline 67 & $\mathbf{R}$ & 49,02477 & 50,24375 & 51,46273 & 49,03059 & 50,325 & 51,61941 & 98,28796 & 100,6263 & 102,9645 & \begin{tabular}{|l|}
98,95389 \\
\end{tabular} & 101,2288 & 103,5036 \\
\hline
\end{tabular}

Table 8 Spatial parameters schedule
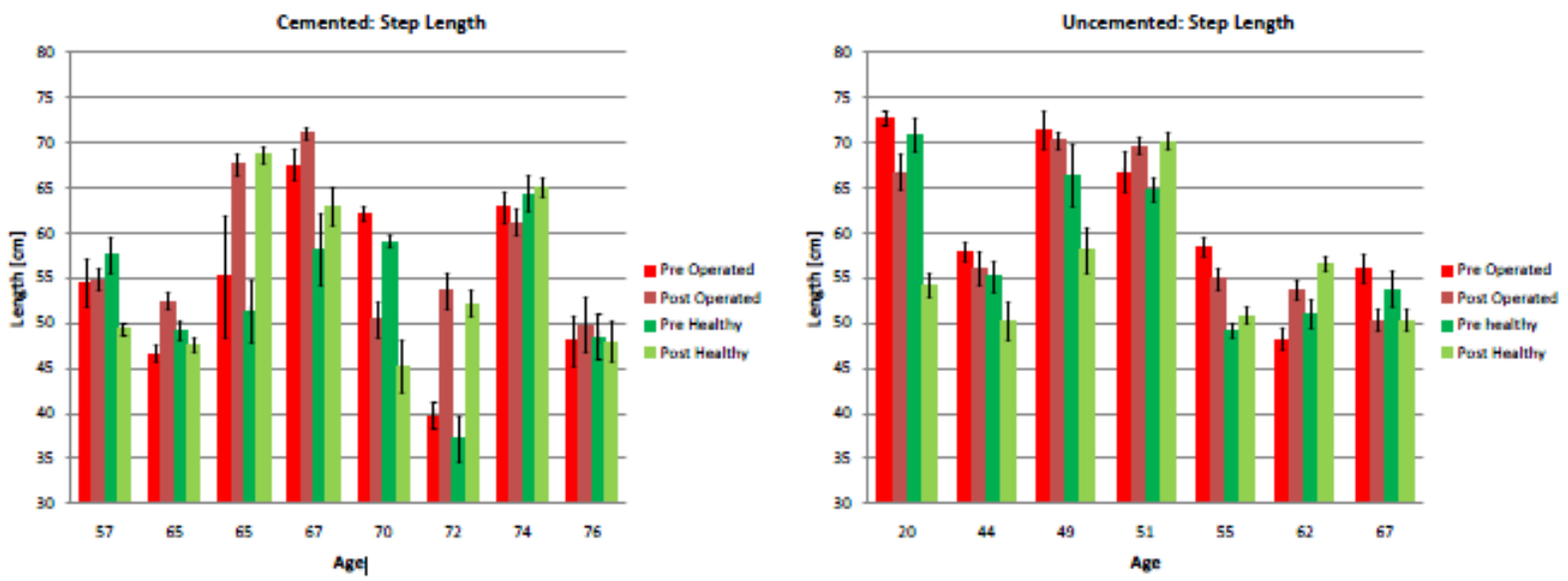

Fig 58 Step length 

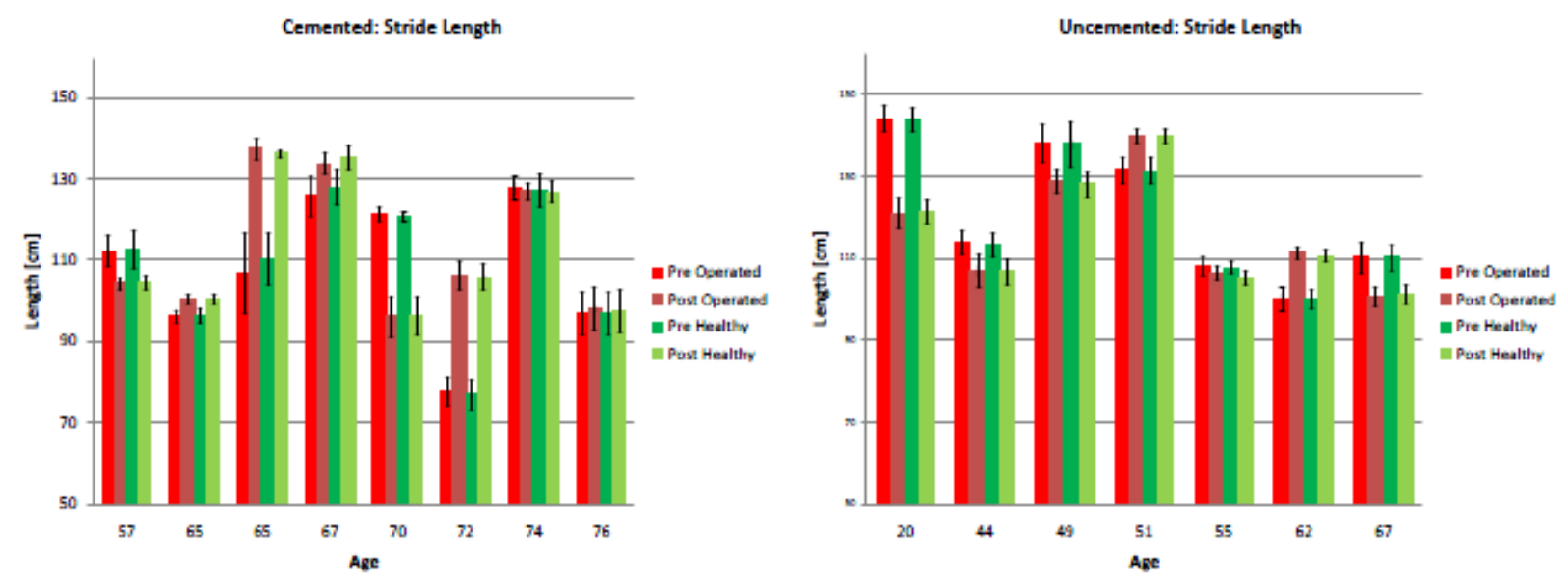

Fig 59 Stride length
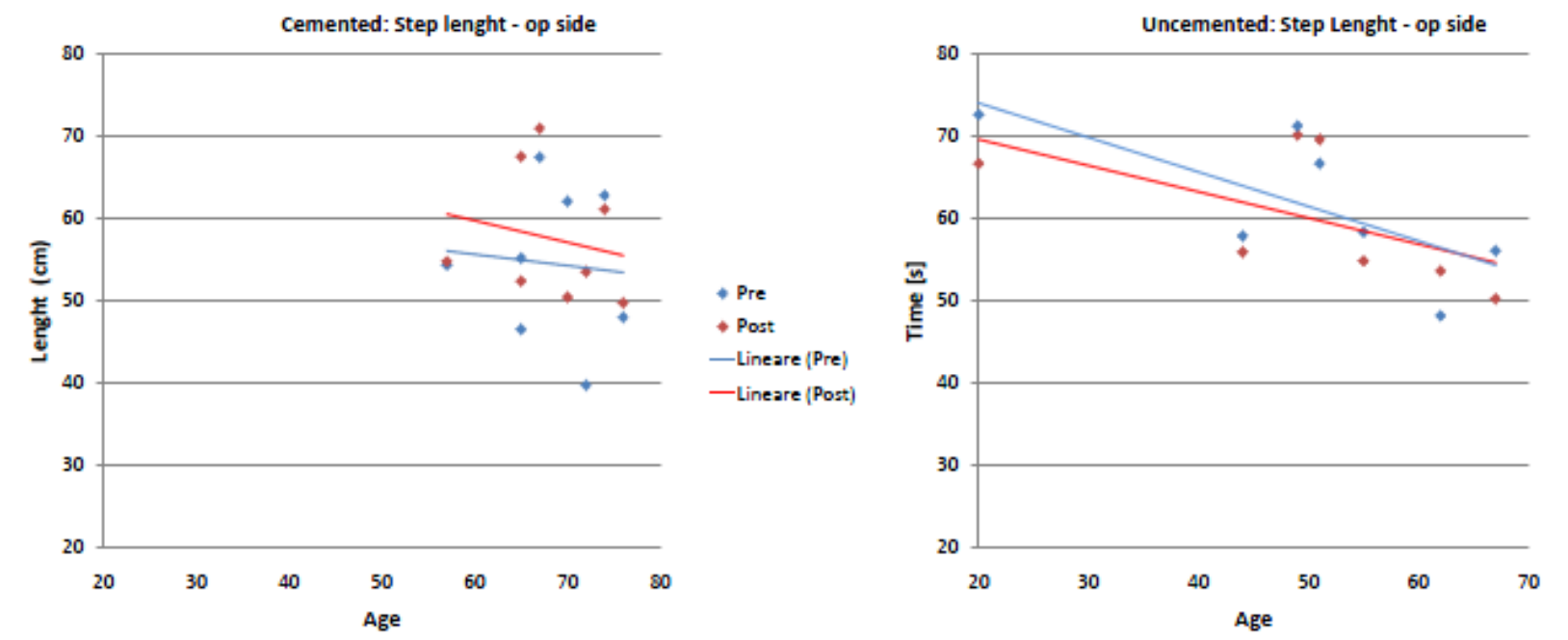

Fig 60 Scatter view for stride length

\section{Curriculum Vitae}

\section{Contact info}

Name: Gianluca Mario Izzo

Address: Via delle Vigne 57, 04023 Formia (LT) Italy

Phone: +39 3280503559; E-mail: gi.88@hotmail.it

\section{Main Career events:}

27-May 1988 Born in Pompei (Naples)

July 2006 Qualified at the Scientific Institute “L.B. Alberti” Minturno, Italy

2006 - 2009 Graduated at the University of Naples "Federico II” Bsc. Degree in Biomedical engineering

December 2009Bachelor Thesis in "Automatic analysis of Fingerprints and detection systems"

2010 - 2012 Graduated with maximum honour at the University of Naples "Federico II" Master in Biomedical engineering, Bioelectronic curriculum.

July 2012 Master thesis at Landspitalin, National hospital of Iceland and Reykjavik University of Iceland., named "Support for total hip replacement surgery: Structures modelling, Gait Data Analysis and Report system"

October to December 2012 New generation radar system specialist at CNIT of Naples 